\title{
Intermolecular Trifluoromethyl-Hydrazination of Alkenes Enabled by Organic Photo-Redox Catalysis
}

\author{
Peng Wang, Songsong Zhu, Dengfu Lu* and Yuefa Gong* \\ School of Chemistry and Chemical Engineering, Huazhong University of Science and Technology, \\ 1037 Luoyu Rd., Wuhan, Hubei, 430074, China. \\ E-mails: dlu@hust.edu.cn; gongyf@hust.edu.cn
}

\section{Table of Content}

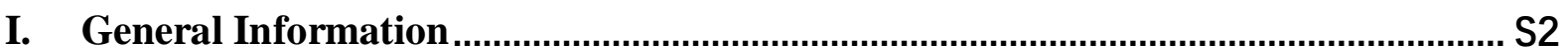

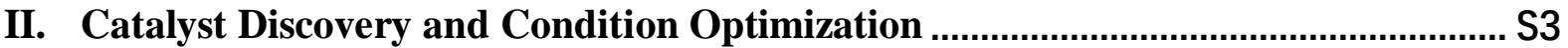

III. Trifluoromethyl-Hydrazination of Different Alkenes.......................................... S5

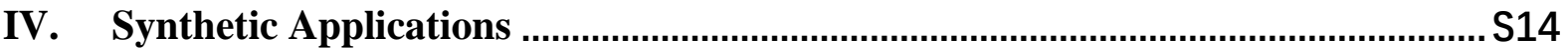

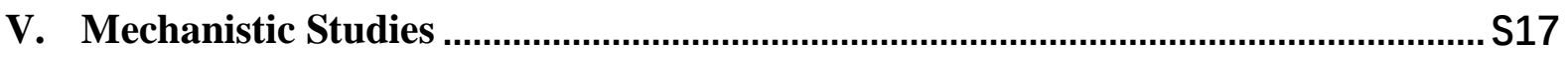

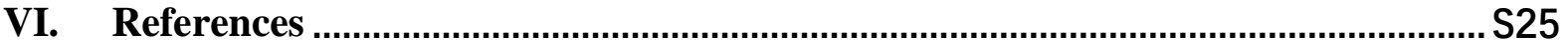

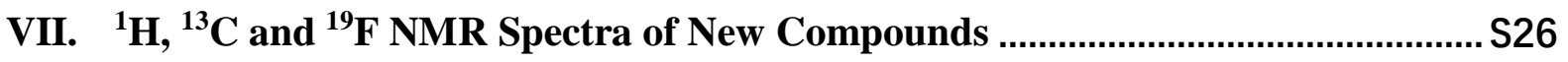




\section{General Information}

General procedures. $\mathrm{CF}_{3} \mathrm{SO}_{2} \mathrm{Na}$, di-tert-butyl azodicarboxylate and $\mathrm{Mes} \mathrm{AcrMe}^{+} \mathrm{ClO}_{4}{ }^{-}$were stored and weighed in a glovebox. Solvents were dried and freshly distilled before use. Reactions were monitored by thin-layer chromatography (TLC) using silica gel 60 F-254 plates. TLC plates were normally visualized by UV irradiation ( $254 \mathrm{~nm}$ or $365 \mathrm{~nm}$ ), stained with basic $\mathrm{KMnO}_{4}$ or phosphomolybdic acid. Flash chromatography was performed using silica gel 60 (200-300 mesh).

Instrumentation. Proton nuclear magnetic resonance ( ${ }^{1} \mathrm{H}$ NMR) spectra and proton-decoupled carbon nuclear magnetic resonance $\left({ }^{13} \mathrm{C}\left\{{ }^{1} \mathrm{H}\right\}\right.$ NMR) spectra were recorded on Bruker Ascend $400 \mathrm{MHz}$ or $600 \mathrm{MHz}$. Chemical shifts for protons are reported in parts per million downfield from tetramethylsilane and are referenced to the NMR solvent residual peak $\left(\mathrm{D}_{2} \mathrm{O}: \delta 4.79\right.$, $\left.\mathrm{CD}_{3} \mathrm{OD}: \delta 3.31\right)$. Chemical shifts for carbons are reported in parts per million downfield from tetramethylsilane and are referenced to the carbon resonances of the NMR solvent $\left(\mathrm{CD}_{3} \mathrm{OD}: \delta\right.$ 49.0). Data are represented as follows: chemical shift, multiplicity $(\mathrm{s}=\operatorname{singlet}, \mathrm{d}=\operatorname{doublet}, \mathrm{t}=$ triplet, $\mathrm{q}=$ quartet, $\mathrm{m}=$ multiplet, $\mathrm{br}=$ broad), coupling constants in Hertz $(\mathrm{Hz})$, and integration. IR spectra were recorded on a Bruker FT-IR spectrometer. HRMS was measured on a TOF-Q mass spectrometer equipped with an ESI source. High-performance liquid chromatography (HPLC) analysis was recorded on Shimadzu CBM-10A with a commercial Inertsil $4.6 \times 250$ mm column.

\section{Abbreviations:}

LED-light-emitting diode; TLC-thin layer chromatography; PE-Petroleum Ethers; THFtetrahydrofuran; DMSO-dimethyl sulfoxide; AIBN-Azobisisobutyronitrile; PTFEPolytetrafluoroethylene 


\section{Catalyst Discovery and Condition Optimization}

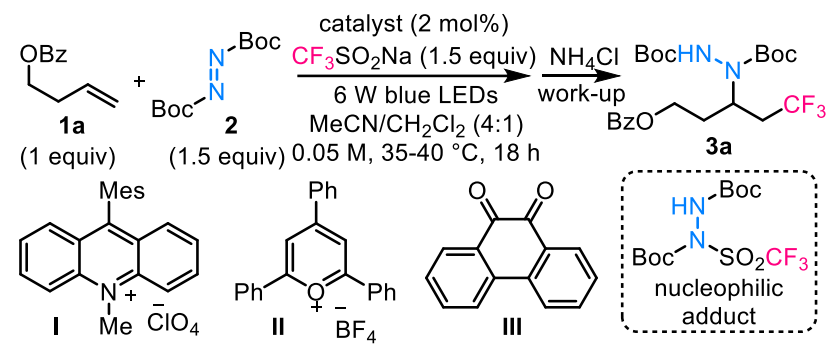

\begin{tabular}{|c|c|c|c|c|}
\hline entry & catalyst & variation from standard conditions & conv. $(\%)^{b}$ & Yield $(\%)^{c}$ \\
\hline 1 & I & none & $>95$ & 85 \\
\hline 2 & $\mathbf{I}$ & AcOH ( 1 equiv.) added & 89 & $69^{d}$ \\
\hline 3 & II & none & 82 & 35 \\
\hline 4 & III & none & 95 & 79 \\
\hline 5 & $\mathrm{Na}_{2}$-Eosin $\mathrm{Y}$ & none & 90 & 60 \\
\hline 6 & none & none & $<5$ & $<5$ \\
\hline 7 & $\mathbf{I}$ & performed in darkness & $<5$ & $<5$ \\
\hline 8 & $\mathbf{I}$ & in pure $\mathrm{CH}_{2} \mathrm{Cl}_{2}$ & $>95$ & 64 \\
\hline 9 & $\mathbf{I}$ & in pure $\mathrm{MeCN}$ & $>95$ & 80 \\
\hline 10 & $\mathbf{I}$ & $2 \& \mathrm{CF}_{3} \mathrm{SO}_{2} \mathrm{Na}$ (2 equiv) & $>95$ & $91(88)^{e}$ \\
\hline
\end{tabular}

${ }^{a}$ Unless otherwise stated, the reaction was performed under $\mathrm{N}_{2}$ with $1 \mathrm{a}(35 \mu \mathrm{L}, 0.2 \mathrm{mmol}), \mathrm{CF}_{3} \mathrm{SO}_{2} \mathrm{Na}(47 \mathrm{mg}, 0.3$ $\mathrm{mmol}), 2(69 \mathrm{mg}, 0.3 \mathrm{mmol})$ and a corresponding catalyst $(0.004 \mathrm{mmol})$ in $\mathrm{CH}_{3} \mathrm{CN} \mathrm{CH}_{2} \mathrm{Cl}_{2}(4: 1,4 \mathrm{~mL})$ at 35 to $40{ }^{\circ} \mathrm{C}$ under the irradiation of $6 \mathrm{~W}$ blue LEDs. ${ }^{b}$ The conversion of 1a was determined by HPLC with an internal standard. ${ }^{c}$ The yield of 3a was also measured by HPLC. ${ }^{d}$ A nucleophilic adduct was observed. ${ }^{e}$ Isolated yield

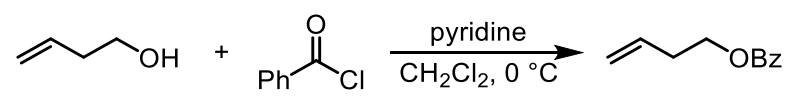

Compound $\mathbf{1 a}$ is a known compound and it was prepared according to a literature procedure. ${ }^{[1]}$ Di-tert-butyl azodicarboxylate $\mathbf{2}$ is commercially available and it was used as received.

General Procedures for catalyst discovery: To a sealable 2-dram vial equipped with a stir bar were added di-tert-butyl azodicarboxylate 2 (69 mg, $0.3 \mathrm{mmol}), \mathrm{CF}_{3} \mathrm{SO}_{2} \mathrm{Na}(47 \mathrm{mg}, 0.3$ mmol) and a corresponding photo-sensitizer $(0.004 \mathrm{mmol})$ in a glovebox. After the vial was taken out of the glovebox, anhydrous $\mathrm{CH}_{2} \mathrm{Cl}_{2}(0.8 \mathrm{~mL}), \mathrm{CH}_{3} \mathrm{CN}(3.2 \mathrm{~mL})$ and alkene 1a were added successively through syringes under $\mathrm{N}_{2}$. The reaction was then stirred under the irradiation of blue light (6 W LEDs. Note: the heat was genrated by the LED bulbs, and the photoreactor was maintained at between 35 to $40{ }^{\circ} \mathrm{C}$ by circulating water cooling). The reaction 
process was monitored with TLC. Upon the completion of reaction, the resulted mixture was quenched with saturated $\mathrm{NH}_{4} \mathrm{Cl}$ (aq., $2 \mathrm{~mL}$ ), extratced with EtOAc $(2 \mathrm{~mL} \times 3)$, dried over anhydrous $\mathrm{Na}_{2} \mathrm{SO}_{4}$ and concentrated in vacuo. The residue was redissolved in hexanes/ ${ }^{i} \mathrm{PrOH}$ (5:1, $20 \mathrm{~mL})$ together with dimethyl phthalate as an internal standard. After the insoluables were removed by a Nylon 66 synringe filter, the filtrate was subjected for HPLC analysis to determine the conversion (1a) and the yield (3a).

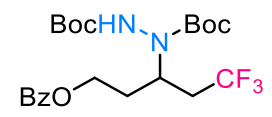

\section{Di-tert-butyl 1-(5-(benzoyloxy)-1,1,1-trifluoropentan-3-yl)hydrazine-1,2-dicarboxylate}

(3a): Pure 3a was isolated through a silica gel flash column (hexanes:EtOAc from 20:1 to 10:1) as colorless oil (84 mg, 88\% yield). ${ }^{1} \mathrm{H}$ NMR (600 MHz, DMSO- $\left.d_{6}, 343 \mathrm{~K}\right) \delta 8.93-8.19$ (brs, 1H), $8.20-7.87(\mathrm{~m}, 2 \mathrm{H}), 7.64(\mathrm{t}, J=7.5 \mathrm{~Hz}, 1 \mathrm{H}), 7.52(\mathrm{t}, J=7.7 \mathrm{~Hz}, 2 \mathrm{H}), 4.81-4.57$ (brs, 1H), $4.57-4.37$ (brs, 1H), $4.37-4.20$ (brs, 1H), $2.75-2.56(\mathrm{~m}, 1 \mathrm{H}), 2.46-2.30$ (m, 1H), $2.11-1.96(\mathrm{~m}, 1 \mathrm{H}), 1.96-1.79(\mathrm{~m}, 1 \mathrm{H}), 1.45(\mathrm{~s}, 9 \mathrm{H}), 1.41-1.28(\mathrm{~m}, 9 \mathrm{H}) ;{ }^{19} \mathrm{~F}$ NMR $(565$ MHz, DMSO- $\left.d_{6}, 343 \mathrm{~K}\right) \delta-58.00--71.18(\mathrm{~m}) ;{ }^{13} \mathrm{C}\left\{{ }^{1} \mathrm{H}\right\} \mathrm{NMR}\left(151 \mathrm{MHz}\right.$, DMSO- $\left.d_{6}, 343 \mathrm{~K}\right) \delta$ 166.1, 156.3, 154.0, 133.5, 130.6, 129.4, 129.0, 127.0 (q, $J=277.0 \mathrm{~Hz}), 80.8,80.2,62.0,50.1$, 48.6, $36.2(\mathrm{q}, J=24.1 \mathrm{~Hz}), 31.6,28.4,28.1$; IR $v_{\max }\left(\right.$ neat)/(cm ${ }^{-1}: 3313,2979,1713,1453,1376$, 1271, 1149, 712; HRMS (ESI, m/z): calcd for $\left.\mathrm{C}_{22} \mathrm{H}_{31} \mathrm{~F}_{3} \mathrm{~N}_{2} \mathrm{NaO}_{6}+\mathrm{M}+\mathrm{Na}\right]^{+}$499.2026, found 499.2022 .

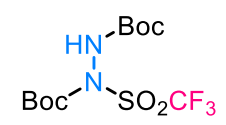

Di-tert-butyl 1-((trifluoromethyl)sulfonyl)hydrazine-1,2-dicarboxylate (9): compound 9 was isolated from the reaction described in entry 2 as a side product (white solid, m.p. 95.5 $\left.95.6{ }^{\circ} \mathrm{C}\right) ;{ }^{1} \mathrm{H}$ NMR $\left(400 \mathrm{MHz}, \mathrm{CDCl}_{3}\right) \delta 6.91(\mathrm{~s}, 1 \mathrm{H}), 1.48(\mathrm{~s}, 9 \mathrm{H}), 1.42(\mathrm{~s}, 9 \mathrm{H}) ;{ }^{19} \mathrm{~F}$ NMR $(376$ $\left.\mathrm{MHz}, \mathrm{CDCl}_{3}\right) \delta-72.21,-72.49$ (atropisomer); ${ }^{13} \mathrm{C}\left\{{ }^{1} \mathrm{H}\right\} \mathrm{NMR}\left(101 \mathrm{MHz}, \mathrm{CDCl}_{3}\right) \delta 153.1,148.3$, $119.3(\mathrm{q}, J=324.4 \mathrm{~Hz}), 88.2,83.5,27.9,27.6$; IR $v_{\max }$ (neat) $/ \mathrm{cm}^{-1}: 3329,2986,1777,1729$, 1418, 1216, 1129, 1048, 1151, 604, 579; HRMS (ESI, m/z): calcd for $\mathrm{C}_{11} \mathrm{H}_{19} \mathrm{~F}_{3} \mathrm{~N}_{2} \mathrm{NaO}_{6} \mathrm{~S}^{+}[\mathrm{M}$ $+\mathrm{Na}]^{+}$387.0808, found 387.0810. 
Due to the presence of the two carbamate groups, 3a is probably conformationally constrained and shows broad absorption peaks on NMR spectra even at an elevated temperature. Because this phenomenon will cause difficulties in structure determination during substrates exploration, we decided to remove the protecting groups and characterize the hydrazinium hydrochloride salt instead.

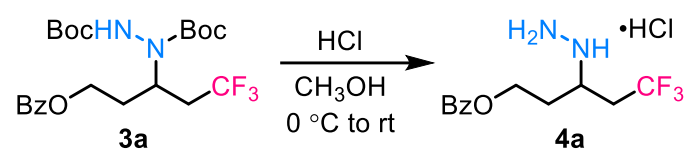

5,5,5-Trifluoro-3-hydrazinylpentyl benzoate hydrochloride (4a): To a $25 \mathrm{~mL}$ round bottom flask charged with a stir bar, 3a and $\mathrm{CH}_{3} \mathrm{OH}(2 \mathrm{~mL})$ was slowly added $\mathrm{HCl}\left(4 \mathrm{M}\right.$ in $\mathrm{CH}_{3} \mathrm{OH}$, $0.5 \mathrm{~mL}$ ) at $0{ }^{\circ} \mathrm{C}$. The reaction was then sealed with a PTFE septum and stirred at room temperature until the consumption of 3a. After the solvent was removed under reduced pressure, the residue was washed with anhydrous ether $(1 \mathrm{~mL} \times 3)$ and then dried in vacuo to afford the corresponding hydrazine hydrochloride $\mathbf{4 a}$ as colorless oil, (51 mg, 82\% yield). ${ }^{1} \mathrm{H}$ NMR (600 $\left.\mathrm{MHz}, \mathrm{D}_{2} \mathrm{O}\right) \delta 8.03-7.92(\mathrm{~m}, 2 \mathrm{H}), 7.62(\mathrm{~d}, J=7.5 \mathrm{~Hz}, 1 \mathrm{H}), 7.48(\mathrm{t}, J=7.8 \mathrm{~Hz}, 2 \mathrm{H}), 4.50-$ $4.39(\mathrm{~m}, 2 \mathrm{H}), 3.63(\mathrm{t}, J=6.1 \mathrm{~Hz}, 1 \mathrm{H}), 2.70-2.60(\mathrm{~m}, 2 \mathrm{H}), 2.22-2.11(\mathrm{~m}, 2 \mathrm{H}) .{ }^{19} \mathrm{~F} \mathrm{NMR}(376$ $\left.\mathrm{MHz}, \mathrm{D}_{2} \mathrm{O}\right) \delta-64.58(\mathrm{t}, J=11.0 \mathrm{~Hz}) ;{ }^{13} \mathrm{C}\left\{{ }^{1} \mathrm{H}\right\} \mathrm{NMR}\left(101 \mathrm{MHz}, \mathrm{CD}_{3} \mathrm{OD}\right) \delta 166.5,133.0,129.8$, $129.1,128.3,126.1(\mathrm{q}, J=273.6 \mathrm{~Hz}), 60.9,51.4,35.4(\mathrm{~d}, J=28.0 \mathrm{~Hz}), 30.9$; IR $v_{\max }($ neat $) / \mathrm{cm}^{-}$ ${ }^{1}:$ 2904, 1713, 1602, 1451, 1397, 1272, 1254, 1115, 1070, 710, 686; HRMS (ESI, m/z): calcd for $\mathrm{C}_{12} \mathrm{H}_{17} \mathrm{ClF}_{3} \mathrm{~N}_{2} \mathrm{O}_{2}{ }^{+}[\mathrm{M}+\mathrm{H}]^{+}$313.0931, found 313.0935.

\section{Trifluoromethyl-Hydrazination of Different Alkenes}
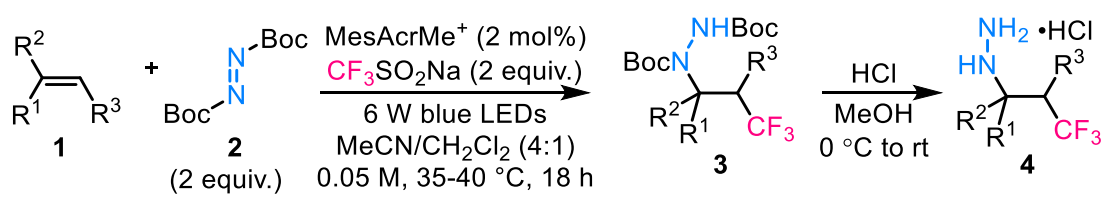

i. General procedure for preparation of trifluoromethylated hydrazinium salts $\mathbf{4}$ from corresponding alkenes:

To a sealable 2-dram vial equipped with a stir bar were added di-tert-butyl azodicarboxylate 2 (93 mg, $0.4 \mathrm{mmol})$ and $\mathrm{CF}_{3} \mathrm{SO}_{2} \mathrm{Na}(63 \mathrm{mg}, 0.4 \mathrm{mmol})$ and $\mathrm{MesAcrMe}^{+} \mathrm{ClO}_{4}{ }^{-}(1.6 \mathrm{mg}, 0.004$ mmol) in a glovebox. After the vial was taken out of the glovebox, anhydrous $\mathrm{CH}_{2} \mathrm{Cl}_{2}(0.8 \mathrm{~mL})$, 
$\mathrm{CH}_{3} \mathrm{CN}(3.2 \mathrm{~mL})$ and alkene $1(0.2 \mathrm{mmol})$ were added successively through syringes under $\mathrm{N}_{2}$. The reaction was then stirred under the irradiation of blue light (6 W LEDs, the photoreactor was maintained at between 35 to $40{ }^{\circ} \mathrm{C}$ by circulating water cooling). The reaction process was monitored with TLC. Upon the completion of reaction, the resulted mixture was quenched with saturated $\mathrm{NH}_{4} \mathrm{Cl}$ (aq., $\left.2 \mathrm{~mL}\right)$, extracted with EtOAc $(2 \mathrm{~mL} \times 3)$, dried over anhydrous $\mathrm{Na}_{2} \mathrm{SO}_{4}$ and concentrated in vacuo to afford the crude product, which was then purified through a silica gel flash column to afford a pure product 3. Compound 3 was then dissolved in $\mathrm{CH}_{3} \mathrm{OH}(2 \mathrm{~mL})$ in a round bottom flask, to which $\mathrm{HCl}\left(4 \mathrm{M}\right.$ in $\left.\mathrm{CH}_{3} \mathrm{OH}, 0.5 \mathrm{~mL}\right)$ was slowly added at $0{ }^{\circ} \mathrm{C}$. The reaction was then sealed with a PTFE septum and stirred at room temperature until the consumption of compound 3. After the solvent was removed under reduced pressure, the residue was washed with anhydrous ether $(1 \mathrm{~mL} \times 2)$ and then dried in vacuo to afford the corresponding hydrazine hydrochloride 4.

\section{ii. The characterization data of trifluoro-hydrazination products}

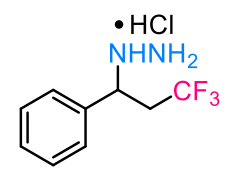

Styrene $\mathbf{1 b}$ is commercially available and it was distilled before use.

(3,3,3-Trifluoro-1-phenylpropyl)hydrazine hydrochloride (4b): compound $4 \mathrm{~b}$ was prepared from styrene by following the general procedure (foam, $35 \mathrm{mg}, 73 \%$ yield). ${ }^{1} \mathrm{H}$ NMR $\left(400 \mathrm{MHz}, \mathrm{D}_{2} \mathrm{O}\right) \delta 7.52-7.34(\mathrm{~m}, 5 \mathrm{H}), 4.40(\mathrm{t}, J=6.9 \mathrm{~Hz}, 1 \mathrm{H}), 2.88-2.67(\mathrm{~m}, 2 \mathrm{H}) ;{ }^{19} \mathrm{~F} \mathrm{NMR}$ $\left(376 \mathrm{MHz}, \mathrm{D}_{2} \mathrm{O}\right) \delta-63.28(\mathrm{t}, J=10.7 \mathrm{~Hz}) ;{ }^{13} \mathrm{C}\left\{{ }^{1} \mathrm{H}\right\} \mathrm{NMR}\left(101 \mathrm{MHz}, \mathrm{CD}_{3} \mathrm{OD}\right) \delta 136.7,129.1$, 129.0, 127.6, $125.6(\mathrm{q}, J=276.1 \mathrm{~Hz}), 58.2,38.2(\mathrm{q}, J=27.6 \mathrm{~Hz})$; IR $v_{\max }($ neat)/cm-1: 2980 , 1715, 1393, 1368, 1264, 1153, 735, 713; HRMS (ESI, m/z): calcd for $\mathrm{C}_{9} \mathrm{H}_{13} \mathrm{ClF}_{3} \mathrm{~N}_{2}{ }^{+}[\mathrm{M}+\mathrm{H}]^{+}$ 241.0514, found 241.0510.

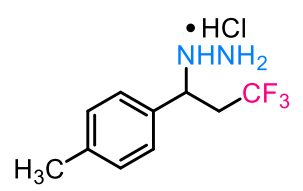

4-Methylstyrene 1c is commercially available and it was distilled before use. 
(3,3,3-Trifluoro-1-(p-tolyl)propyl)hydrazine hydrochloride (4c): compound 4c was prepared from 4-methylstyrene 1c by following the general procedure (foam, $33 \mathrm{mg}, 65 \%$ yield). ${ }^{1} \mathrm{H}$ NMR $\left(400 \mathrm{MHz}, \mathrm{D}_{2} \mathrm{O}\right) \delta 7.33-7.18(\mathrm{~m}, 4 \mathrm{H}), 4.33(\mathrm{t}, J=6.9 \mathrm{~Hz}, 1 \mathrm{H}), 2.84-2.61$ $(\mathrm{m}, 2 \mathrm{H}), 2.25(\mathrm{~s}, 3 \mathrm{H}) ;{ }^{19} \mathrm{~F}$ NMR $\left(376 \mathrm{MHz}, \mathrm{D}_{2} \mathrm{O}\right) \delta-63.36(\mathrm{t}, J=10.7 \mathrm{~Hz}) ;{ }^{13} \mathrm{C}\left\{{ }^{1} \mathrm{H}\right\} \mathrm{NMR}(101$ $\left.\mathrm{MHz}, \mathrm{CD}_{3} \mathrm{OD}\right) \delta 139.2,133.5,129.6,127.6,125.6(\mathrm{q}, J=276.1 \mathrm{~Hz}), 58.0,38.1(\mathrm{q}, J=28.2$ Hz), 19.9; IR $v_{\max }$ (neat) $/ \mathrm{cm}^{-1}: 2930,1698,1612,1515,1373,1262,1137,737$, 702; HRMS (ESI, m/z): calcd for $\mathrm{C}_{10} \mathrm{H}_{15} \mathrm{ClF}_{3} \mathrm{~N}_{2}^{+}[\mathrm{M}+\mathrm{H}]^{+}$255.0871, found 255.0865.

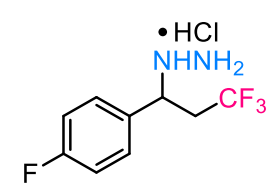

4-Fluorostyrene $\mathbf{1 d}$ is commercially available and it was distilled before use.

(3,3,3-Trifluoro-1-(4-fluorophenyl)propyl)hydrazine hydrochloride (4d): compound 4d was prepared from 1-fluoro-4-vinylbenzene 1d by following the general procedure (foam, 42 $\mathrm{mg}, 82 \%$ yield). ${ }^{1} \mathrm{H}$ NMR (400 MHz, $\left.\mathrm{D}_{2} \mathrm{O}\right) \delta 7.47-7.32(\mathrm{~m}, 2 \mathrm{H}), 7.10(\mathrm{t}, J=8.6 \mathrm{~Hz}, 2 \mathrm{H}), 4.36$ $(\mathrm{t}, J=6.9 \mathrm{~Hz}, 1 \mathrm{H}), 2.82-2.56(\mathrm{~m}, 2 \mathrm{H}) ;{ }^{19} \mathrm{~F} \mathrm{NMR}\left(376 \mathrm{MHz}, \mathrm{D}_{2} \mathrm{O}\right) \delta-63.34(\mathrm{t}, J=10.7 \mathrm{~Hz}),-$ $112.61(\mathrm{t}, J=5.2 \mathrm{~Hz}) ;{ }^{13} \mathrm{C}\left\{{ }^{1} \mathrm{H}\right\} \operatorname{NMR}\left(151 \mathrm{MHz}, \mathrm{D}_{2} \mathrm{O}\right) \delta 163.1(\mathrm{~d}, J=245.9 \mathrm{~Hz}), 131.7(\mathrm{~d}, J$ $=3.1 \mathrm{~Hz}), 130.0(\mathrm{~d}, J=8.7 \mathrm{~Hz}), 125.4(\mathrm{q}, J=276.3 \mathrm{~Hz}), 116.3(\mathrm{~d}, J=21.8 \mathrm{~Hz}), 57.2,37.6(\mathrm{q}$, $J=28.3 \mathrm{~Hz}$ ); IR $v_{\max }($ neat)/cm-1: 2950, 1697, 1598, 1508, 1370, 1225, 1094, 998, 836, 593; HRMS (ESI, m/z): calcd for $\mathrm{C}_{9} \mathrm{H}_{12} \mathrm{ClF}_{4} \mathrm{~N}_{2}{ }^{+}[\mathrm{M}+\mathrm{H}]^{+}$259.0620, found 259.0624.

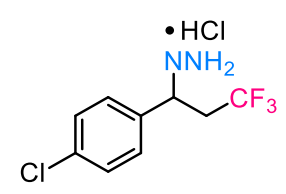

4-Chlorostyrene $\mathbf{1 e}$ is commercially available and it was distilled before use.

(1-(4-Chlorophenyl)-3,3,3-trifluoropropyl)hydrazine hydrochloride (4e): compound 4e was prepared from 4-chlorostyrene 1e by following the general procedure (white solid, $38 \mathrm{mg}$, $70 \%$ yield, b.p. $\left.165.1-165.2{ }^{\circ} \mathrm{C}\right) .{ }^{1} \mathrm{H}$ NMR $\left(400 \mathrm{MHz}, \mathrm{D}_{2} \mathrm{O}\right) \delta 7.57-7.27(\mathrm{~m}, 4 \mathrm{H}), 4.35(\mathrm{t}, J$ $=6.9 \mathrm{~Hz}, 1 \mathrm{H}), 2.84-2.56(\mathrm{~m}, 2 \mathrm{H}) ;{ }^{19} \mathrm{~F} \mathrm{NMR}\left(376 \mathrm{MHz}, \mathrm{D}_{2} \mathrm{O}\right) \delta-63.34(\mathrm{t}, J=10.8 \mathrm{~Hz}) ;{ }^{13} \mathrm{C}\left\{{ }^{1} \mathrm{H}\right\}$ NMR (101 MHz, $\left.\mathrm{D}_{2} \mathrm{O}\right) \delta 134.9,134.6,129.5,129.4,125.4$ (q, $\left.J=276.6 \mathrm{~Hz}\right), 57.2$ (q, $J=3.0$ Hz), 37.6 (q, $J=28.4$ Hz); IR $v_{\max }($ neat)/cm-1: 2885, 1681, 1516, 1393, 1262, 1138, 1118, 1097 , 824, 735, 701; HRMS (ESI, m/z): calcd for $\mathrm{C}_{9} \mathrm{H}_{11} \mathrm{Cl}_{2} \mathrm{~F}_{3} \mathrm{~N}_{2}{ }^{+}[\mathrm{M}+\mathrm{H}]^{+} 274.0246$, found 274.0240. 


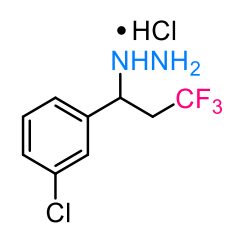

3-Chlorostyrene $\mathbf{1 f}$ is commercially available and it was distilled before use.

(1-(3-Chlorophenyl)-3,3,3-trifluoropropyl)hydrazine hydrochloride (4f): compound 4f was prepared from 3-chlorostyrene 1f by following the general procedure (foam, $33 \mathrm{mg}, 60 \%$ yield). ${ }^{1} \mathrm{H}$ NMR (400 MHz, $\left.\mathrm{D}_{2} \mathrm{O}\right) \delta 7.42(\mathrm{~s}, 1 \mathrm{H}), 7.38-7.21(\mathrm{~m}, 3 \mathrm{H}), 4.34(\mathrm{t}, J=6.9 \mathrm{~Hz}, 1 \mathrm{H})$, $2.83-2.55(\mathrm{~m}, 2 \mathrm{H}) ;{ }^{19} \mathrm{~F}$ NMR $\left(376 \mathrm{MHz}, \mathrm{D}_{2} \mathrm{O}\right) \delta-63.22(\mathrm{t}, J=10.2 \mathrm{~Hz}) ;{ }^{13} \mathrm{C}\left\{{ }^{1} \mathrm{H}\right\} \mathrm{NMR}(101$ $\left.\mathrm{MHz}, \mathrm{D}_{2} \mathrm{O}\right) \delta 138.2,134.5,131.0,129.7,127.9,128.1,125.4(\mathrm{q}, J=277.1 \mathrm{~Hz}), 57.4,37.6$ (q, $J=28.6 \mathrm{~Hz}$ ); IR $v_{\max }\left(\right.$ neat) $/ \mathrm{cm}^{-1}: 2891,1685,1510,1393,1260,1141,1118,1091,820,731$, 707; HRMS (ESI, m/z): calcd for $\mathrm{C}_{9} \mathrm{H}_{11} \mathrm{Cl}_{2} \mathrm{~F}_{3} \mathrm{~N}_{2}{ }^{+}[\mathrm{M}+\mathrm{H}]^{+} 274.0246$, found 274.0241.

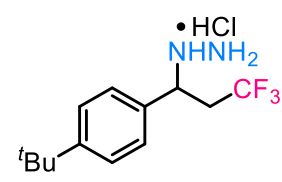

4-tert-Butylstyrene $\mathbf{1 g}$ is commercially available and it was distilled before use.

(1-(4-(tert-Butyl)phenyl)-3,3,3-trifluoropropyl)hydrazine hydrochloride (4g): compound $\mathbf{4 g}$ was prepared from 4-tert-butylstyrene $1 \mathbf{g}$ by following the general procedure (foam, $45 \mathrm{mg}$, $76 \%$ yield). ${ }^{1} \mathrm{H}$ NMR (400 MHz, $\left.\mathrm{D}_{2} \mathrm{O}\right) \delta 7.47(\mathrm{~d}, J=8.2 \mathrm{~Hz}, 2 \mathrm{H}), 7.32(\mathrm{~d}, J=8.3 \mathrm{~Hz}, 2 \mathrm{H})$, $4.34(\mathrm{t}, J=6.9 \mathrm{~Hz}, 1 \mathrm{H}), 2.90-2.59(\mathrm{~m}, 2 \mathrm{H}), 1.19(\mathrm{~s}, 9 \mathrm{H}) ;{ }^{19} \mathrm{~F} \mathrm{NMR}\left(376 \mathrm{MHz}, \mathrm{D}_{2} \mathrm{O}\right) \delta-63.37$ $(\mathrm{t}, J=10.7 \mathrm{~Hz}) ;{ }^{13} \mathrm{C}\left\{{ }^{1} \mathrm{H}\right\} \mathrm{NMR}\left(101 \mathrm{MHz}, \mathrm{CD}_{3} \mathrm{OD}\right) \delta 152.3,133.7,127.3,126.0,125.8(\mathrm{q}, J$ $=277.1 \mathrm{~Hz}), 57.9,38.2(\mathrm{q}, J=28.2 \mathrm{~Hz}), 34.1,30.3$; IR $v_{\max }($ neat $) / \mathrm{cm}^{-1}: 2965,1693,1605,1515$, 1416, 1367, 1266, 1134, 1104, 997, 739, 641; HRMS (ESI, m/z): calcd for $\mathrm{C}_{13} \mathrm{H}_{21} \mathrm{ClF}_{3} \mathrm{~N}_{2}{ }^{+}[\mathrm{M}$ $+\mathrm{H}]^{+}$297.1340, found 297.1346.

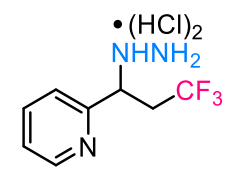

2-Vinylpyridine $\mathbf{1 h}$ is commercially available and it was distilled before use.

2-(3,3,3-trifluoro-1-hydrazinylpropyl)pyridine hydrochloride (4h): compound $4 \mathrm{~h}$ was prepared from 2-vinylpyridine $\mathbf{1 h}$ by following the general procedure (foam, $41 \mathrm{mg}, 73 \%$ yield). 
${ }^{1} \mathrm{H}$ NMR $\left(400 \mathrm{MHz}, \mathrm{D}_{2} \mathrm{O}\right) \delta 8.69(\mathrm{~d}, J=5.8 \mathrm{~Hz}, 1 \mathrm{H}), 8.56-8.44(\mathrm{~m}, 1 \mathrm{H}), 8.10(\mathrm{~d}, J=8.0 \mathrm{~Hz}$, $1 \mathrm{H}), 8.00-7.90(\mathrm{~m}, 1 \mathrm{H}), 4.86(\mathrm{t}, J=6.6 \mathrm{~Hz}, 1 \mathrm{H}), 3.02-2.62(\mathrm{~m}, 2 \mathrm{H}),{ }^{19} \mathrm{~F}$ NMR $(376 \mathrm{MHz}$, $\left.\mathrm{D}_{2} \mathrm{O}\right) \delta-63.14(\mathrm{~d}, J=8.8 \mathrm{~Hz}) ;{ }^{13} \mathrm{C}\left\{{ }^{1} \mathrm{H}\right\} \mathrm{NMR}\left(101 \mathrm{MHz}, \mathrm{D}_{2} \mathrm{O}\right) \delta 150.9,148.1,142.7,127.9$, 127.3, 124.7 (q, $J=276.7 \mathrm{~Hz}), 55.1,37.0$ (q, $J=29.5 \mathrm{~Hz})$; IR $v_{\max }($ neat)/cm-1: 2924, 1714, 1704, 1633, 1455, 1393, 1368, 1256, 1153, 740, 681; HRMS (ESI, m/z): calcd for $\mathrm{C}_{8} \mathrm{H}_{12} \mathrm{ClF}_{3} \mathrm{~N}_{3}{ }^{+}[\mathrm{M}+\mathrm{H}]^{+} 242.0667$, found 242.0670.

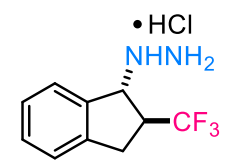

Indene $\mathbf{1 i}$ is commercially available and it was distilled before use.

(2-(Trifluoromethyl)-2,3-dihydro-1H-inden-1-yl)hydrazine hydrochloride (4i): compound $4 \mathbf{i}$ was prepared from indene 1i by following the general procedure (white solid, $35 \mathrm{mg}, 69 \%$ yield, m.p. $\left.140.1-140.2{ }^{\circ} \mathrm{C}\right) .{ }^{1} \mathrm{H}$ NMR $\left(400 \mathrm{MHz}, \mathrm{D}_{2} \mathrm{O}\right) \delta 7.45(\mathrm{~d}, J=7.6 \mathrm{~Hz}, 1 \mathrm{H}), 7.42-$ $7.27(\mathrm{~m}, 3 \mathrm{H}), 5.03-4.96(\mathrm{~m}, 1 \mathrm{H}), 3.49-3.29(\mathrm{~m}, 2 \mathrm{H}), 3.16(\mathrm{~d}, J=13.8 \mathrm{~Hz}, 1 \mathrm{H}) ;{ }^{19} \mathrm{~F}$ NMR $\left(376 \mathrm{MHz}, \mathrm{D}_{2} \mathrm{O}\right) \delta-71.55(\mathrm{~d}, J=8.8 \mathrm{~Hz}) ;{ }^{13} \mathrm{C}\left\{{ }^{1} \mathrm{H}\right\} \mathrm{NMR}\left(101 \mathrm{MHz}, \mathrm{CD}_{3} \mathrm{OD}\right) \delta 142.4,135.2$, $130.5,127.8,127.2$ (q, $J=277.4 \mathrm{~Hz}), 125.36,125.34,64.6,45.2$ (q, $J=27.1 \mathrm{~Hz}), 30.9$; IR $v_{\max }$ (neat)/cm ${ }^{-1}: 2932,1714,1394,1373,1263,1161,1111,732,702 ;$ HRMS (ESI, m/z): calcd for $\mathrm{C}_{10} \mathrm{H}_{13} \mathrm{ClF}_{3} \mathrm{~N}_{2}{ }^{+}[\mathrm{M}+\mathrm{H}]^{+}$253.0719, found 253.0723.

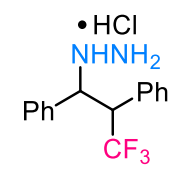

trans-Stilbene $\mathbf{1 j}$ is commercially available and it was recrystallized from ethanol before use.

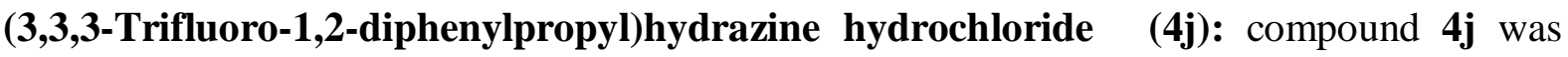
prepared from trans-stilbene $\mathbf{1 j}$ by following the general procedure (foam, $46 \mathrm{mg}, 73 \%$ yield). ${ }^{1} \mathrm{H}$ NMR (400 MHz, D $\left.2 \mathrm{O}\right) \delta 7.48$ - 7.36 (m, 2H), 7.16 (s, 4H), 7.11 (s, 4H), 4.65 (d, J = 10.6 $\mathrm{Hz}, 1 \mathrm{H}), 4.12-3.90(\mathrm{~m}, 1 \mathrm{H}) ;{ }^{19} \mathrm{~F}$ NMR $\left(376 \mathrm{MHz}, \mathrm{D}_{2} \mathrm{O}\right) \delta-63.87$ (d, $\left.J=8.9 \mathrm{~Hz}\right)$ (major), $64.11(\mathrm{~d}, J=8.8 \mathrm{~Hz})$ (minor); ${ }^{13} \mathrm{C}\left\{{ }^{1} \mathrm{H}\right\} \mathrm{NMR}\left(101 \mathrm{MHz}, \mathrm{CD}_{3} \mathrm{OD}\right) \delta 135.9$ (minor), 135.3 (major), 132.4 (major), 131.9 (minor), 129.3 (major), 129.1 (minor), 128.9 (major+minor), 128.72 (major), 128.66 (minor), 128.6 (minor), 128.53 (major), 128.46 (major), 128.3 (minor), 
128.0 (major), 127.8 (minor), 126.3 (q, $J=278.4 \mathrm{~Hz}$ ) (major), 125.6 (q, $J=278.4 \mathrm{~Hz}$ ) (minor), 63.6 (major), 62.6 (minor), $54.8\left(\mathrm{~d}, J=25.7 \mathrm{~Hz}\right.$ ) (minor), 53.8 (q, $J=26.2 \mathrm{~Hz}$ ) (major); IR $v_{\max }$ (neat) $/ \mathrm{cm}^{-1}: 3033,1691,1597,1496,1455,1362,1253,1149,1113,892,837,756,699,650$, 615, 594; HRMS (ESI, m/z): calcd for $\mathrm{C}_{15} \mathrm{H}_{17} \mathrm{ClF}_{3} \mathrm{~N}_{2}{ }^{+}[\mathrm{M}+\mathrm{H}]^{+}$317.1032, found 317.1035.

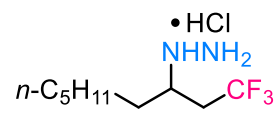

1-Octene $1 \mathbf{k}$ is commercially available and it was distilled before use.

(1,1,1-Trifluorononan-3-yl)hydrazine hydrochloride (4k): compound 4k was prepared from 1-octene $1 \mathbf{k}$ by following the general procedure (colorless oil, $40 \mathrm{mg}, 80 \%$ yield). ${ }^{1} \mathrm{H}$ NMR (600 MHz, $\left.\mathrm{D}_{2} \mathrm{O}\right) \delta 3.46-3.34(\mathrm{~m}, 1 \mathrm{H}), 2.63-2.45(\mathrm{~m}, 2 \mathrm{H}), 1.69-1.52$ (m, 2H), $1.30-1.19$ $(\mathrm{m}, 4 \mathrm{H}), 1.18-1.12(\mathrm{~m}, 4 \mathrm{H}), 0.76-0.69(\mathrm{~m}, 3 \mathrm{H}) ;{ }^{19} \mathrm{~F} \mathrm{NMR}\left(565 \mathrm{MHz}, \mathrm{D}_{2} \mathrm{O}\right) \delta-63.48(\mathrm{t}, J=$ 11.2 Hz); ${ }^{13} \mathrm{C}\left\{{ }^{1} \mathrm{H}\right\}$ NMR (101 MHz, $\left.\mathrm{D}_{2} \mathrm{O}\right) \delta 125.9(\mathrm{q}, J=276.2 \mathrm{~Hz}), 54.9(\mathrm{q}, J=2.6 \mathrm{~Hz}), 33.7$ (q, $J=28.6 \mathrm{~Hz}), 30.7,30.0,28.0,23.9,21.8,13.3$; IR $v_{\max }$ (neat) $/ \mathrm{cm}^{-1}: 2929,1704,1456,1392$, 1367, 1262, 1152, 1047, 1019, 940, 855, 736, 703; HRMS (ESI, m/z): calcd for $\mathrm{C}_{9} \mathrm{H}_{21} \mathrm{ClF}_{3} \mathrm{~N}_{2}{ }^{+}$ $[\mathrm{M}+\mathrm{H}]^{+}$249.1345, found 249.1350.

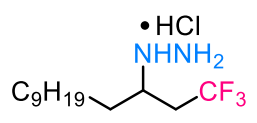

1-Dodecene $\mathbf{1 l}$ is commercially available and it was distilled before use.

(1,1,1-trifluorotridecan-3-yl)hydrazine hydrochloride (4l): compound 41 was prepared from 1-dodecene 11 by following the general procedure (colorless oil, $45 \mathrm{mg}, 83 \%$ yield). ${ }^{1} \mathrm{H}$ NMR (400 MHz, CD 3 OD) $\delta 3.32-3.05(\mathrm{~m}, 1 \mathrm{H}), 2.77-2.44(\mathrm{~m}, 1 \mathrm{H}), 2.45-2.27(\mathrm{~m}, 1 \mathrm{H}), 1.78-$ $1.45(\mathrm{~m}, 2 \mathrm{H}), 1.40-1.08(\mathrm{~m}, 16 \mathrm{H}), 0.80(\mathrm{t}, J=6.6 \mathrm{~Hz}, 3 \mathrm{H}) ;{ }^{19} \mathrm{~F}$ NMR $\left(376 \mathrm{MHz}, \mathrm{CD}_{3} \mathrm{OD}\right) \delta-$ $64.72(\mathrm{t}, J=11.1 \mathrm{~Hz}) ;{ }^{13} \mathrm{C}\left\{{ }^{1} \mathrm{H}\right\} \mathrm{NMR}\left(101 \mathrm{MHz}, \mathrm{CD}_{3} \mathrm{OD}\right) \delta 126.2(\mathrm{q}, J=276.2 \mathrm{~Hz}), 54.3,34.7$ (q, $J=28.2 \mathrm{~Hz}), 31.7,31.1,29.29,29.27,29.11,29.09,29.0,24.5,22.3,13.1$; IR $v_{\max }\left(\right.$ neat) $/ \mathrm{cm}^{-}$ 1: 2924, 2854, 1731, 1601, 1509, 1466, 1254, 1145, 740; HRMS (ESI, m/z): calcd for $\mathrm{C}_{13} \mathrm{H}_{29} \mathrm{ClF}_{3} \mathrm{~N}_{2}{ }^{+}[\mathrm{M}+\mathrm{H}]^{+}$305.1971, found 305.1976.

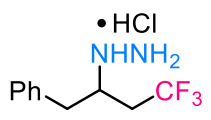

Allylbenzene $\mathbf{1 m}$ is commercially available and it was distilled before use. 
(4,4,4-Trifluoro-1-phenylbutan-2-yl)hydrazine hydrochloride $(\mathbf{4 m})$ : compound $\mathbf{4 m}$ was prepared from allylbenzene $1 \mathrm{~m}$ by following the general procedure (foam, $31 \mathrm{mg}, 72 \%$ yield). ${ }^{1} \mathrm{H}$ NMR $\left(400 \mathrm{MHz}, \mathrm{D}_{2} \mathrm{O}\right) \delta 7.35-7.23(\mathrm{~m}, 2 \mathrm{H}), 7.23-7.08(\mathrm{~m}, 3 \mathrm{H}), 3.80-3.46(\mathrm{~m}, 1 \mathrm{H})$, $2.95(\mathrm{dd}, J=14.1,6.7 \mathrm{~Hz}, 1 \mathrm{H}), 2.81(\mathrm{dd}, J=14.1,7.9 \mathrm{~Hz}, 1 \mathrm{H}), 2.57-2.25(\mathrm{~m}, 2 \mathrm{H}) ;{ }^{19} \mathrm{~F} \mathrm{NMR}$ $\left(376 \mathrm{MHz}, \mathrm{D}_{2} \mathrm{O}\right) \delta-63.22(\mathrm{t}, J=11.1 \mathrm{~Hz}) ;{ }^{13} \mathrm{C}\left\{{ }^{1} \mathrm{H}\right\} \mathrm{NMR}\left(101 \mathrm{MHz}, \mathrm{D}_{2} \mathrm{O}\right) \delta 135.5,129.5$, $129.1,127.5,125.9$ (q, $J=272.6 \mathrm{~Hz}), 55.5(\mathrm{q}, J=2.5 \mathrm{~Hz}), 36.9,33.9(\mathrm{q}, J=28.7 \mathrm{~Hz})$; IR $v_{\max }$ (neat) $/ \mathrm{cm}^{-1}: 2924,1585,1496,1455,1393,1253,1135,919,793,738,699,663$; HRMS (ESI, $\mathrm{m} / \mathrm{z}$ ): calcd for $\mathrm{C}_{10} \mathrm{H}_{15} \mathrm{ClF}_{3} \mathrm{~N}_{2}{ }^{+}[\mathrm{M}+\mathrm{H}]^{+}$255.0876, found 255.0872 .

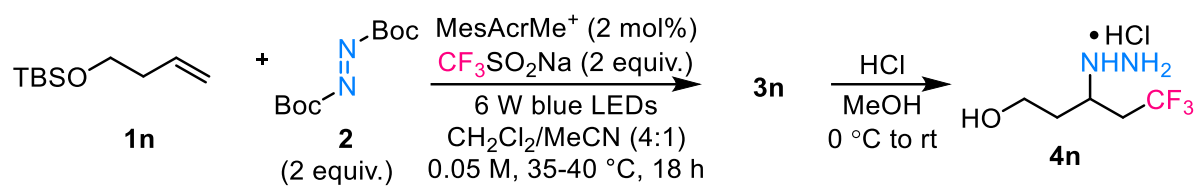

(But-3-en-1-yloxy)(tert-butyl)dimethylsilane 1n is a known compound and it was prepared according to a literature procedure. ${ }^{[2]}$

5,5,5-Trifluoro-3-hydrazinylpentan-1-ol hydrochloride (4n): compound 4n was prepared from (but-3-en-1-yloxy)(tert-butyl)dimethylsilane 1n by following the general procedure (colorless oil, $38 \mathrm{mg}, 92 \%$ yield). ${ }^{1} \mathrm{H}$ NMR (400 MHz, $\left.\mathrm{CD}_{3} \mathrm{OD}\right) \delta 3.87-3.76(\mathrm{~m}, 1 \mathrm{H}), 3.76-$ $3.64(\mathrm{~m}, 1 \mathrm{H}), 3.57-3.44(\mathrm{~m}, 1 \mathrm{H}), 2.77-2.42(\mathrm{~m}, 2 \mathrm{H}), 2.01-1.79(\mathrm{~m}, 2 \mathrm{H}) ;{ }^{19} \mathrm{~F}$ NMR $(376$ $\left.\mathrm{MHz}, \mathrm{CD}_{3} \mathrm{OD}\right) \delta-64.61(\mathrm{t}, J=10.9 \mathrm{~Hz}) ;{ }^{13} \mathrm{C}\left\{{ }^{1} \mathrm{H}\right\} \mathrm{NMR}\left(151 \mathrm{MHz}, \mathrm{CD}_{3} \mathrm{OD}\right) \delta 126.1(\mathrm{q}, J=$ $276.2 \mathrm{~Hz}$ ), 58.1, 53.5, 35.5 (q, $J=30.4 \mathrm{~Hz}$ ), 33.7; IR $v_{\max }$ (neat) $/ \mathrm{cm}^{-1}: 2978,2929,1703,1454$, 1365, 1256, 1129, 1083, 738; HRMS (ESI, m/z): calcd for $\mathrm{C}_{5} \mathrm{H}_{13} \mathrm{ClF}_{3} \mathrm{~N}_{2} \mathrm{O}^{+}[\mathrm{M}+\mathrm{H}]^{+} 209.0669$, found 209.0665 .

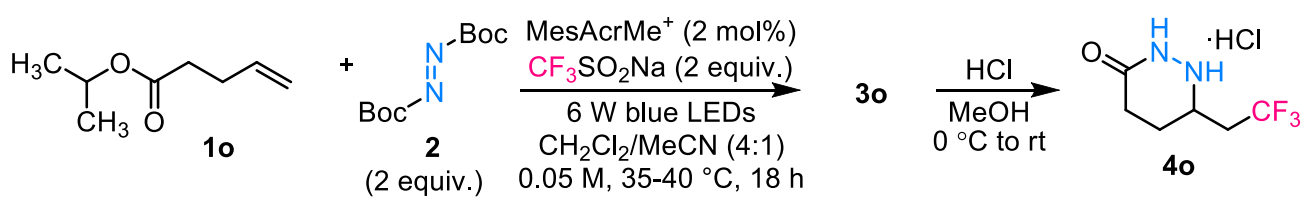

Isopropyl pent-4-enoate 10 is a known compound and it was prepared according to a literature procedure. $^{[3]}$

6-(2,2,2-Trifluoroethyl)tetrahydropyridazin-3(2H)-one hydrochloride (4o): compound 40 was prepared from isopropyl pent-4-enoate 10 by following the general procedure (foam, 36 mg, 82\%). ${ }^{1} \mathrm{H}$ NMR (400 MHz, $\left.\mathrm{D}_{2} \mathrm{O}\right) \delta 4.11-3.98$ (m, 1H), 2.86 - 2.63 (m, 1H), 2.56 - 2.26 $(\mathrm{m}, 4 \mathrm{H}), 2.03-1.87(\mathrm{~m}, 1 \mathrm{H}) ;{ }^{19} \mathrm{~F}$ NMR $\left(376 \mathrm{MHz}, \mathrm{CD}_{3} \mathrm{OD}\right) \delta-63.34(\mathrm{t}, J=11.3 \mathrm{~Hz}) ;{ }^{13} \mathrm{C}\left\{{ }^{1} \mathrm{H}\right\}$ 
NMR (101 MHz, CD 3 OD) $\delta$ 173.7, 125.8 (q, $J=276.7 \mathrm{~Hz}), 53.4,36.2$ (q, $J=27.6 \mathrm{~Hz}), 26.3$, 23.3; IR $v_{\max }$ (neat)/cm ${ }^{-1}: 2925,1729,1682,1516,1395,1354,1253,1145,1106,1026,735$, 646; HRMS (ESI, m/z): calcd for $\mathrm{C}_{6} \mathrm{H}_{11} \mathrm{ClF}_{3} \mathrm{~N}_{2} \mathrm{O}^{+}[\mathrm{M}+\mathrm{H}]^{+}$219.0512, found 219.0515.

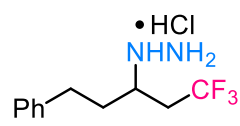

4-Phenyl-1-butene 1p is commercially available and it was distilled before use.

(1,1,1-Trifluoro-5-phenylpentan-3-yl)hydrazine hydrochloride (4p): compound $4 p$ was prepared from 4-phenyl-1-butene 1p by following the general procedure (colorless oil, $42 \mathrm{mg}$, $78 \%$ yield). ${ }^{1} \mathrm{H}$ NMR (400 MHz, $\left.\mathrm{CD}_{3} \mathrm{OD}\right) \delta 6.96-6.85(\mathrm{~m}, 4 \mathrm{H}), 6.86-6.77(\mathrm{~m}, 1 \mathrm{H}), 3.11-$ $2.71(\mathrm{~m}, 1 \mathrm{H}), 2.39(\mathrm{t}, J=8.1 \mathrm{~Hz}, 2 \mathrm{H}), 2.34-2.11(\mathrm{~m}, 2 \mathrm{H}), 1.70-1.47(\mathrm{~m}, 2 \mathrm{H}) ;{ }^{19} \mathrm{~F}$ NMR $(376$ $\left.\mathrm{MHz}, \mathrm{CD}_{3} \mathrm{OD}\right) \delta-64.53(\mathrm{t}, J=11.3 \mathrm{~Hz}) ;{ }^{13} \mathrm{C}\left\{{ }^{1} \mathrm{H}\right\} \mathrm{NMR}\left(101 \mathrm{MHz}, \mathrm{CD}_{3} \mathrm{OD}\right) \delta 141.8,129.3$, $129.2,127.4(\mathrm{q}, J=276.8 \mathrm{~Hz}), 127.0,54.9,36.0(\mathrm{q}, J=28.0 \mathrm{~Hz}), 34.4,31.9$; IR $v_{\max }($ neat $) / \mathrm{cm}^{-}$ 1: 2925, 1729, 1682, 1516, 1395, 1354, 1253, 1145, 1026, 735, 646; HRMS (ESI, m/z): calcd for $\mathrm{C}_{11} \mathrm{H}_{17} \mathrm{ClF}_{3} \mathrm{~N}_{2}{ }^{+}[\mathrm{M}+\mathrm{H}]^{+}$269.1032, found 269.1030.

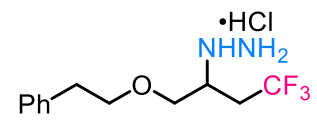

(2-(Allyloxy)ethyl)benzene 1q is a known compound and it was prepared according to a literature procedure. ${ }^{[4]}$

(4,4,4-Trifluoro-1-phenethoxybutan-2-yl)hydrazine hydrochloride (4q): compound $\mathbf{4 q}$ was prepared (2-(allyloxy)ethyl)benzene 1q by following the general procedure (foam, $42 \mathrm{mg}$, $71 \%$ yield). ${ }^{1} \mathrm{H}$ NMR (400 MHz, $\left.\mathrm{CD}_{3} \mathrm{OD}\right) \delta 7.42-7.34(\mathrm{~m}, 2 \mathrm{H}), 7.34-7.23(\mathrm{~m}, 3 \mathrm{H}), 3.88-$ $3.71(\mathrm{~m}, 3 \mathrm{H}), 3.69-3.61(\mathrm{~m}, 1 \mathrm{H}), 3.61-3.54(\mathrm{~m}, 1 \mathrm{H}), 2.94(\mathrm{t}, J=6.6 \mathrm{~Hz}, 2 \mathrm{H}), 2.60-2.37$ $(\mathrm{m}, 2 \mathrm{H}) ;{ }^{19} \mathrm{~F}$ NMR $\left(376 \mathrm{MHz}, \mathrm{CD}_{3} \mathrm{OD}\right) \delta-64.10(\mathrm{t}, J=11.1 \mathrm{~Hz}) ;{ }^{13} \mathrm{C}\left\{{ }^{1} \mathrm{H}\right\} \mathrm{NMR}(101 \mathrm{MHz}$, $\left.\mathrm{D}_{2} \mathrm{O}\right) \delta 140.4,130.2,129.9,127.8,127.3(\mathrm{q}, J=275.9 \mathrm{~Hz}), 73.4,72.2,54.5(\mathrm{q}, J=2.6 \mathrm{~Hz})$, 36.6, $34.0(\mathrm{q}, J=29.1 \mathrm{~Hz})$; IR $v_{\max }\left(\right.$ neat) $/ \mathrm{cm}^{-1}: 2960,1455,1208,1117,1085,967,823$; HRMS (ESI, m/z): calcd for $\mathrm{C}_{12} \mathrm{H}_{19} \mathrm{ClF}_{3} \mathrm{~N}_{2} \mathrm{O}^{+}[\mathrm{M}+\mathrm{H}]^{+} 299.1138$, found 299.1135.

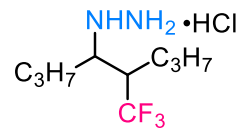

trans-4-Octene $\mathbf{1 r}$ is commercially available and it was distilled before use. 
(5-(Trifluoromethyl)octan-4-yl)hydrazine hydrochloride (4r): compound 4r was prepared from trans-4-octene $1 \mathbf{r}$ by following the general procedure (colorless oil, $32 \mathrm{mg}, 65 \%$ yield, $d r$ = 1.2). ${ }^{1} \mathrm{H}$ NMR (400 MHz, CD $\left.{ }_{3} \mathrm{OD}\right) \delta 3.33-3.15(\mathrm{~m}, 1 \mathrm{H}), 2.74-2.51(\mathrm{~m}, 1 \mathrm{H}), 1.71-1.40$ $(\mathrm{m}, 4 \mathrm{H}), 1.40-1.22(\mathrm{~m}, 4 \mathrm{H}), 0.90-0.73(\mathrm{~m}, 6 \mathrm{H}),{ }^{19} \mathrm{~F} \mathrm{NMR}\left(376 \mathrm{MHz}, \mathrm{CD}_{3} \mathrm{OD}\right) \delta-65.60(\mathrm{~d}$, $J=9.8 \mathrm{~Hz}$, isomer 1), $-65.93(\mathrm{~d}, J=9.9 \mathrm{~Hz}$, isomer 2$),{ }^{13} \mathrm{C}\left\{{ }^{1} \mathrm{H}\right\} \mathrm{NMR}\left(101 \mathrm{MHz}, \mathrm{D}_{2} \mathrm{O}\right) \delta 127.6$ $(\mathrm{q}, J=281.4 \mathrm{~Hz}), 127.5(\mathrm{q}, J=281.4 \mathrm{~Hz}), 59.6,59.1,42.5(\mathrm{q}, J=24.1 \mathrm{~Hz}), 42.1(\mathrm{q}, J=24.6$ Hz), 29.7, 26.04, 25.95, 20.3, 20.2, 19.3, 18.9, 13.2), 13.1, 12.98, 12.95; IR $v_{\max }$ (neat) $/ \mathrm{cm}^{-1}$ : 2964, 1603, 1533, 1470, 1263, 1244, 1154, 737, 701; HRMS (ESI, m/z): calcd for $\mathrm{C}_{9} \mathrm{H}_{21} \mathrm{ClF}_{3} \mathrm{~N}_{2}{ }^{+}[\mathrm{M}+\mathrm{H}]^{+}$249.1345, found 249.1350.

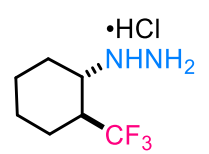

Cyclohexene 1s is commercially available and it was distilled before use.

(2-(Trifluoromethyl)cyclohexyl)hydrazine hydrochloride (4s): compound $4 \mathbf{s}$ was prepared from cyclohexene 1s by following the general procedure (white solid, $31 \mathrm{mg}, 71 \%$ yield, m.p. 166.2 -166.3 $\left.{ }^{\circ} \mathrm{C}\right) .{ }^{1} \mathrm{H}$ NMR $\left(600 \mathrm{MHz}, \mathrm{D}_{2} \mathrm{O}\right) \delta 3.35-3.24(\mathrm{~m}, 1 \mathrm{H}), 2.56-2.35(\mathrm{~m}, 1 \mathrm{H}), 2.12$ $-2.04(\mathrm{~m}, 1 \mathrm{H}), 2.06-1.98(\mathrm{~m}, 1 \mathrm{H}), 1.84-1.76(\mathrm{~m}, 1 \mathrm{H}), 1.72(\mathrm{~d}, J=12.7 \mathrm{~Hz}, 1 \mathrm{H}), 1.44-$ $1.30(\mathrm{~m}, 2 \mathrm{H}), 1.30-1.15(\mathrm{~m}, 2 \mathrm{H}) ;{ }^{19} \mathrm{~F}$ NMR $\left(376 \mathrm{MHz}, \mathrm{D}_{2} \mathrm{O}\right) \delta-68.32(\mathrm{~d}, J=8.6 \mathrm{~Hz}) ;{ }^{13} \mathrm{C}\left\{{ }^{1} \mathrm{H}\right\}$ $\operatorname{NMR}\left(101 \mathrm{MHz}, \mathrm{CD}_{3} \mathrm{OD}\right) \delta 127.0(\mathrm{q}, J=279.7 \mathrm{~Hz}), 56.2,43.3(\mathrm{q}, J=25.2 \mathrm{~Hz}) ; 28.2,24.0$ (q, $J=3.2 \mathrm{~Hz}$ ) 23.3, 23.0; IR $v_{\max }\left(\right.$ neat) $/ \mathrm{cm}^{-1}: 2939,1714,1594,1454,1334,1260,1140,1090$, 735, 690; HRMS (ESI, m/z): calcd for $\mathrm{C}_{7} \mathrm{H}_{15} \mathrm{ClF}_{3} \mathrm{~N}_{2}{ }^{+}[\mathrm{M}+\mathrm{H}]^{+}$219.0876, found 219.0880.

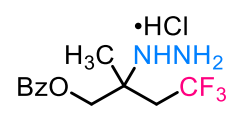

2-Methylallyl benzoate 1t is a known compound and it was prepared according to a literature procedure. ${ }^{[5]}$

4,4,4-Trifluoro-2-hydrazinyl-2-methylbutyl benzoate hydrochloride (4t): compound 4t was prepared from 2-methylallyl benzoate 1t by following the general procedure (foam, $53 \mathrm{mg}$, $83 \%$ yield). ${ }^{1} \mathrm{H}$ NMR $\left(400 \mathrm{MHz}, \mathrm{CD}_{3} \mathrm{OD}\right) \delta 8.22-8.06(\mathrm{~m}, 2 \mathrm{H}), 7.70-7.61(\mathrm{~m}, 1 \mathrm{H}), 7.58-$ $7.47(\mathrm{~m}, 2 \mathrm{H}), 4.51(\mathrm{~d}, J=12.4 \mathrm{~Hz}, 1 \mathrm{H}), 4.42(\mathrm{~d}, J=12.4 \mathrm{~Hz}, 1 \mathrm{H}), 3.00-2.52(\mathrm{~m}, 2 \mathrm{H}), 1.49$ $(\mathrm{s}, 3 \mathrm{H}) ;{ }^{19} \mathrm{~F}$ NMR $\left(376 \mathrm{MHz}, \mathrm{CD}_{3} \mathrm{OD}\right) \delta-61.04(\mathrm{t}, J=11.4 \mathrm{~Hz}) ;{ }^{13} \mathrm{C}\left\{{ }^{1} \mathrm{H}\right\}$ NMR $(101 \mathrm{MHz}$, 
$\left.\mathrm{CD}_{3} \mathrm{OD}\right) \delta 166.1,133.4,129.5,129.1,128.4,125.7(\mathrm{q}, J=270.4 \mathrm{~Hz}), 65.8,57.0,37.0(\mathrm{q}, J=$

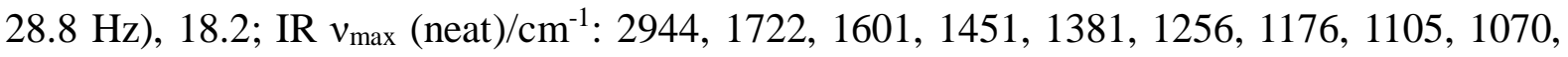
1027, 709; HRMS (ESI, m/z): calcd for $\mathrm{C}_{12} \mathrm{H}_{17} \mathrm{ClF}_{3} \mathrm{~N}_{2} \mathrm{O}_{2}{ }^{+}[\mathrm{M}+\mathrm{H}]^{+} 313.0931$, found 313.0935.

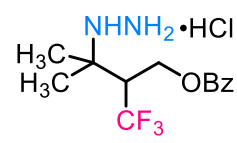

3-Methylbut-2-en-1-yl benzoate $1 \mathbf{u}$ is a known compound and it was prepared according to a literature procedure. ${ }^{[6]}$

3-Hydrazinyl-3-methyl-2-(trifluoromethyl)butyl benzoate hydrochloride (4u): compound $\mathbf{4 u}$ was prepared from 3-methylbut-2-en-1-yl benzoate $\mathbf{1 u}$ by following the general procedure (foam, $51 \mathrm{mg}, 78 \%$ yield). ${ }^{1} \mathrm{H}$ NMR (400 MHz, $\left.\mathrm{CD}_{3} \mathrm{OD}\right) \delta 8.13-7.98(\mathrm{~m}, 2 \mathrm{H}), 7.70-7.58$ $(\mathrm{m}, 1 \mathrm{H}), 7.55-7.44(\mathrm{~m}, 2 \mathrm{H}), 4.82(\mathrm{dd}, J=12.6,3.3 \mathrm{~Hz}, 1 \mathrm{H}), 4.62(\mathrm{dd}, J=12.6,5.5 \mathrm{~Hz}, 1 \mathrm{H})$, $3.38-2.98(\mathrm{~m}, 1 \mathrm{H}), 1.51(\mathrm{~s}, 3 \mathrm{H}), 1.44(\mathrm{~s}, 3 \mathrm{H}) ;{ }^{19} \mathrm{~F} \mathrm{NMR}\left(376 \mathrm{MHz}, \mathrm{CD}_{3} \mathrm{OD}\right) \delta-63.55 ;{ }^{13} \mathrm{C}\left\{{ }^{1} \mathrm{H}\right\}$ NMR (101 MHz, CD $\left.{ }_{3} \mathrm{OD}\right) \delta 165.6,134.1,129.7,129.6,129.3,127.3$ (q, $\left.J=286.8 \mathrm{~Hz}\right), 60.2$, 56.7, 47.0, 24.2, 22.8; IR $v_{\max }$ (neat) $/ \mathrm{cm}^{-1}:$ 2972, 1725, 1646, 1379, 1086, 1044, 879; HRMS (ESI, m/z): calcd for $\mathrm{C}_{13} \mathrm{H}_{19} \mathrm{ClF}_{3} \mathrm{~N}_{2} \mathrm{O}_{2}{ }^{+}[\mathrm{M}+\mathrm{H}]^{+} 327.1087$, found 327.1082 .

\section{Synthetic Applications}
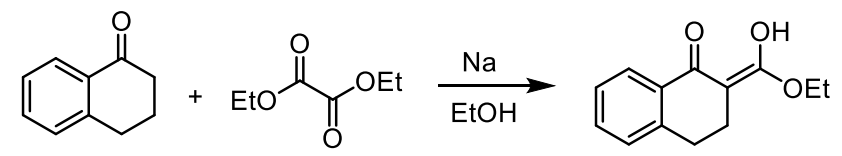

5

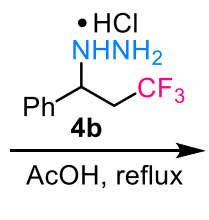

Compound 5 was synthesized according to a literature procedure. ${ }^{[7]}$ Sodium lumps $(276 \mathrm{mg}$, $12 \mathrm{mmol})$ were added in small portions to dry ethanol $(5 \mathrm{ml})$ and stirred until the sodium was completely dissolved. Ethyl oxalate $(813 \mu \mathrm{L}, 6 \mathrm{mmol})$ was added, followed by the dropwise addition of a solution of $\alpha$-tetralone ( $798 \mu \mathrm{L}, 6 \mathrm{mmol}$ in $30 \mathrm{~mL}$ EtOH). The solution was stirred at room temperature for $2 \mathrm{~h}$. The mixture was slowly poured into hydrochloride acid $(2 \mathrm{~N})$ and the resulting precipitate was collected by filtration and washed with a small volume of icecooled ethanol and water to afford product 5 (yellow solid, $1.1 \mathrm{~g}, 84 \%$ yield, m.p. $44-45^{\circ} \mathrm{C}$ ). ${ }^{1} \mathrm{H}$ NMR $\left(400 \mathrm{MHz}, \mathrm{CDCl}_{3}\right) \delta 15.75(\mathrm{~s}, 1 \mathrm{H}), 8.00(\mathrm{~d}, J=7.7,1 \mathrm{H}), 7.53-7.44(\mathrm{~m}, 1 \mathrm{H}), 7.40-$ 
$7.32(\mathrm{~m}, 1 \mathrm{H}), 7.25(\mathrm{~d}, J=7.7 \mathrm{~Hz}, 1 \mathrm{H}), 4.41(\mathrm{q}, J=7.2 \mathrm{~Hz}, 2 \mathrm{H}), 2.98-22.88(\mathrm{~m}, 4 \mathrm{H}), 1.43(\mathrm{t}$, $J=7.1 \mathrm{~Hz}, 3 \mathrm{H})$.

4b was prepared on a larger scale through a slightly modified procedure: To a sealable test tube (inner diameter $=15 \mathrm{~mm}$, length $=100 \mathrm{~mm}$ ) equipped with a stir bar were added ditert-butyl azodicarboxylate $2(372 \mathrm{mg}, 1.6 \mathrm{mmol})$ and $\mathrm{CF}_{3} \mathrm{SO}_{2} \mathrm{Na}(252 \mathrm{mg}, 1.6 \mathrm{mmol})$ and MesAcrMe ${ }^{+} \mathrm{ClO}_{4}{ }^{-}(6.4 \mathrm{mg}, 0.016 \mathrm{mmol})$ in a glovebox. After the vial was taken out of the glovebox, anhydrous $\mathrm{CH}_{2} \mathrm{Cl}_{2}(1.6 \mathrm{~mL}), \mathrm{CH}_{3} \mathrm{CN}(6.4 \mathrm{~mL})$ and styrene $(92 \mu \mathrm{L}, 0.8 \mathrm{mmol})$ were added successively through syringes under $\mathrm{N}_{2}$. The reaction was then stirred vigorously under the irradiation of blue light (6 W LEDs, the photoreactor was maintained at between 35 to 40 ${ }^{\circ} \mathrm{C}$ by circulating water cooling). The reaction process was monitored with TLC. Upon the completion of reaction, two of the above parallel reactions were combined and quenched with saturated $\mathrm{NH}_{4} \mathrm{Cl}$ (aq., $\left.10 \mathrm{~mL}\right)$, extracted with EtOAc $(10 \mathrm{~mL} \times 3)$, dried over anhydrous $\mathrm{Na}_{2} \mathrm{SO}_{4}$ and concentrated in vacuo to afford the crude product, which was then purified through a silica gel flash column to afford a pure product 3. Compound $\mathbf{3}$ was then dissolved in $\mathrm{CH}_{3} \mathrm{OH}$ (12 $\mathrm{mL})$ in a round bottom flask, to which $\mathrm{HCl}\left(4 \mathrm{M}\right.$ in $\left.\mathrm{CH}_{3} \mathrm{OH}, 4 \mathrm{~mL}\right)$ was slowly added at $0{ }^{\circ} \mathrm{C}$. The reaction was then sealed with a PTFE septum and stirred at room temperature until the consumption of compound 3. After the solvent was removed under reduced pressure, the residue was washed with anhydrous ether $(5 \mathrm{~mL} \times 2)$ and then dried in vacuo to afford compound $\mathbf{4 b}$ (269 $\mathrm{mg}, 70 \%$ yield).

A stirred mixture of the $\mathbf{5}(87.3 \mathrm{mg}, 0.4 \mathrm{mmol})$ and the hydrazinium chloride $\mathbf{4 b}(110.5 \mathrm{mg}$, $0.46 \mathrm{mmol})$ in $\mathrm{AcOH}(3 \mathrm{ml})$ was heated under reflux in an oil bath for $2 \mathrm{~h}$. The reaction was allowed to cool to room temperature and carefully quenched with saturated $\mathrm{NaHCO}_{3}$ (aq., 10 $\mathrm{mL})$ and extracted with EtOAc $(5 \mathrm{~mL} \times 3)$. The organic layers were combined and washed with brine, dried over anhydrous $\mathrm{Na}_{2} \mathrm{SO}_{4}$. After being concentrated in vacuo, the crude product was purified through s silica gel flash column to afford pyrazole 6 (yellowish oil, $144 \mathrm{mg}, 87 \%$ yield). ${ }^{1} \mathrm{H}$ NMR $\left(400 \mathrm{MHz}, \mathrm{CDCl}_{3}\right) \delta 7.41-7.34(\mathrm{~m}, 5 \mathrm{H}), 7.34-7.27(\mathrm{~m}, 2 \mathrm{H}), 7.27-7.18(\mathrm{~m}$, 2H), $6.15(\mathrm{dd}, J=9.0,4.1 \mathrm{~Hz}, 1 \mathrm{H}), 4.44$ (q, $J=7.2,2 \mathrm{H}), 3.99-3.82(\mathrm{~m}, 1 \mathrm{H}), 3.15$ (dt, $J=$ $15.3,5.4,1 \mathrm{H}), 3.06-2.73(\mathrm{~m}, 4 \mathrm{H}), 1.43(\mathrm{t}, J=7.2,3 \mathrm{H}),{ }^{19} \mathrm{~F}$ NMR $\left(376 \mathrm{MHz}, \mathrm{CDCl}_{3}\right) \delta-64.93$ $(\mathrm{t}, J=10.4 \mathrm{~Hz}) ;{ }^{13} \mathrm{C}\left\{{ }^{1} \mathrm{H}\right\} \mathrm{NMR}\left(101 \mathrm{MHz}, \mathrm{CDCl}_{3}\right) \delta 162.9,140.3,139.2,138.8,138.2,129.14$, 
129.09, 128.5, 128.1, 127.0, 126.6, 126.2, 125.6 (q, $J=275.7 \mathrm{~Hz}), 122.5,122.4,60.8,58.7$, 40.5 (q, $J=28.4 \mathrm{~Hz}), 30.5,20.2,14.4$; IR $v_{\max }$ (neat) $/ \mathrm{cm}^{-1}: 2980,1715,1436,1376,1176,1213$, 1156, 1098, 1006, 908, 762, 733, 700; HRMS (ESI, m/z): calcd for $\mathrm{C}_{23} \mathrm{H}_{21} \mathrm{~F}_{3} \mathrm{~N}_{2} \mathrm{NaO}_{2}{ }^{+}[\mathrm{M}+$ $\mathrm{Na}]^{+}$437.1447, found 437.1450.

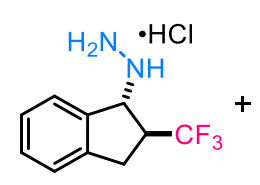

$4 \mathbf{i}$

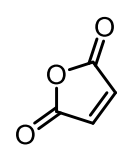

(1.1 equiv)

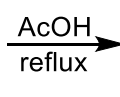

$\underbrace{(1)}_{7} \mathrm{CF}_{3}$

1-(trans-2-Trifluoromethyl)-2,3-dihydro-1H-inden-1-yl)-1,2-dihydropyridazine-3,6-

dione (7): compound 7 was synthesized by following a literature procedure. ${ }^{[8]}$ To a solution of $4 \mathbf{i}(0.2 \mathrm{mmol})$ in $\mathrm{AcOH}(2 \mathrm{~mL})$ was added maleic anhydride $(22 \mathrm{mg}, 0.22 \mathrm{mmol})$. The mixture was heated under reflux in an oil bath for $4 \mathrm{~h}$ until $\mathbf{4 i}$ was consumed (monitored by TLC). The reaction was then cooled down, carefully quenched with saturated $\mathrm{NaHCO}_{3}(\mathrm{aq} ., 10 \mathrm{~mL}$ ) and extracted with EtOAc $(5 \mathrm{~mL} \times 3)$. The organic layers were combined and washed with brine, dried over anhydrous $\mathrm{Na}_{2} \mathrm{SO}_{4}$. After being concentrated in vacuo, the crude product was purified through a silica gel flash column $(\mathrm{PE}: \mathrm{EtOAc}=2: 1)$ to give compound 7 as colorless oil (48 mg, 75\% yield). ${ }^{1} \mathrm{H}$ NMR (600 MHz, DMSO-d6) $\delta 11.28$ (brs, $\left.1 \mathrm{H}\right), 7.35$ (d, $J=7.5 \mathrm{~Hz}$, 1H), $7.33-7.28(\mathrm{~m}, 1 \mathrm{H}), 7.27-7.20(\mathrm{~m}, 1 \mathrm{H}), 7.10(\mathrm{~d}, J=9.8 \mathrm{~Hz}, 1 \mathrm{H}), 7.03(\mathrm{~d}, J=9.8 \mathrm{~Hz}$, 1H), $6.98(\mathrm{~d}, J=7.5 \mathrm{~Hz}, 1 \mathrm{H}), 6.69$ (d, $J=7.7 \mathrm{~Hz}, 1 \mathrm{H}), 3.74$ (hept, $J=9.2 \mathrm{~Hz}, 1 \mathrm{H}), 3.45$ (dd, $J$ $=16.2,9.4 \mathrm{~Hz}, 1 \mathrm{H}), 3.13(\mathrm{dd}, J=16.2,8.4 \mathrm{~Hz}, 1 \mathrm{H}) ;{ }^{19} \mathrm{~F}$ NMR $\left(565 \mathrm{MHz}, \mathrm{DMSO}-d_{6}\right) \delta-69.27$ $(\mathrm{d}, J=9.4 \mathrm{~Hz}) ;{ }^{13} \mathrm{C}\left\{{ }^{1} \mathrm{H}\right\}$ NMR (151 MHz, DMSO-d $\left.d_{6}\right) \delta 158.7,153.5,140.4,140.3,133.1$, 129.0, 128.03 (q, $J=277.5 \mathrm{~Hz}), 128.95,127.9,125.4,124.0,60.6,60.2,46.9$ (d, $J=26.8 \mathrm{~Hz})$,

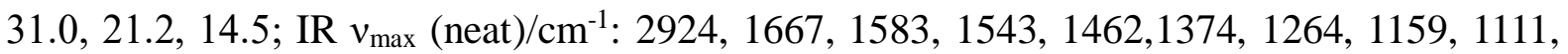
739, 702; HRMS (ESI, m/z): calcd for $\mathrm{C}_{14} \mathrm{H}_{11} \mathrm{~F}_{3} \mathrm{~N}_{2} \mathrm{NaO}_{2}{ }^{+}[\mathrm{M}+\mathrm{Na}]^{+}$319.0665, found 319.0661 .

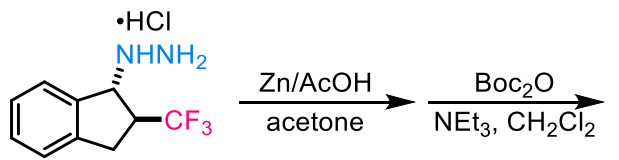

$4 \mathbf{i}$

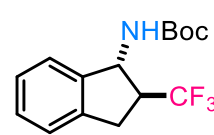

tert-Butyl (2-(trifluoromethyl)-2,3-dihydro-1H-inden-1-yl)carbamate (8): compound 8 was prepared according to a literature procedure. ${ }^{[9]}$ To a solution of $\mathbf{4 i}(0.2 \mathrm{mmol}$, dissolved in $2 \mathrm{~mL}$ of acetone) acetic acid $(3 \mathrm{~mL})$ and freshly activated $\mathrm{Zn}$ dust (130 $\mathrm{mg}, 2 \mathrm{mmol})$ were 
added. After $2 \mathrm{~h}$, the reaction was filtered through Celite and washed with $\mathrm{MeOH}(4 \mathrm{~mL})$. The mixture was acidified with $37 \% \mathrm{HCl}(\mathrm{pH}=1)$, and the solvents were removed under reduced pressure. The residue was then dissolved in $\mathrm{HCl}(1 \mathrm{M}, 20 \mathrm{~mL})$ and washed with $\mathrm{Et}_{2} \mathrm{O}(3 \times 15$ $\mathrm{mL}$ ). The $\mathrm{pH}$ of the aqueous phase was adjusted to 12 with $\mathrm{NaOH}$ pellets and the resulting solution was saturated with $\mathrm{NaCl}$ and extracted with $\mathrm{Et}_{2} \mathrm{O}(3 \times 20 \mathrm{~mL})$. The combined organic layers were dried over $\mathrm{Na}_{2} \mathrm{SO}_{4}$, filtered and concentrated in vacuo to afford the crude amine. The crude product was then dissolved in $\mathrm{CH}_{2} \mathrm{Cl}_{2}, \mathrm{NEt}_{3}(55 \mu \mathrm{L}, 0.4 \mathrm{mmol})$ and $(\mathrm{Boc})_{2} \mathrm{O}(87.3$ $\mathrm{mg}, 0.4 \mathrm{mmol}$ ) was added to the solution and stirred at room temperature for $5 \mathrm{~h}$. Compound $\mathbf{8}$ was then isolated through a silica gel flash column (PE:EtOAc $=20: 1)$ as colorless oil (44 mg, $68 \%$ yield, over 2 steps). ${ }^{1} \mathrm{H}$ NMR (400 MHz, $\left.\mathrm{CDCl}_{3}\right) \delta 7.45-7.06(\mathrm{~m}, 4 \mathrm{H}), 5.57-5.19$ (m, 1H), $5.00-4.59(\mathrm{~m}, 1 \mathrm{H}), 3.21(\mathrm{dd}, J=16.0,8.9 \mathrm{~Hz}, 1 \mathrm{H}), 3.04(\mathrm{dd}, J=16.0,8.7 \mathrm{~Hz}, 1 \mathrm{H}), 2.93$ (hept, $J=8.8 \mathrm{~Hz}, 1 \mathrm{H}), 1.46(\mathrm{~s}, 9 \mathrm{H}) ;{ }^{19} \mathrm{~F}$ NMR $\left(376 \mathrm{MHz}, \mathrm{CDCl}_{3}\right) \delta-70.25(\mathrm{~d}, J=8.7 \mathrm{~Hz})$; ${ }^{13} \mathrm{C}\left\{{ }^{1} \mathrm{H}\right\} \operatorname{NMR}\left(101 \mathrm{MHz}, \mathrm{CDCl}_{3}\right) \delta 155.1,141.3,139.0,128.6,127.5,127.1$ (q, $\left.J=277.1 \mathrm{~Hz}\right)$, 124.7, 124.0, 80.0, 56.1, 51.0 (q, $J=26.6 \mathrm{~Hz}$ ), 30.7, 28.3; IR $v_{\max }$ (neat) $/ \mathrm{cm}^{-1}: 3333,2979$, 1688, 1525, 1393, 1367, 1265, 1158, 1151, 1010, 907, 733; HRMS (ESI, m/z): calcd for $\mathrm{C}_{15} \mathrm{H}_{18} \mathrm{~F}_{3} \mathrm{NNaO}_{2}^{+}[\mathrm{M}+\mathrm{Na}]^{+} 324.1182$, found 324.1185 .

\section{Mechanistic Studies}

\section{i. The reaction with nucleophilic addition product.}

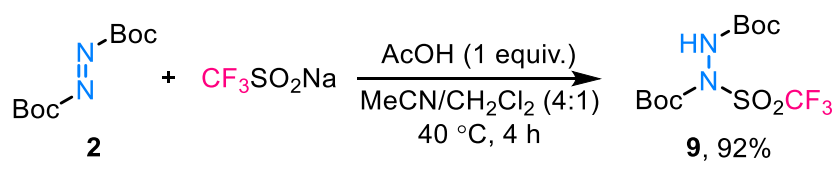

Di-tert-butyl 1-((trifluoromethyl)sulfonyl)hydrazine-1,2-dicarboxylate (9): To a ovendried 2-dram vial charged with a stir bar, di-tert-butyl azodicarboxylate 2 (93 mg, $0.4 \mathrm{mmol}$ ) and $\mathrm{CF}_{3} \mathrm{SO}_{2} \mathrm{Na}(63 \mathrm{mg}, 0.4 \mathrm{mmol})$ were added $\mathrm{CH}_{3} \mathrm{CN}(3.2 \mathrm{~mL}), \mathrm{CH}_{2} \mathrm{Cl}_{2}(0.8 \mathrm{~mL})$ and $\mathrm{AcOH}$ (24 $\mu \mathrm{L}, 0.4 \mathrm{mmol}$ ) successively. The mixture was stirred at $40{ }^{\circ} \mathrm{C}$ in an oil bath for $4 \mathrm{~h}$ and concentrated in vacuo. The nucleophilic adduct 9 was isolated through a silica gel flash column (Hexanes/EtOAc from 20:1 to 10:1) as a white solid (134 mg, 92\% yield). 


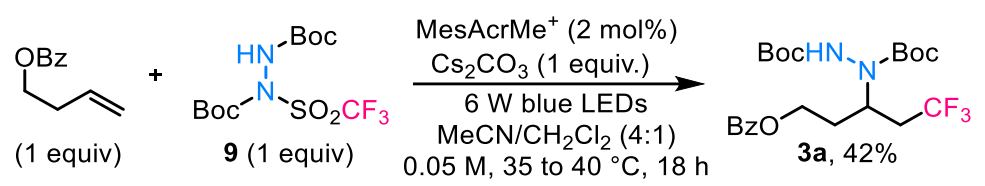

To a sealable 2-dram vial equipped with a stir bar were added $\mathrm{Cs}_{2} \mathrm{CO}_{3}(65 \mathrm{mg}, 0.2 \mathrm{mmol}), 9$ (73 mg, $0.2 \mathrm{mmol})$ and $\mathrm{MesAcrMe}{ }^{+} \mathrm{ClO}_{4}{ }^{-}(1.6 \mathrm{mg}, 0.004 \mathrm{mmol})$ in glovebox. After the vial was taken out of the glovebox, anhydrous $\mathrm{CH}_{2} \mathrm{Cl}_{2}(0.8 \mathrm{~mL}), \mathrm{CH}_{3} \mathrm{CN}(3.2 \mathrm{~mL})$ and alkene $1 \mathrm{a}$ (35 $\mu \mathrm{L}, 0.2 \mathrm{mmol})$ were added to the vial successively through syringes. The reaction was then stirred under the irradiation of blue light (6 W LEDs) (the photoreactor was maintained at between 35 to $40{ }^{\circ} \mathrm{C}$ by circulating water cooling) for $18 \mathrm{~h}$. The resulted mixture was quenched with saturated $\mathrm{NH}_{4} \mathrm{Cl}$ (aq., $2 \mathrm{~mL}$ ), extracted with EtOAc $(2 \mathrm{~mL} \times 3)$, dried over anhydrous $\mathrm{Na}_{2} \mathrm{SO}_{4}$ and concentrated in vacuo. The residue was then purified through a silica gel flash column to afford product 3a (40 mg, $42 \%$ yield).

\section{ii. Competing reactions between a conjugated and an isolated alkene.}

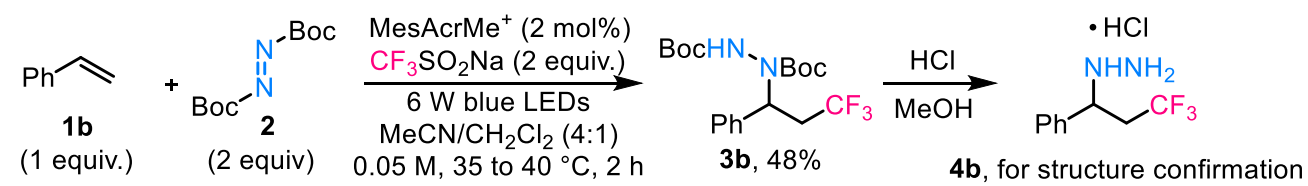

The reaction of styrene $(23 \mu \mathrm{L}, 0.2 \mathrm{mmol})$ was performed by following the general procedure but quenched after 2 h. 3 b was isolated through a silica gel flash column (39 mg, $48 \%$ yield). The purity of product $\mathbf{3 b}$ was confirmed by NMR after deprotection.

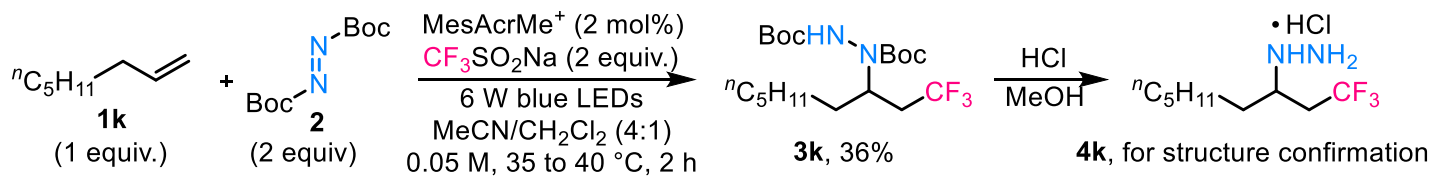

The reaction of 1-octene ( $32 \mu \mathrm{L}, 0.2 \mathrm{mmol}$ ) was also performed by following the general procedure but quenched after $3 \mathrm{~h}$. 3kwas then isolated through a silica gel flash column (25 $\mathrm{mg}, 36 \%$ yield). The purity of product $\mathbf{3 k}$ was confirmed by NMR after deprotection.

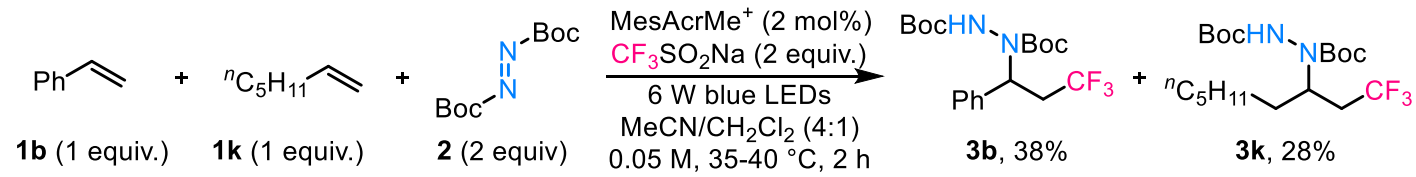

Styrene $(23 \mu \mathrm{L}, 0.2 \mathrm{mmol})$ and 1-octene $(32 \mu \mathrm{L}, 0.2 \mathrm{mmol})$ were subjected to the same reaction 
by following otherwise the same procedure. When the reaction was quenched after $2 \mathrm{~h}, \mathbf{3 b}$ and 3k were isolated through a silica gel flash column ( $31 \mathrm{mg}, 38 \%$ for $\mathbf{3 b}$ and $19 \mathrm{mg}, 28 \%$ for $\mathbf{3 k}$ ), respectively.

\section{iii. Evaluating the reversibility of trifluoromethyl radical addition.}

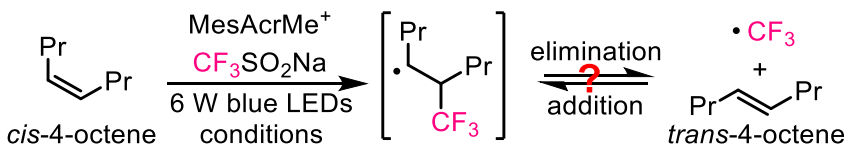

cis-4-Octene is commercially available and it was distilled before use.

To a sealable 2-dram vial equipped with a stir bar were added $\mathrm{CF}_{3} \mathrm{SO}_{2} \mathrm{Na}(62 \mathrm{mg}, 0.4 \mathrm{mmol})$ and $\mathrm{MesAcrMe}^{+}(1.6 \mathrm{mg}, 0.004 \mathrm{mmol})$ in a glovebox. After the vial was taken out of the glovebox, anhydrous $\mathrm{CH}_{2} \mathrm{Cl}_{2}(0.8 \mathrm{~mL})$ and $\mathrm{CH}_{3} \mathrm{CN}(3.2 \mathrm{~mL})$ were added. Then, cis-4-octene (32 $\mu \mathrm{L}, 0.2 \mathrm{mmol})$ and dodecane $(10 \mu \mathrm{L}, 0.044 \mathrm{mmol}$, as an internal standard)were added to the solution successively through syringes. The reaction was then stirred under the irradiation of blue light (6 W LEDs, the photoreactor was maintained at between 35 to $40{ }^{\circ} \mathrm{C}$ by circulating water cooling). Aliquots $(20 \mu \mathrm{L})$ were taken at 30, 60, 90 and $120 \mathrm{~min}$. The samples were diluted with $\mathrm{CH}_{2} \mathrm{Cl}_{2}(0.5 \mathrm{~mL})$, filtered through a Nylon 66 syringe filter and submitted for gas chromatography analysis. The recovery yields of cis- (red dots) and trans-4-octene (black dots) were summarized below. The other set of control experiments was performed accordingly but in the absence of $\mathrm{CF}_{3} \mathrm{SO}_{2} \mathrm{Na}$.

Based on the data obtained, the generation of small amount of trans-4-octene could be ascribed to the photosensitizer and light irradiation. The presence of trifluoromethyl radical gradually consumed alkenes, but did not expedite the cis-alkene isomerization process.

a) reaction with $\mathrm{CF}_{3} \mathrm{SO}_{2} \mathrm{Na}$

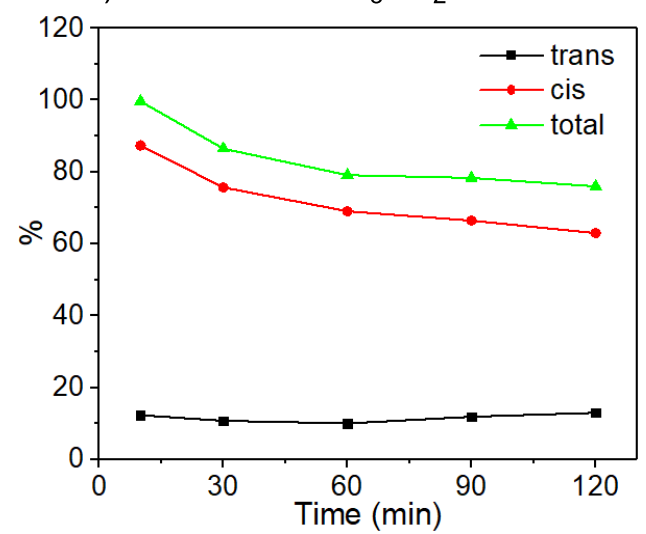

b) reaction without $\mathrm{CF}_{3} \mathrm{SO}_{2} \mathrm{Na}$

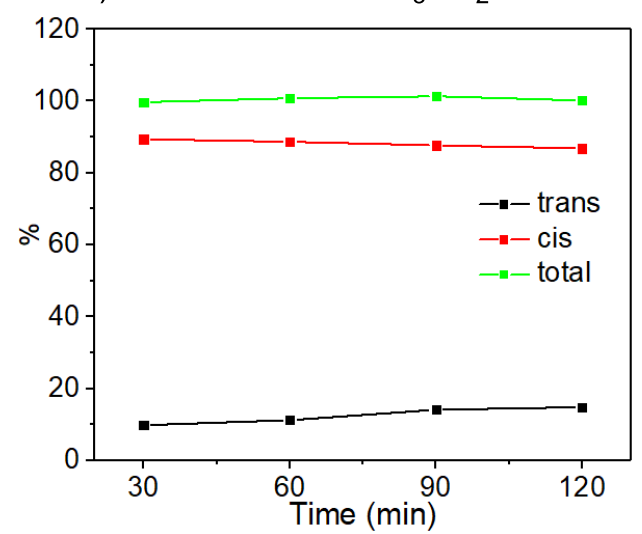




\section{iv. Probing for the rate-determining step.}

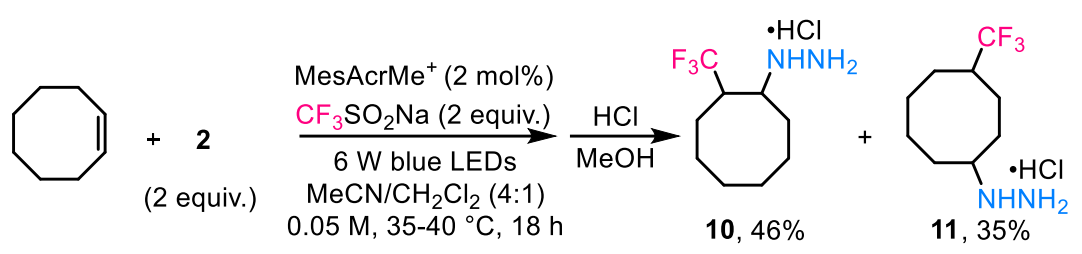

Cyclooctene is commercially available and it was distilled before use.

To a sealable 2-dram vial equipped with a stir bar were added di-tert-butyl azodicarboxylate 2 (93 mg, $0.4 \mathrm{mmol})$ and $\mathrm{CF}_{3} \mathrm{SO}_{2} \mathrm{Na}(62 \mathrm{mg}, 0.4 \mathrm{mmol})$ and $\mathrm{MesAcrMe}^{+} \mathrm{ClO}_{4}{ }^{-}(1.6 \mathrm{mg}, 0.004$ mmol) in glovebox. After the vial was taken out of the glovebox, anhydrous $\mathrm{CH}_{2} \mathrm{Cl}_{2}(0.8 \mathrm{~mL})$, $\mathrm{CH}_{3} \mathrm{CN}(3.2 \mathrm{~mL})$ and cyclooctene $(26 \mu \mathrm{L}, 0.2 \mathrm{mmol})$ were added successively through syringes under $\mathrm{N}_{2}$. The reaction was then stirred under the irradiation of blue light (6 W LEDs, the photoreactor was maintained at between 35 to $40{ }^{\circ} \mathrm{C}$ by circulating water cooling). The reaction process was monitored with TLC. Upon the completion of reaction, the resulted mixture was quenched with saturated $\mathrm{NH}_{4} \mathrm{Cl}$ (aq., $\left.2 \mathrm{~mL}\right)$, extratced with EtOAc $(2 \mathrm{~mL} \times 3)$, dried over anhydrous $\mathrm{Na}_{2} \mathrm{SO}_{4}$ and concentrated in vacuo. The residue was then purified through a silica gel flash column to afford 2 major products, which were then subjected for deprotection with $\mathrm{HCl}$ in $\mathrm{MeOH}$ (same as the general procedure). After the removal of the solvent, hydrazinium chlorides 10 (foam, $23 \mathrm{mg}, 46 \%$ yield) and 11 (foam, $18 \mathrm{mg}, 35 \%$ yield) were obtained, respectively.

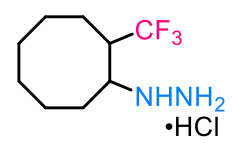

(2-(Trifluoromethyl)cyclooctyl)hydrazine hydrochloride (10): Compound 10 was derived from the less polar isomer and confirmed as a single diastereomer of 1,2-difunctionalization product by ${ }^{1} \mathrm{H}-{ }^{1} \mathrm{H}$ COSY analysis. ${ }^{1} \mathrm{H}$ NMR $\left(400 \mathrm{MHz}, \mathrm{D}_{2} \mathrm{O}\right) \delta 3.59-3.39(\mathrm{~m}, 1 \mathrm{H}), 2.58-$ $2.33(\mathrm{~m}, 1 \mathrm{H}), 1.98-1.76(\mathrm{~m}, 4.3 \mathrm{~Hz}, 3 \mathrm{H}), 1.75-1.49(\mathrm{~m}, 5 \mathrm{H}), 1.46-1.29(\mathrm{~m}, 2 \mathrm{H}), 1.25(\mathrm{~d}$, $J=9.7 \mathrm{~Hz}, 2 \mathrm{H}) ;{ }^{19} \mathrm{~F}$ NMR $\left(376 \mathrm{MHz}, \mathrm{D}_{2} \mathrm{O}\right) \delta-68.31 ;{ }^{13} \mathrm{C}\left\{{ }^{1} \mathrm{H}\right\} \mathrm{NMR}\left(101 \mathrm{MHz}, \mathrm{CD}_{3} \mathrm{OD}\right) \delta$ $128.2(\mathrm{q}, J=280.3 \mathrm{~Hz}), 57.0,44.8(\mathrm{q}, J=23.4 \mathrm{~Hz}), 27.1,25.7,25.3,25.2,23.8,22.6(\mathrm{q}, J=$ $2.8 \mathrm{~Hz}$ ); IR $v_{\max }($ neat $) / \mathrm{cm}^{-1}: 2925,2855,1731,1601,1507,1373,1255,1145,742$; HRMS (ESI, m/z): calcd for $\mathrm{C}_{9} \mathrm{H}_{19} \mathrm{ClF}_{3} \mathrm{~N}_{2}{ }^{+}[\mathrm{M}+\mathrm{H}]^{+} 247.1189$, found 247.1185. 


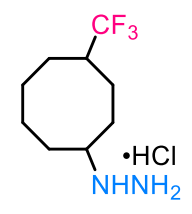

(4-(Trifluoromethyl)cyclooctyl)hydrazine hydrochloride (11): Compound 11 was derived from the more polar isomer as a pair of diastereomers (2:1). ${ }^{1} \mathrm{H} \mathrm{NMR}\left(400 \mathrm{MHz}, \mathrm{D}_{2} \mathrm{O}\right) \delta 3.28$ -3.19 (m, 1H, major), $3.19-3.10$ (m, 1H, minor), $2.35-2.06(\mathrm{~m}, 1 \mathrm{H}), 2.02-1.68(\mathrm{~m}, 4 \mathrm{H})$, $1.68-1.18(\mathrm{~m}, 8 \mathrm{H}) ;{ }^{19} \mathrm{~F}$ NMR $\left(376 \mathrm{MHz}, \mathrm{D}_{2} \mathrm{O}\right) \delta-72.92(\mathrm{~d}, J=10.1 \mathrm{~Hz}) ;{ }^{13} \mathrm{C}\left\{{ }^{1} \mathrm{H}\right\} \mathrm{NMR}(101$ $\left.\mathrm{MHz}, \mathrm{D}_{2} \mathrm{O}\right) \delta 128.67$ (q, $J=279.2 \mathrm{~Hz}$ ) (minor), 128.64 (q, $J=279.2 \mathrm{~Hz}$ ) (major), 61.8 (major), 61.6 (minor), 41.2 (q, $J=24.5 \mathrm{~Hz}$ ) (major), $40.6(\mathrm{q}, J=24.4 \mathrm{~Hz}$ ) (minor), 27.7 (major), 27.0 (minor), 26.2 (major), 24.7 (minor), 24.5 (major + minor), 24.0 (major), 23.8 (minor), 23.3 (major), 23.0 (minor), 22.5 (q, $J=2.3 \mathrm{~Hz}$ ) (major), 21.2 (q, $J=2.3 \mathrm{~Hz}$ ) (minor); IR $v_{\max }$ (neat)/cm ${ }^{-1}:$ 2930, 2858, 1601, 1471, 1396, 1340, 1266, 1164, 1137, 1115, 740; HRMS (ESI, $\mathrm{m} / \mathrm{z}$ ): calcd for $\mathrm{C}_{9} \mathrm{H}_{19} \mathrm{ClF}_{3} \mathrm{~N}_{2}{ }^{+}[\mathrm{M}+\mathrm{H}]^{+} 247.1189$, found 247.1186 .

The ${ }^{1} \mathrm{H}$ and ${ }^{13} \mathrm{C}$ NMR chemical shift of the major isomer was assigned by using ${ }^{1} \mathrm{H}-{ }^{1} \mathrm{H}$ COSY and HSQC, and the values are depicted as follows
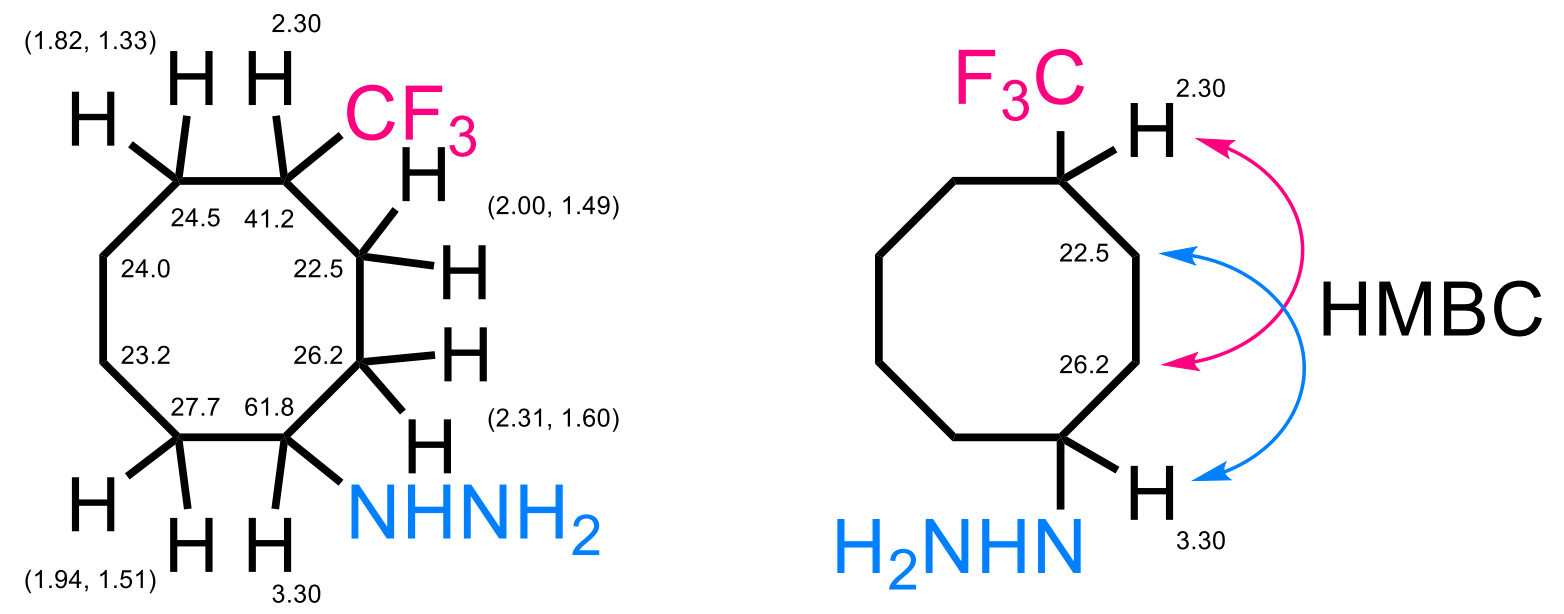

The major isomer was then identified as a 1,4-trifluoromethyl-hydrazination product by HMBC analysis. In addition, the minor isomer follows the same pattern on HMBC, so, the two isomers are highly likely a pair of diastereomers. 


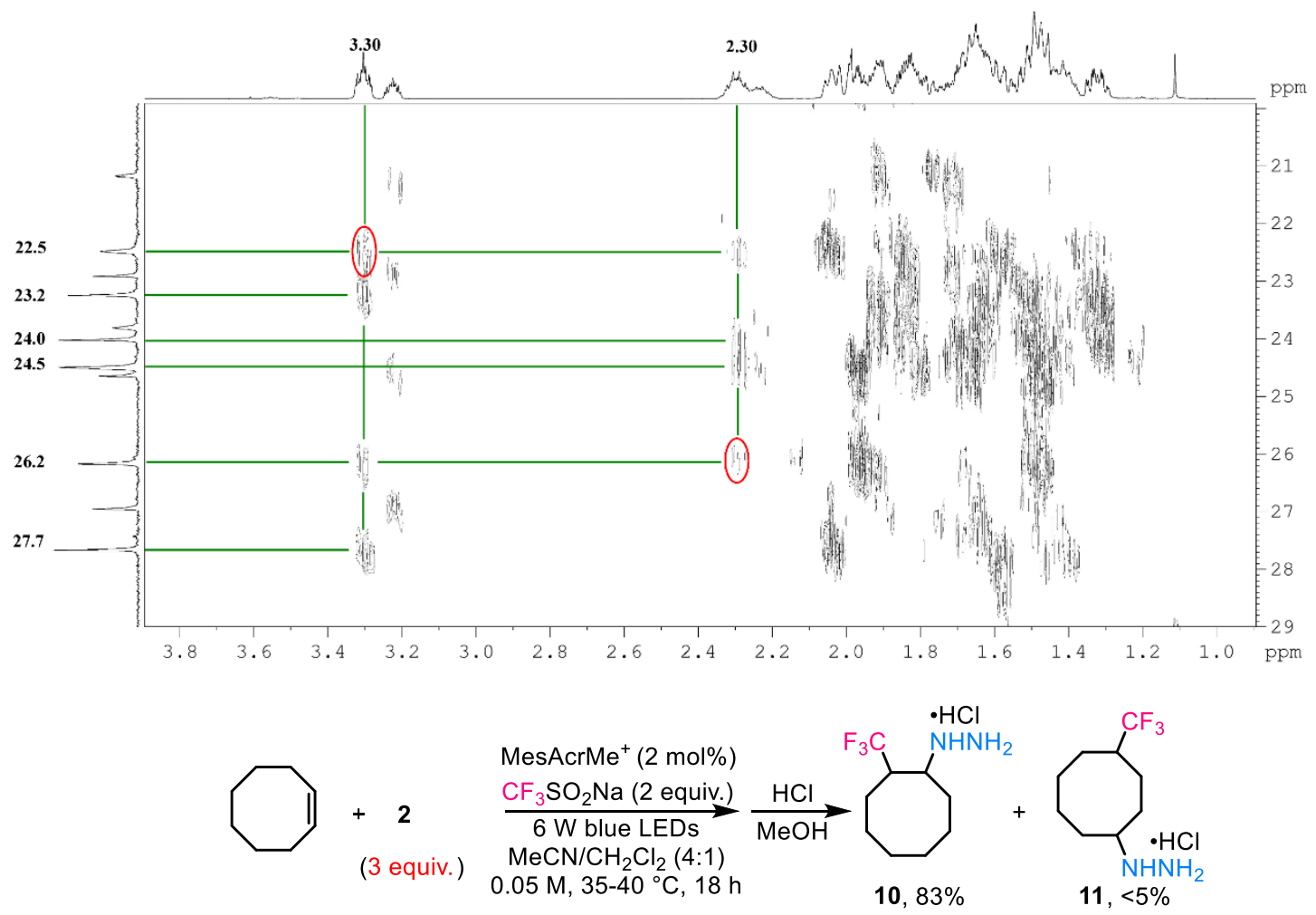

As a consequence, when the amount of BocN=NBoc was increased to 3 equiv. ( 0.6 mmol), the selectivity towards 1,2-trifluoro-hydrazination was significantly improved, affording $\mathbf{1 0}$ in $83 \%$ yield $(41 \mathrm{mg})$ after deprotection.

\section{v. The reaction of 1,5 -cyclooctadiene.}

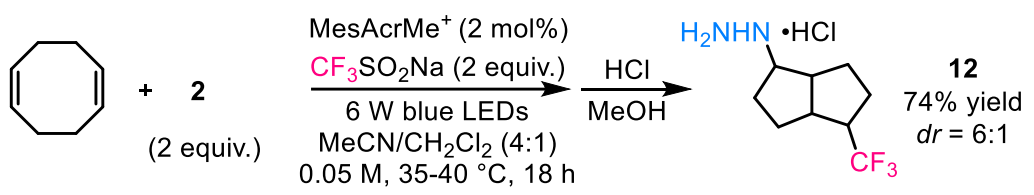

1,5-cyclooctadiene is commercially available and it was distilled before use.

(4-(Trifluoromethyl)octahydropentalen-1-yl)hydrazine (12): The reaction of 1,5cyclooctadiene $(25 \mu \mathrm{L}, 0.2 \mathrm{mmol})$ was performed by following the general procedure. After deprotection, two diastereomers of a bicyclic hydrazinium salt 12 were afforded in $63 \%$ (31 $\mathrm{mg}$ ) and $11 \%(5 \mathrm{mg})$ yields, respectively.

Major isomer: (foam, $31 \mathrm{mg}, 63 \%$ yield ), ${ }^{1} \mathrm{H} \mathrm{NMR}\left(400 \mathrm{MHz}, \mathrm{D}_{2} \mathrm{O}\right) \delta 3.32-3.08(\mathrm{~m}, 1 \mathrm{H})$, $2.71-2.53(\mathrm{~m}, 1 \mathrm{H}), 2.53-2.44(\mathrm{~m}, 1 \mathrm{H}), 2.40-2.21(\mathrm{~m}, 1 \mathrm{H}), 2.04-1.90(\mathrm{~m}, 2 \mathrm{H}), 1.90-1.74$ $(\mathrm{m}, 2 \mathrm{H}), 1.76-1.60(\mathrm{~m}, 1 \mathrm{H}), 1.62-1.50(\mathrm{~m}, 1 \mathrm{H}), 1.50-1.36(\mathrm{~m}, 1 \mathrm{H}), 1.36-1.22(\mathrm{~m}, 1 \mathrm{H})$; ${ }^{19} \mathrm{~F}$ NMR $\left(376 \mathrm{MHz}, \mathrm{D}_{2} \mathrm{O}\right) \delta-70.53(\mathrm{~d}, J=9.5 \mathrm{~Hz}) ;{ }^{13} \mathrm{C}\left\{{ }^{1} \mathrm{H}\right\}$ NMR $\left(101 \mathrm{MHz}, \mathrm{D}_{2} \mathrm{O}\right) \delta 128.3$ 
$(\mathrm{q}, J=277.2 \mathrm{~Hz}), 68.4,49.1(\mathrm{q}, J=26.1 \mathrm{~Hz}), 46.2,43.2,30.7,29.5,28.5,27.2 ; \mathrm{IR} v_{\max }$ (neat) $/ \mathrm{cm}^{-1}:$ 2973, 1682, 1517, 1395, 1316, 1267, 1158, 1103, 1088, 1047, 734, 701; HRMS (ESI, m/z): calcd for $\mathrm{C}_{9} \mathrm{H}_{17} \mathrm{ClF}_{3} \mathrm{~N}_{2}{ }^{+}[\mathrm{M}+\mathrm{H}]^{+}$245.1032, found 245.1037.

Minor isomer: (foam, $5 \mathrm{mg}, 11 \%$ yield), ${ }^{1} \mathrm{H} \mathrm{NMR}\left(400 \mathrm{MHz}, \mathrm{D}_{2} \mathrm{O}\right) \delta 3.58-3.45(\mathrm{~m}, 1 \mathrm{H})$, $2.89-2.73(\mathrm{~m}, 1 \mathrm{H}), 2.62-2.53(\mathrm{~m}, 1 \mathrm{H}), 2.45-2.28(\mathrm{~m}, 1 \mathrm{H}), 2.16-2.06(\mathrm{~m}, 1 \mathrm{H}), 2.04-1.76$ $(\mathrm{m}, 4 \mathrm{H}), 1.72-1.57(\mathrm{~m}, 1 \mathrm{H}), 1.41-1.34(\mathrm{~m}, 2 \mathrm{H}) ;{ }^{19} \mathrm{~F}$ NMR $\left(376 \mathrm{MHz}, \mathrm{D}_{2} \mathrm{O}\right) \delta-70.58(\mathrm{~d}, J=$ $10.0 \mathrm{~Hz}) ;{ }^{13} \mathrm{C}\left\{{ }^{1} \mathrm{H}\right\} \operatorname{NMR}\left(101 \mathrm{MHz}, \mathrm{D}_{2} \mathrm{O}\right) \delta 128.2(\mathrm{q}, J=277.2 \mathrm{~Hz}), 68.4,49.1(\mathrm{q}, J=26.1$ Hz), 46.3, 43.2 , 30.7, 29.5, 28.5, 27.2; IR $v_{\max }$ (neat) $/ \mathrm{cm}^{-1}: 2966,1682,1516,1395,1317,1264$, 1154, 1104, 735, 701; HRMS (ESI, m/z): calcd for $\mathrm{C}_{9} \mathrm{H}_{17} \mathrm{ClF}_{3} \mathrm{~N}_{2}{ }^{+}[\mathrm{M}+\mathrm{H}]^{+}$245.1032, found 245.1030.

\section{vi. The reaction of $\beta$-pinene.}

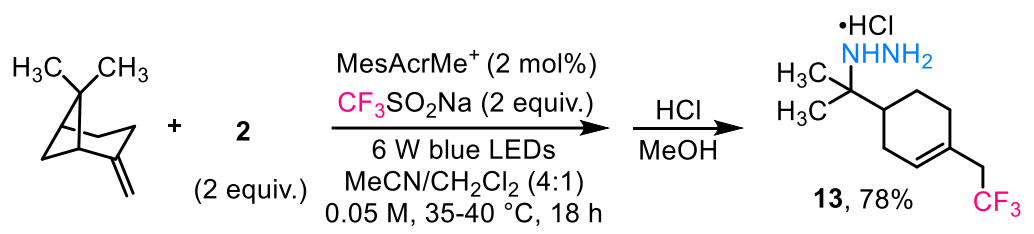

$\beta$-pinene is commercially available and it was distilled before use.

(2-(4-(2,2,2-Trifluoroethyl)cyclohex-3-en-1-yl)propan-2-yl)hydrazine hydrochloride (13): The reaction of $\beta$-pinene ( $31 \mu \mathrm{L}, 0.2 \mathrm{mmol}$ ) was performed by following the general procedure. A ring-openning compound 13 was obtained after deprotection (foam, $43 \mathrm{mg}, 78 \%$ yield). ${ }^{1} \mathrm{H}$ NMR (400 MHz, CD 3 OD) $\delta 5.79-5.71(\mathrm{~m}, 1 \mathrm{H}), 2.85(\mathrm{q}, J=11.4 \mathrm{~Hz}, 2 \mathrm{H}), 2.33-2.09$ (m, $3 \mathrm{H}), 2.06-1.86(\mathrm{~m}, 3 \mathrm{H}), 1.38-1.34(\mathrm{~m}, 1 \mathrm{H}), 1.33(\mathrm{~s}, 3 \mathrm{H}), 1.31(\mathrm{~s}, 3 \mathrm{H}) ;{ }^{19} \mathrm{~F}$ NMR $(376 \mathrm{MHz}$, $\left.\mathrm{CD}_{3} \mathrm{OD}\right) \delta-67.82(\mathrm{t}, J=11.3 \mathrm{~Hz}) ;{ }^{13} \mathrm{C}\left\{{ }^{1} \mathrm{H}\right\} \mathrm{NMR}\left(101 \mathrm{MHz}, \mathrm{CD}_{3} \mathrm{OD}\right) \delta 127.8,127.2,126.4$ $(\mathrm{q}, J=278.2 \mathrm{~Hz}), 63.6,40.7(\mathrm{q}, J=28.8 \mathrm{~Hz}), 38.1,28.8,25.9,23.1,18.8,18.7$; IR $v_{\max }$ (neat)/cm ${ }^{-1}:$ 2978, 1703, 1454, 1365, 1256, 1160, 1129, 1083, 737; HRMS (ESI, m/z): calcd for $\mathrm{C}_{11} \mathrm{H}_{21} \mathrm{ClF}_{3} \mathrm{~N}_{2}{ }^{+}[\mathrm{M}+\mathrm{H}]^{+}$273.1345, found 273.1350.

\section{vi. Allylic hydrazination via a radical chain process.}

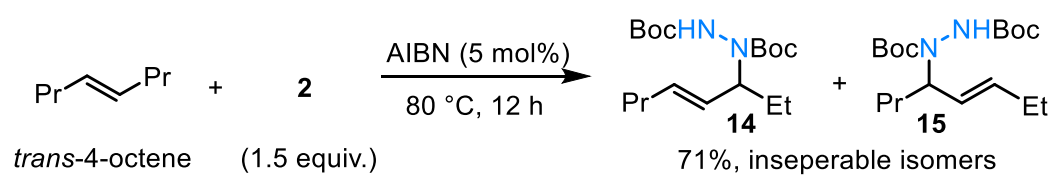


To a sealable tube equipped with a stir bar were added di-tert-butyl azodicarboxylate 2 (70 mg, $0.3 \mathrm{mmol})$ and AIBN (1.7 mg, $0.01 \mathrm{mmol})$. After the vial was evacuated and backfilled with $\mathrm{N}_{2}$ three times, anhydrous $\mathrm{CH}_{2} \mathrm{Cl}_{2}(1.6 \mathrm{~mL})$ and $\mathrm{CH}_{3} \mathrm{CN}(0.4 \mathrm{~mL})$ and trans-4-octene $(31 \mu \mathrm{L}$, $0.2 \mathrm{mmol}$ ) were added successively through syringes. The reaction was then stirred at $80{ }^{\circ} \mathrm{C}$ in an oil bath for $12 \mathrm{~h}$. After the completion of the reaction, the resulted mixture was concentrated in vacuo to afford the crude product, which was then purified through a silica gel flash column to afford products $\mathbf{1 4}$ and $\mathbf{1 5}$ as a pair of inseparable isomers.

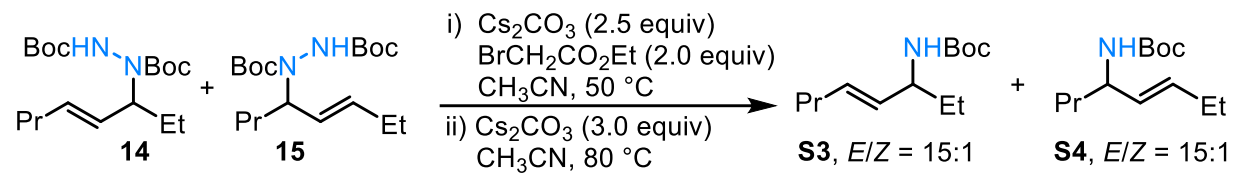

Compounds 14 and 15 were identified after $\mathrm{N}-\mathrm{N}$ cleavage according to a literature procedure, ${ }^{[10]}$ and the characterization data of $\mathbf{S 3}$ and $\mathbf{S 4}$ are in accordance with the literature. ${ }^{1} \mathrm{H}$ NMR $\left(600 \mathrm{MHz}, \mathrm{CDCl}_{3}\right) \delta 5.70-5.53(\mathrm{~m}, 1 \mathrm{H}), 5.32$ (dd, $\left.J=15.4,6.4 \mathrm{~Hz}, 1 \mathrm{H}\right), 4.43$ (brs, 1H), 4.15 $-3.70(\mathrm{~m}, 1 \mathrm{H}), 2.10-1.92(\mathrm{~m}, 2 \mathrm{H}), 1.58-1.48(\mathrm{~m}, 2 \mathrm{H}), 1.46(\mathrm{~s}, 9 \mathrm{H}), 1.43-1.31(\mathrm{~m}, 2 \mathrm{H})$, $0.99(\mathrm{t}, J=7.4 \mathrm{~Hz}, 1.5 \mathrm{H}), 0.96-0.87(\mathrm{~m}, 4.5 \mathrm{H})$.

\section{vii. Side product analysis in catalytic reaction and chain reaction.}

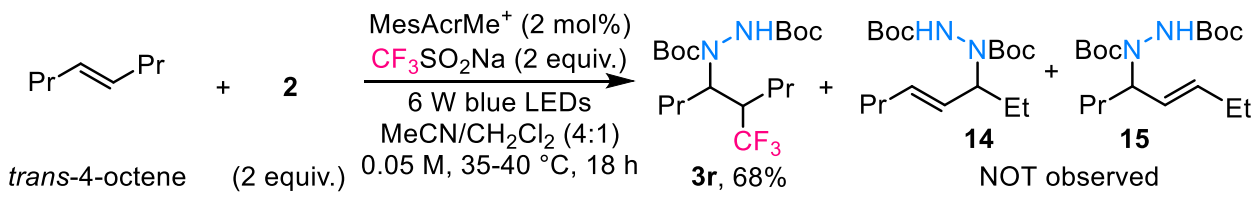

Compound $3 \mathbf{r}$ was obtained from the reaction of trans-4-octene $(31 \mu \mathrm{L}, 0.2 \mathrm{mmol})$ under the standard conditions (56 mg, 68\% yield), while 14 and 15 were not observed.

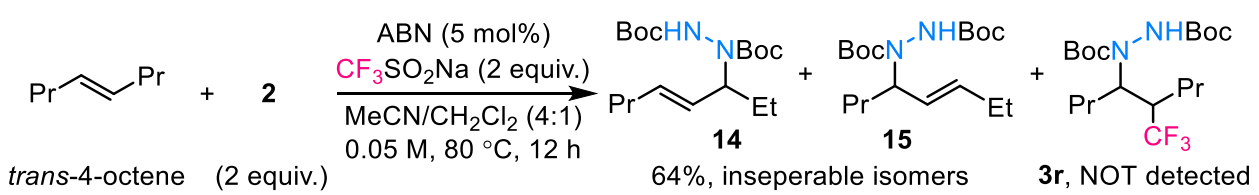

To a sealable 2-dram vial equipped with a stir bar were added di-tert-butyl azodicarboxylate 2 (93 mg, $0.4 \mathrm{mmol}), \mathrm{CF}_{3} \mathrm{SO}_{2} \mathrm{Na}(64 \mathrm{mg}, 0.4 \mathrm{mmol})$ and AIBN (1.7 mg, $\left.0.01 \mathrm{mmol}\right)$. After the vial was evacuated and backfilled with $\mathrm{N}_{2}$ three times, anhydrous $\mathrm{CH}_{2} \mathrm{Cl}_{2}(1.6 \mathrm{~mL})$ and $\mathrm{CH}_{3} \mathrm{CN}$ $(0.4 \mathrm{~mL})$ and trans-4-octene $(31 \mu \mathrm{L}, 0.2 \mathrm{mmol})$ were added successively through syringes. The reaction was then stirred at $80{ }^{\circ} \mathrm{C}$ in an oil bath for $12 \mathrm{~h}$. After the completion of the reaction, 
allylic hydrazination products $\mathbf{1 4}$ and $\mathbf{1 5}$ were generated as the major product without the detection of $\mathbf{3 r}$.

\section{References}

[1] K.-F. Hu, X.-S. Ning, J.-P. Qu, Y.-B. Kang, J. Org. Chem. 2018, 83, 11327.

[2] A. V. Brethomé, R. S. Paton, S. P. Fletcher, ACS Catal. 2019, 9, 7179.

[3] J. Melendez Matos, S. Vásquez-Céspedes, J. Gu, T. Oguma, R. Shenvi, J. Am. Chem. Soc. 2018, 140, 16976.

[4] P. Xu, F. Wang, G. Fan, X. Xu, P. Tang, Angew. Chem. Int. Ed. 2017, 56, 1101.

[5] X. Bertrand, J.-F. Paquin, Org. Lett. 2019, 21, 9759.

[6] J. D. Moore, R. J. Byrne, P. Vedantham, D. L. Flynn, P. R. Hanson, Org. Lett. 2003, 5, 4241.

[7] G. A. Pinna, M. A. Pirisi, J. M. Mussinu, IL Farmaco. 2003, 58, 749.

[8] S. Mayakrishnan, Y. Arun, C. Balachandran, N. Emi, D. Muralidharana, P. T. Perumal, Org. Biomol. Chem. 2016, 14, 1958.

[9] J. Waser, B. Gasper, H. Nambu, E. M. Carreira, J. Am. Chem. Soc. 2006, 128, 11693.

[10] P. Wang, Y. X. Luo, S. S. Zhu, D. F. Lu, Y. F. Gong, Adv. Synth. Catal., 2019, 361, 5565. 


\section{VII. ${ }^{1} \mathrm{H},{ }^{13} \mathrm{C}$ and ${ }^{19} \mathrm{~F}$ NMR Spectra of New Compounds}

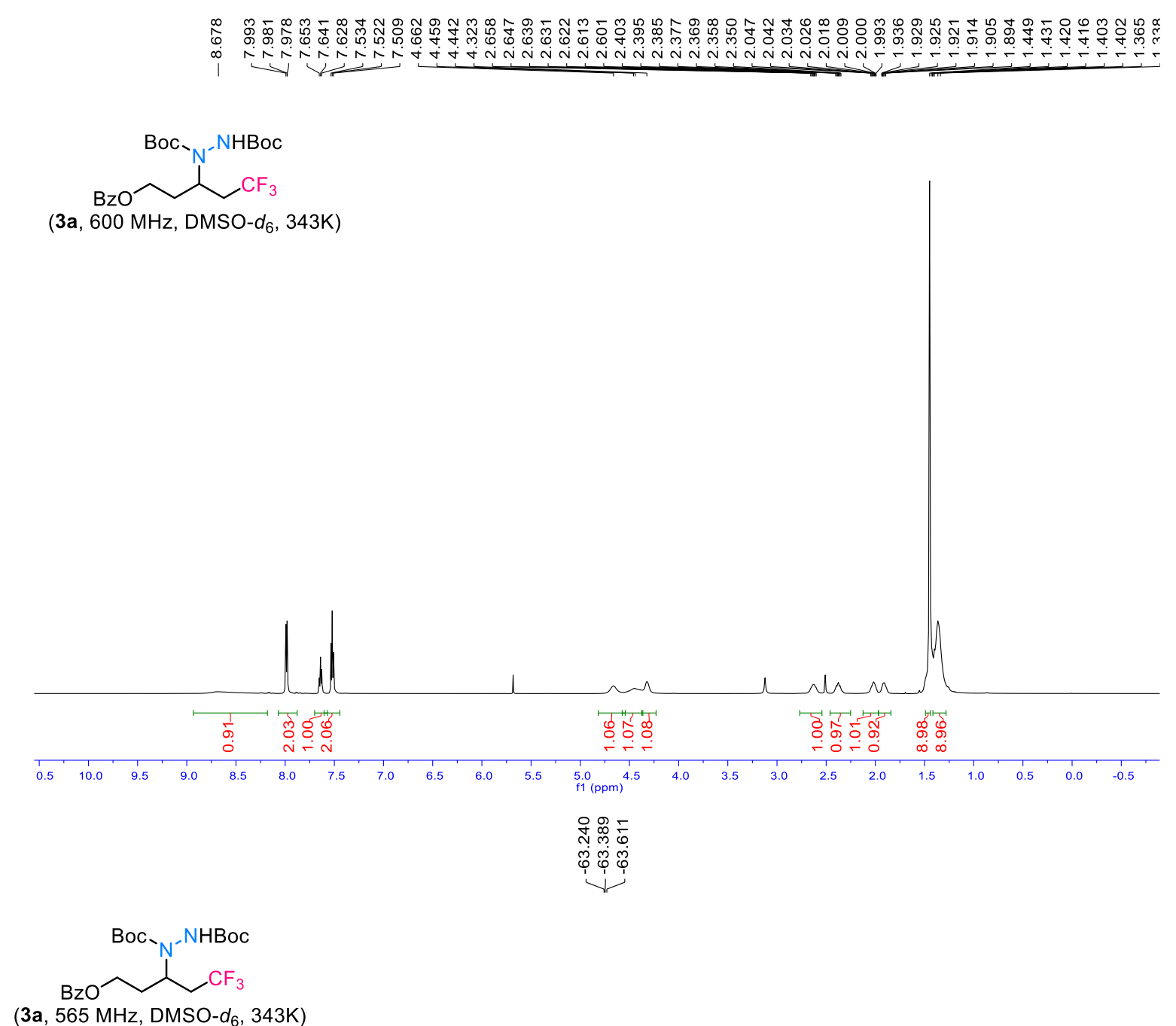

(3a, $565 \mathrm{MHz}, \mathrm{DMSO}-d_{6}, 343 \mathrm{~K}$ )

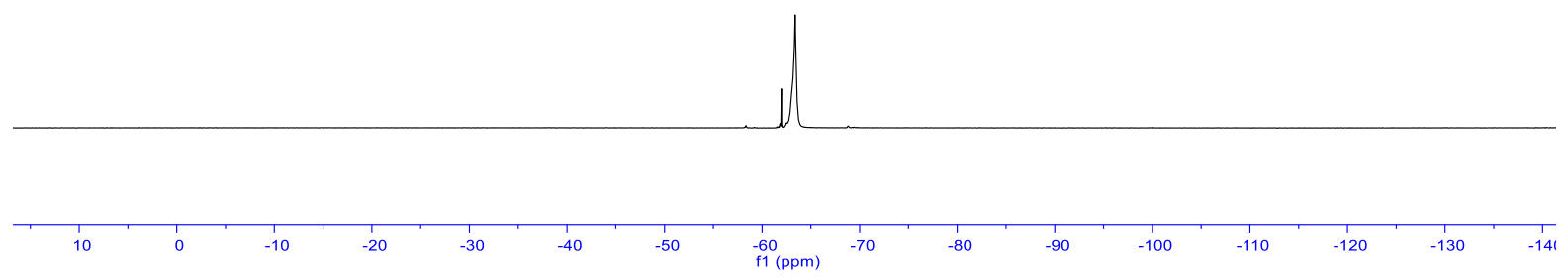



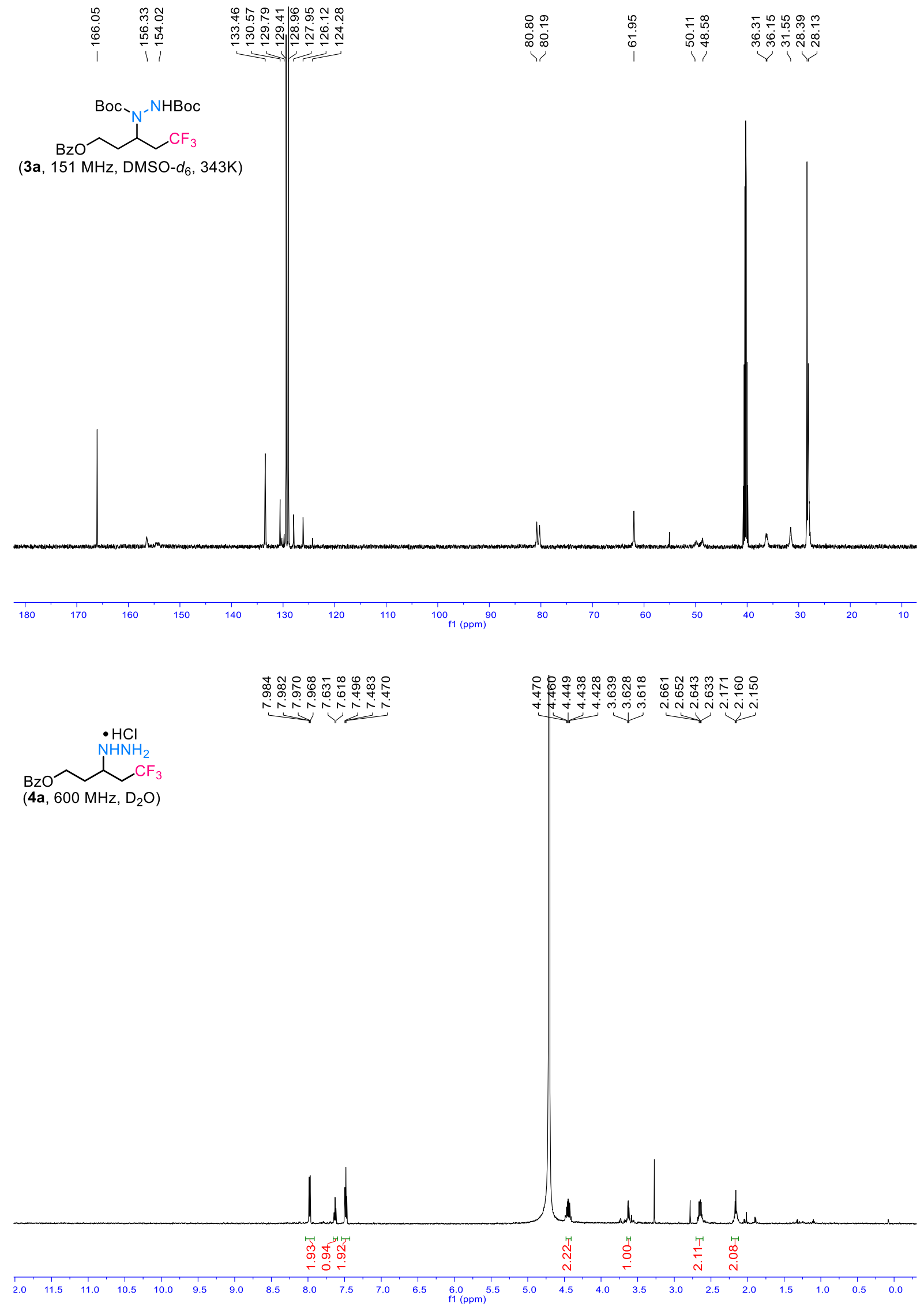
鱼兽兽

象

- $\mathrm{HCl}$

$\mathrm{NHNH}_{2}$

$\mathrm{BzO}_{\left(4 \mathrm{O}, \mathrm{CF}_{3}\right.}$

(4a, $376 \mathrm{MHz}, \mathrm{D}_{2} \mathrm{O}$ )

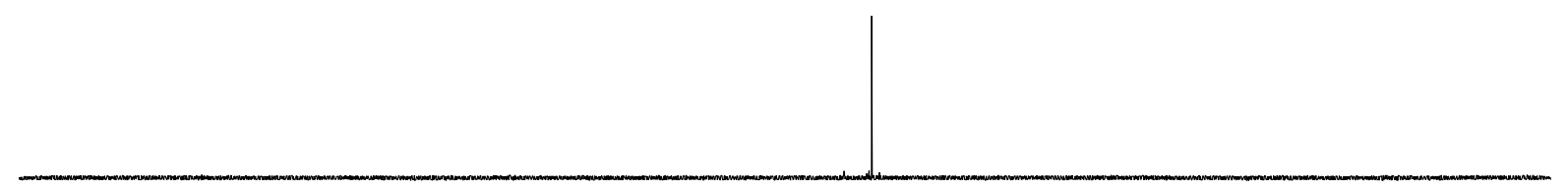

\begin{tabular}{|c|c|c|c|c|c|c|c|c|c|c|c|c|c|c|c|c|c|c|c|c|c|c|}
\hline 60 & 50 & 40 & 30 & 20 & 10 & 0 & -10 & -20 & -30 & -40 & $\begin{array}{c}-50 \\
\mathrm{f} 1(\mathrm{ppm})\end{array}$ & -60 & -70 & -80 & -90 & -100 & -110 & $\begin{array}{c}1 \\
-120\end{array}$ & -130 & -140 & -150 & -160 \\
\hline & & & & & & & 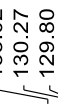 & 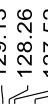 & 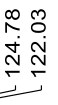 & & & & & & & & 离 & 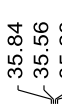 & 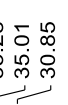 & & & \\
\hline
\end{tabular}

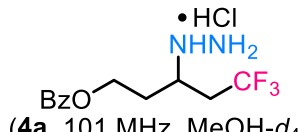

(4a, $101 \mathrm{MHz}, \mathrm{MeOH}-d_{4}$ )
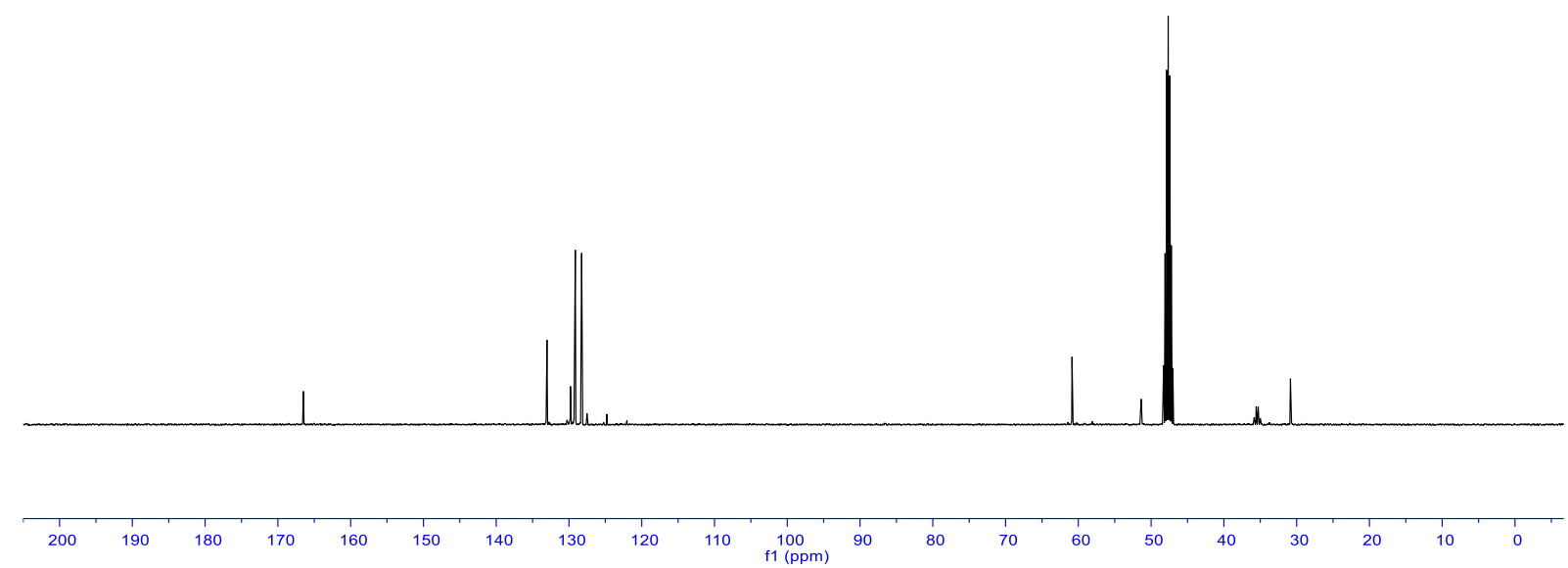


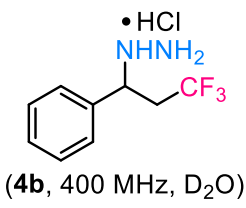

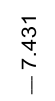

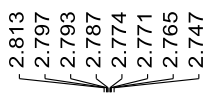
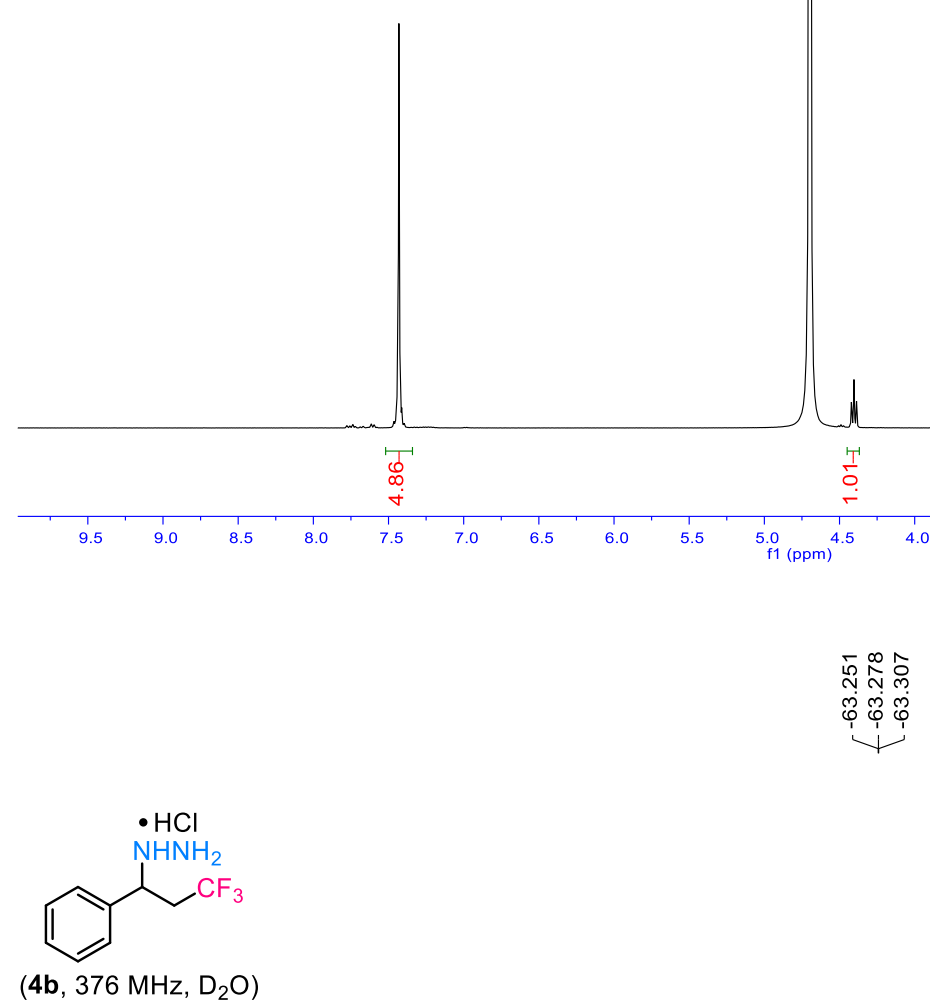

(4b, $376 \mathrm{MHz}, \mathrm{D}_{2} \mathrm{O}$ )

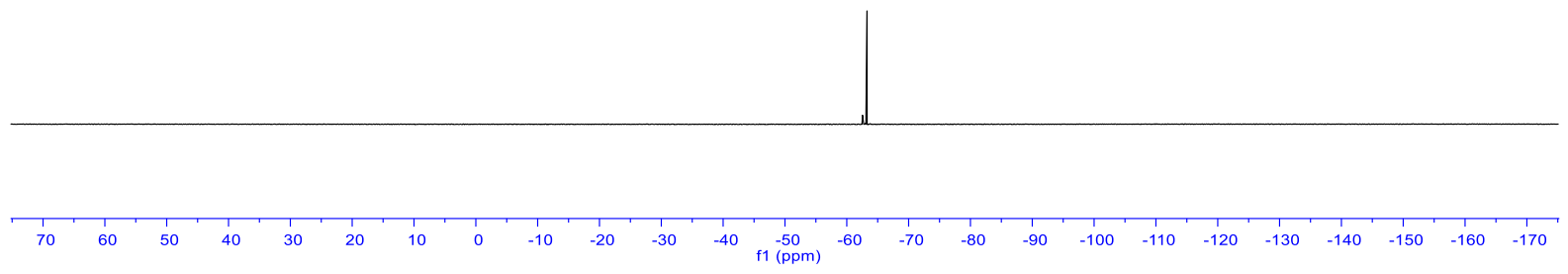

S29 

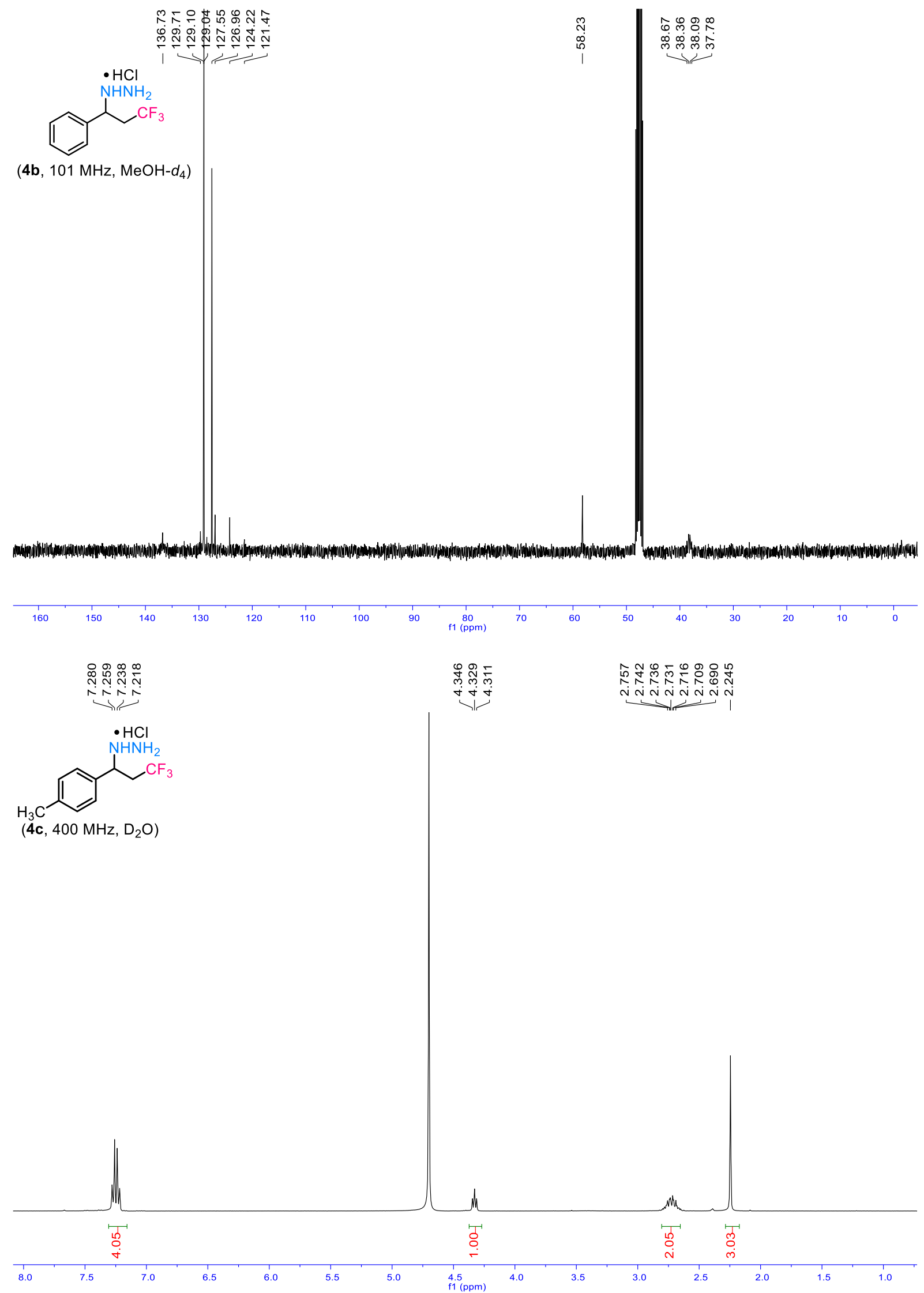


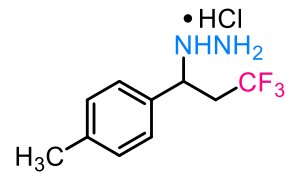

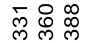

फ़्फ

(4c, $376 \mathrm{MHz}, \mathrm{D}_{2} \mathrm{O}$ )

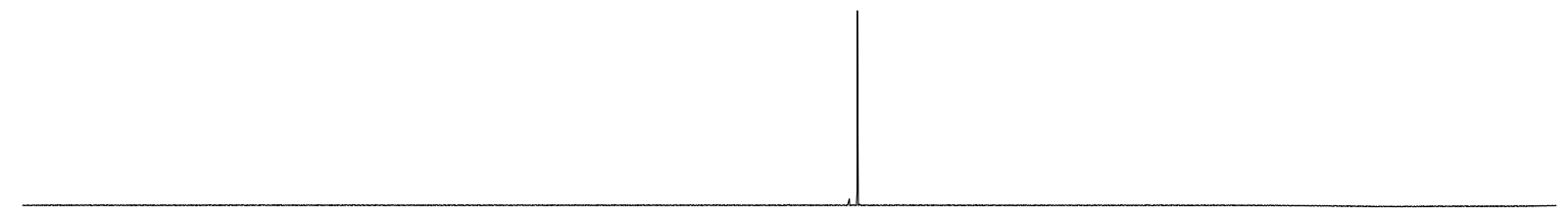

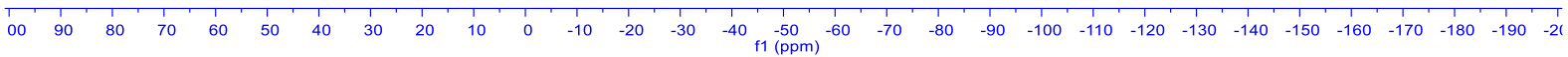

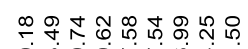

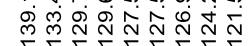

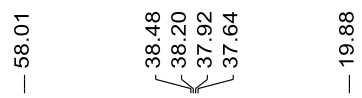

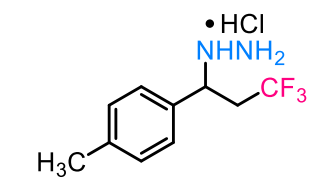

(4c, $101 \mathrm{MHz}, \mathrm{MeOH}-d_{4}$ )
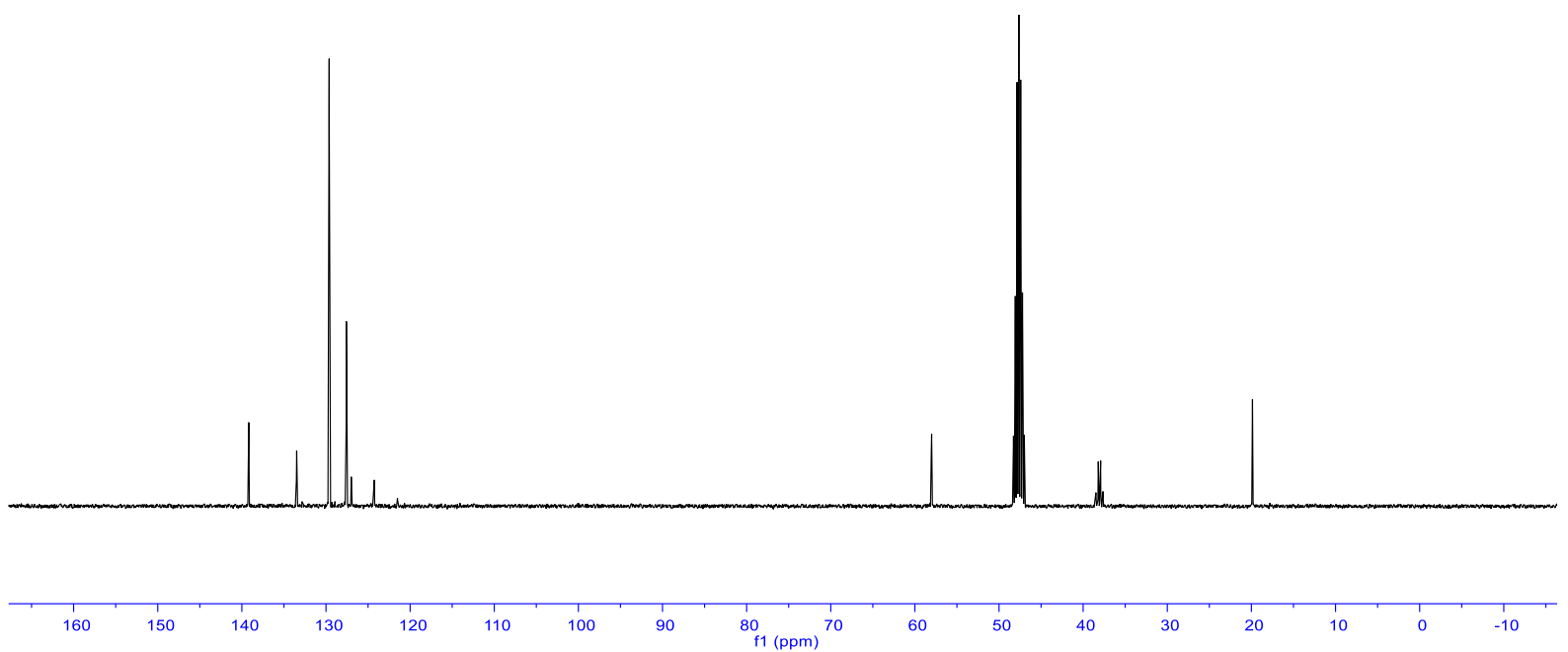


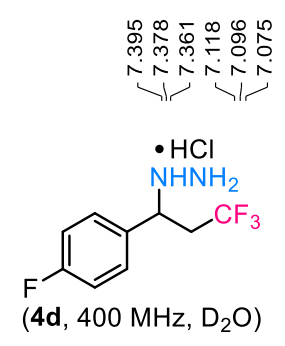

綒员品

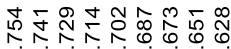

ง กงกง

(4d, $400 \mathrm{MHz}, \mathrm{D}_{2} \mathrm{O}$ )
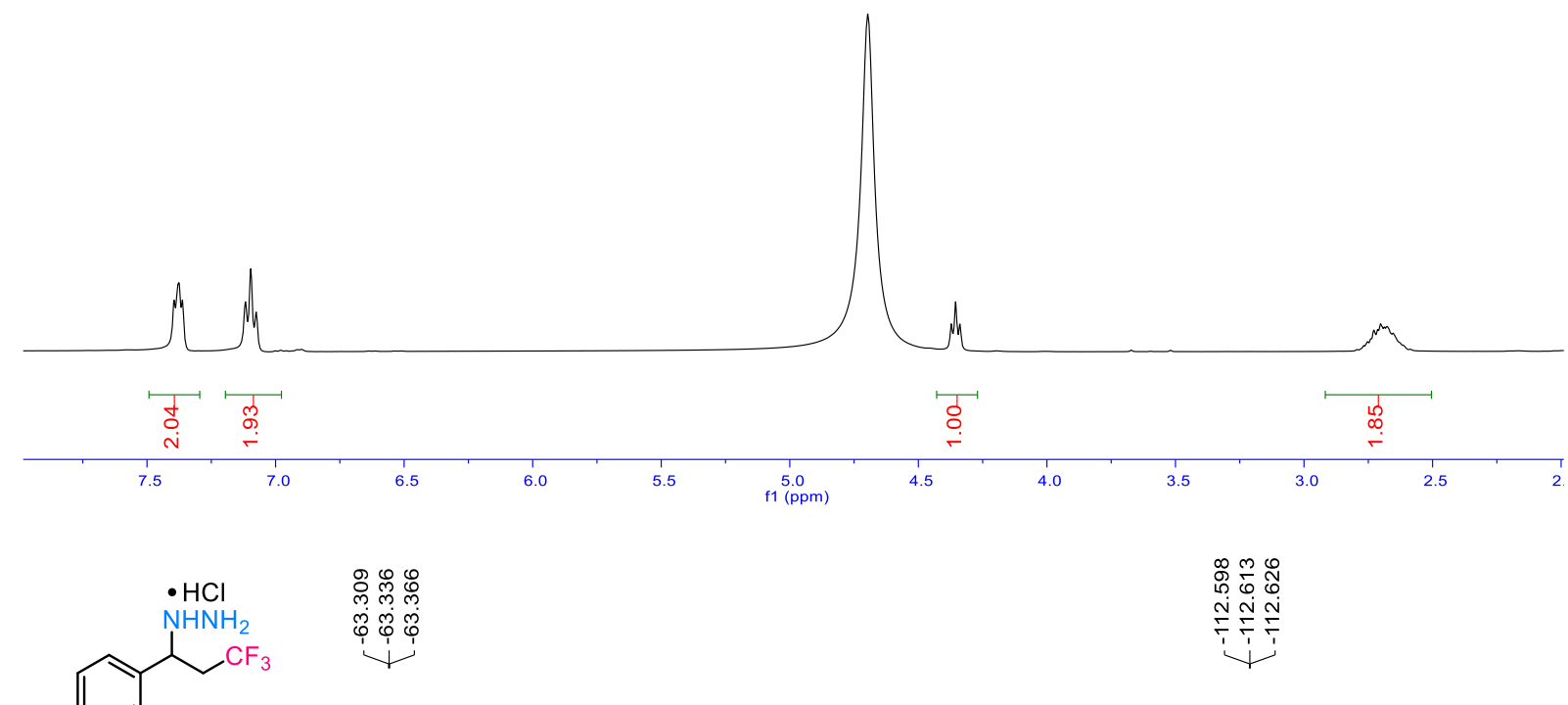

(4d, $376 \mathrm{MHz}, \mathrm{D}_{2} \mathrm{O}$ )

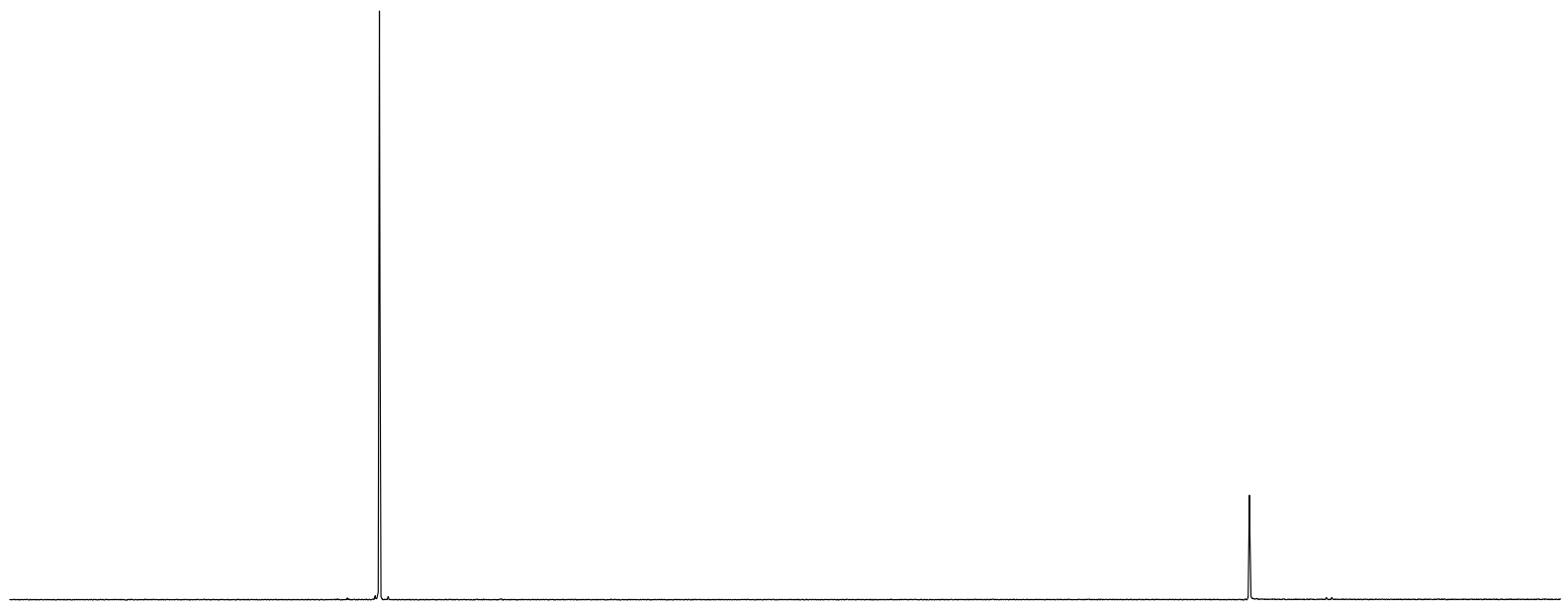

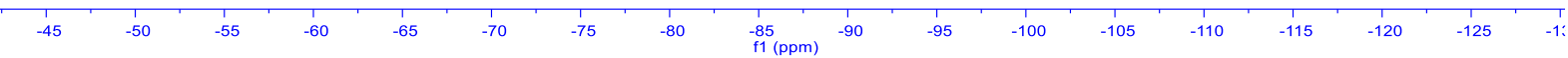



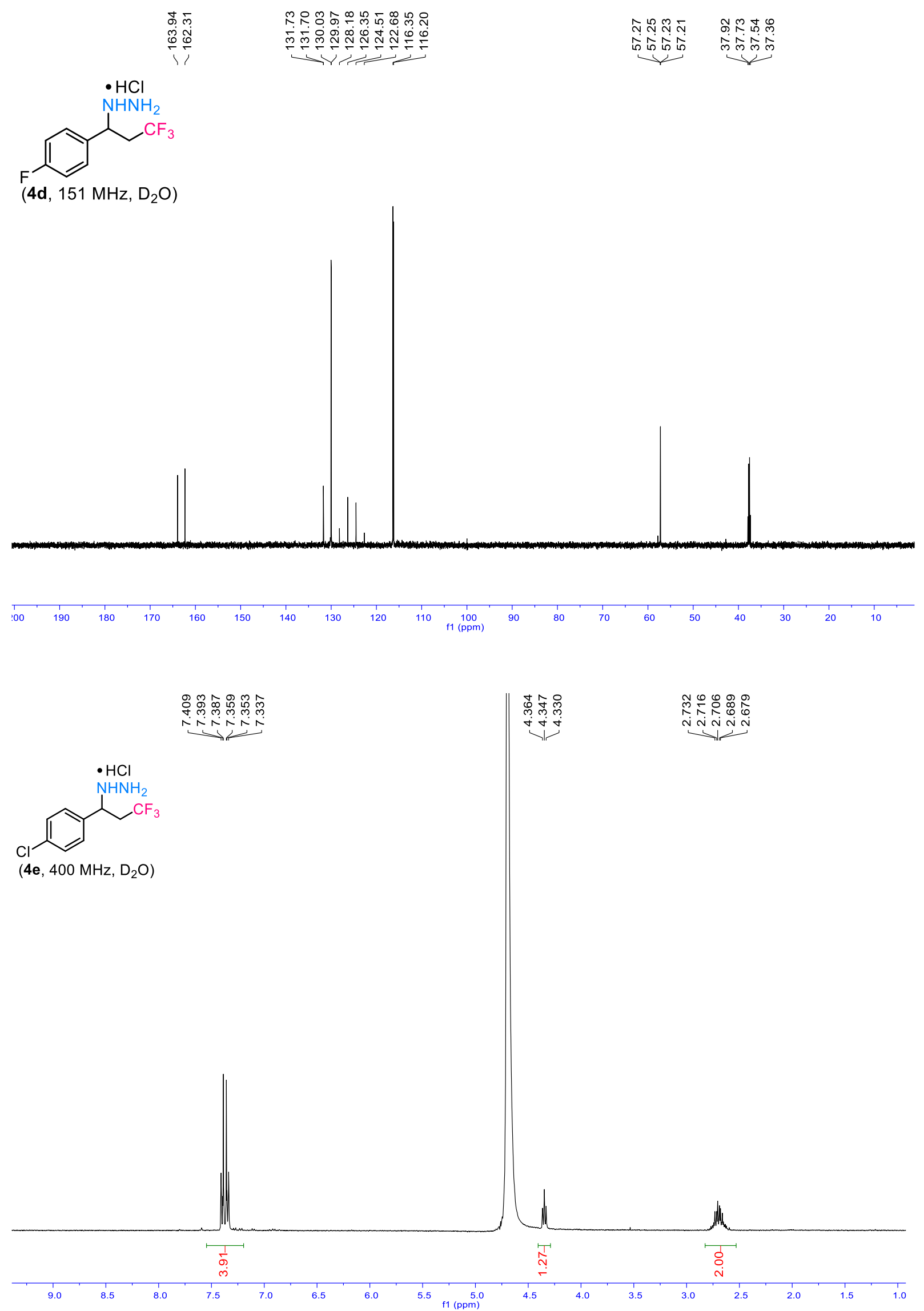


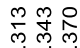

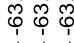

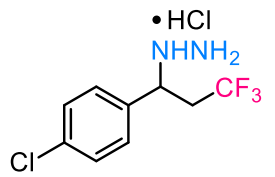

$\left(4 \mathbf{e}, 376 \mathrm{MHz}, \mathrm{D}_{2} \mathrm{O}\right)$

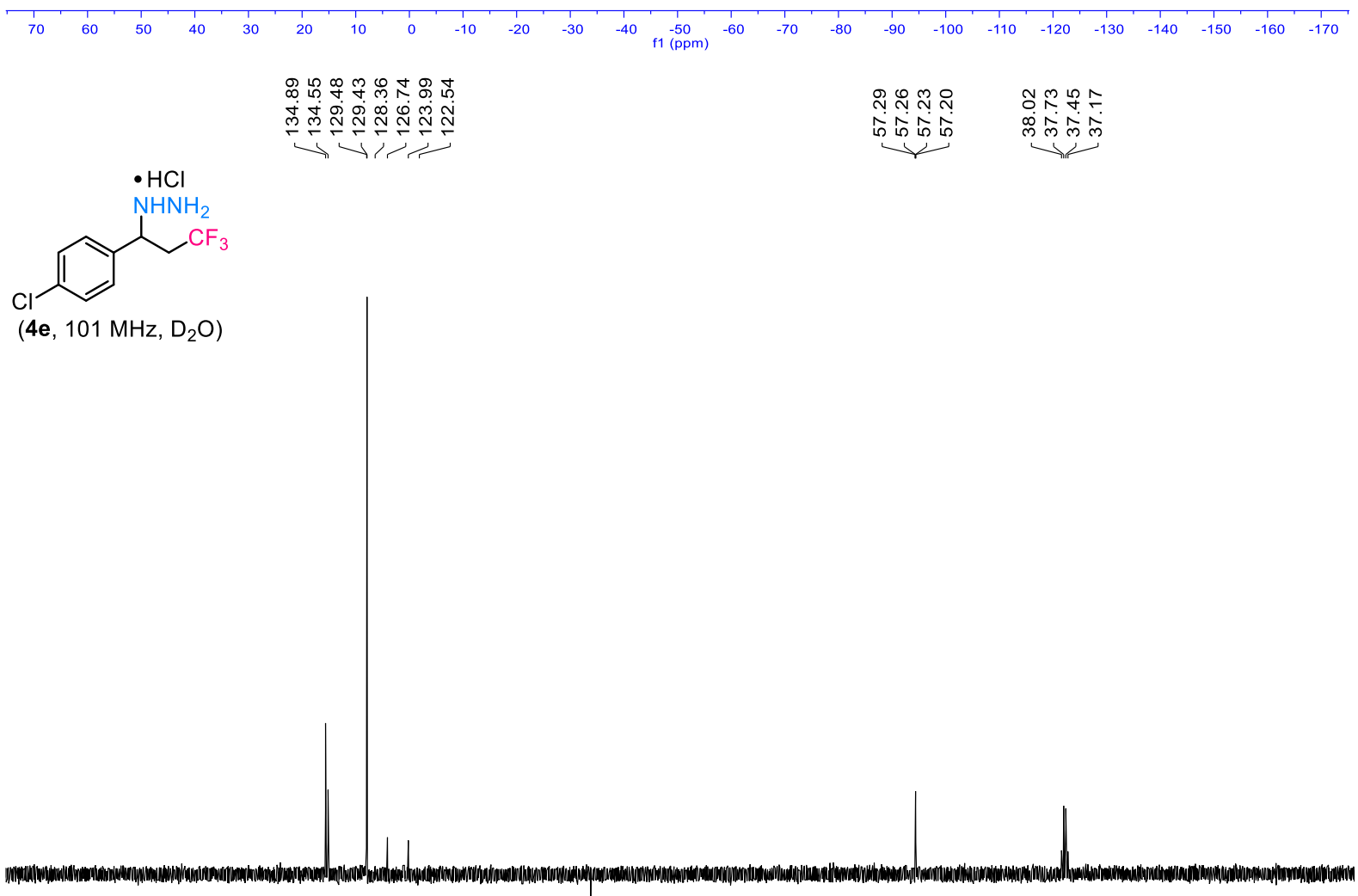

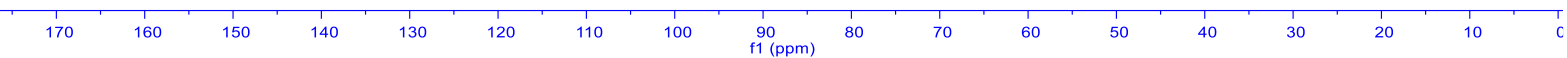




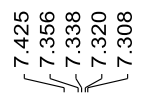

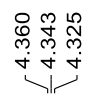

$\underbrace{2}$

$\left(4 \mathrm{Cl}, 400 \mathrm{MHz}, \mathrm{D}_{2} \mathrm{O}\right.$
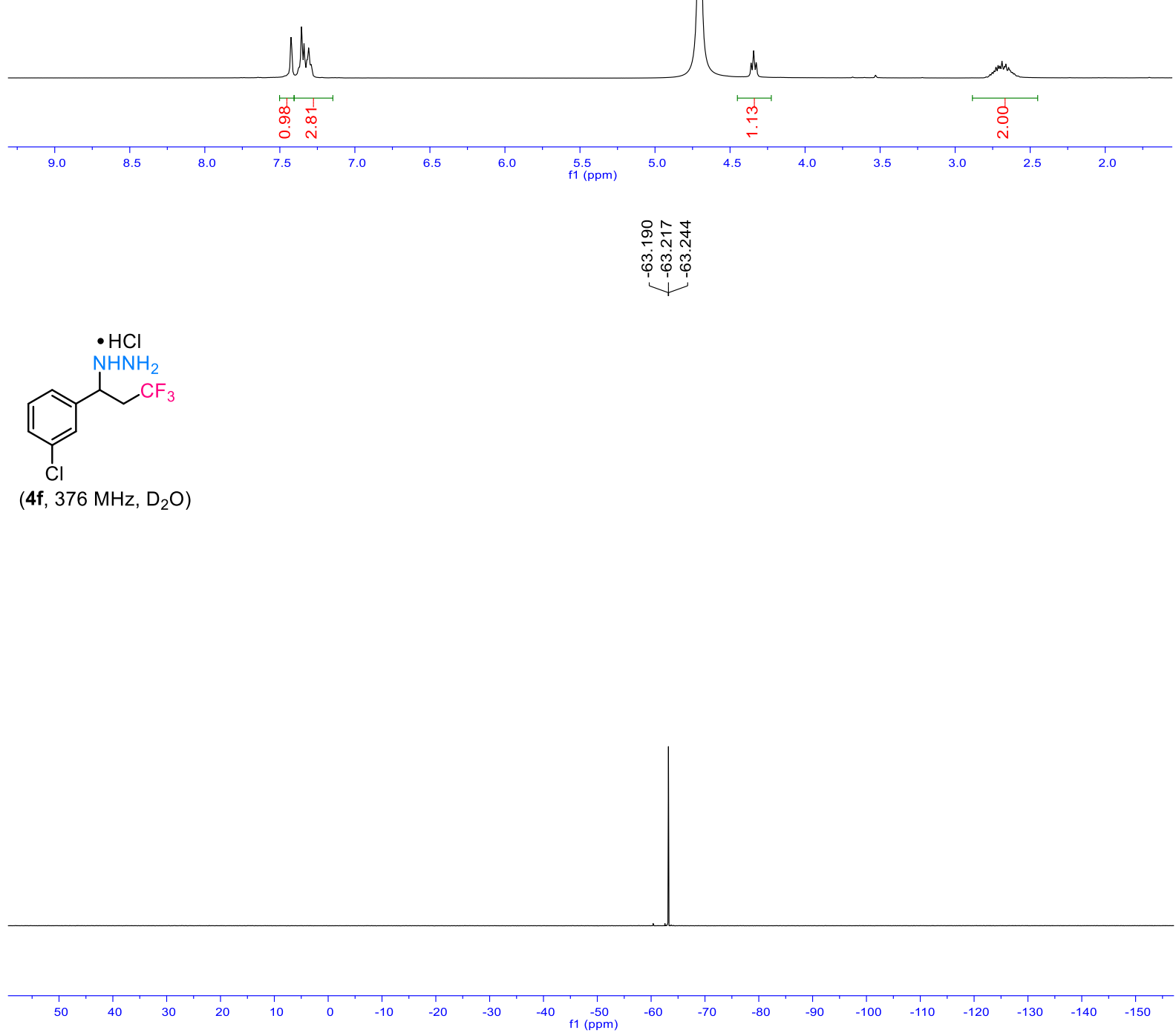

S35 


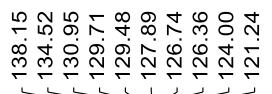

$\underbrace{0}_{0}$

$\left(4 \mathrm{f}, 101 \mathrm{MHz}, \mathrm{D}_{2} \mathrm{O}\right)$
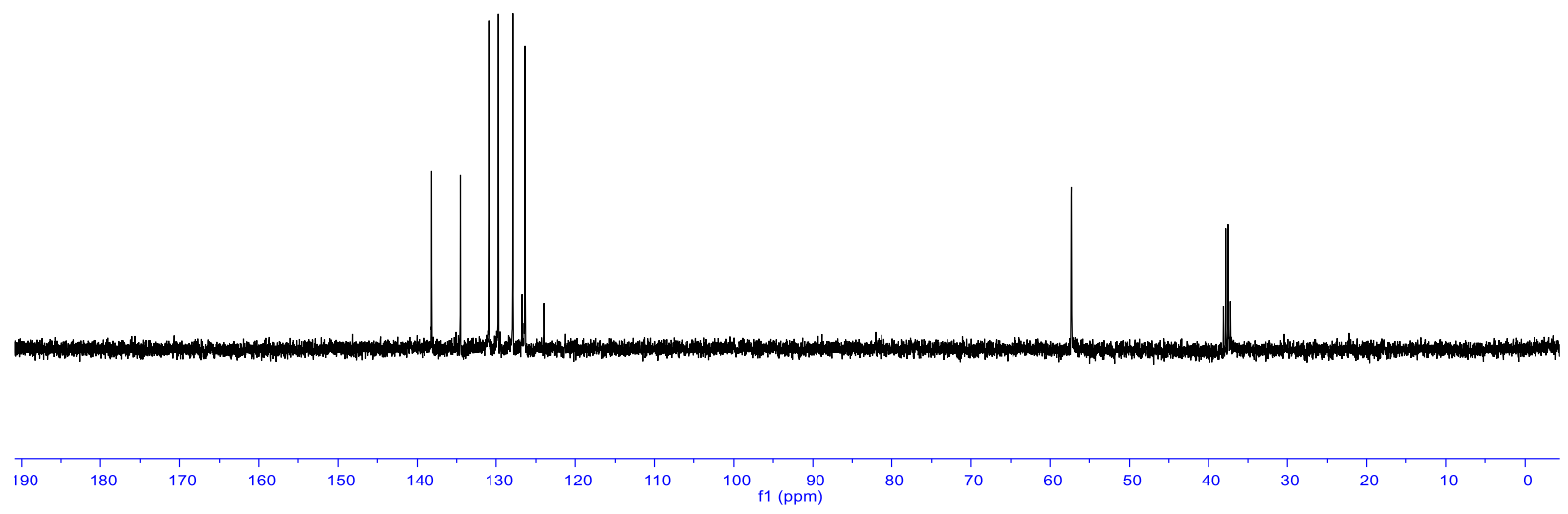

勿

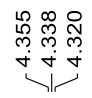

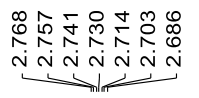

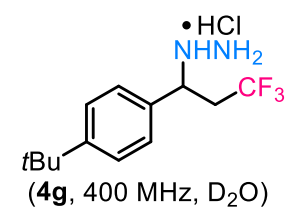

(4g, $400 \mathrm{MHz}, \mathrm{D}_{2} \mathrm{O}$ )

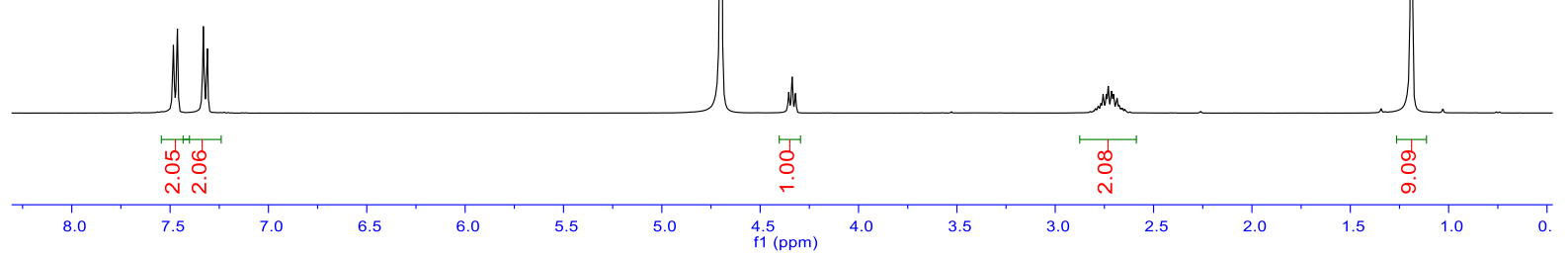

S36 


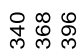

గ్ధ

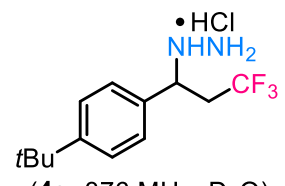

$\left(\mathbf{4 g}, 376 \mathrm{MHz}, \mathrm{D}_{2} \mathrm{O}\right)$

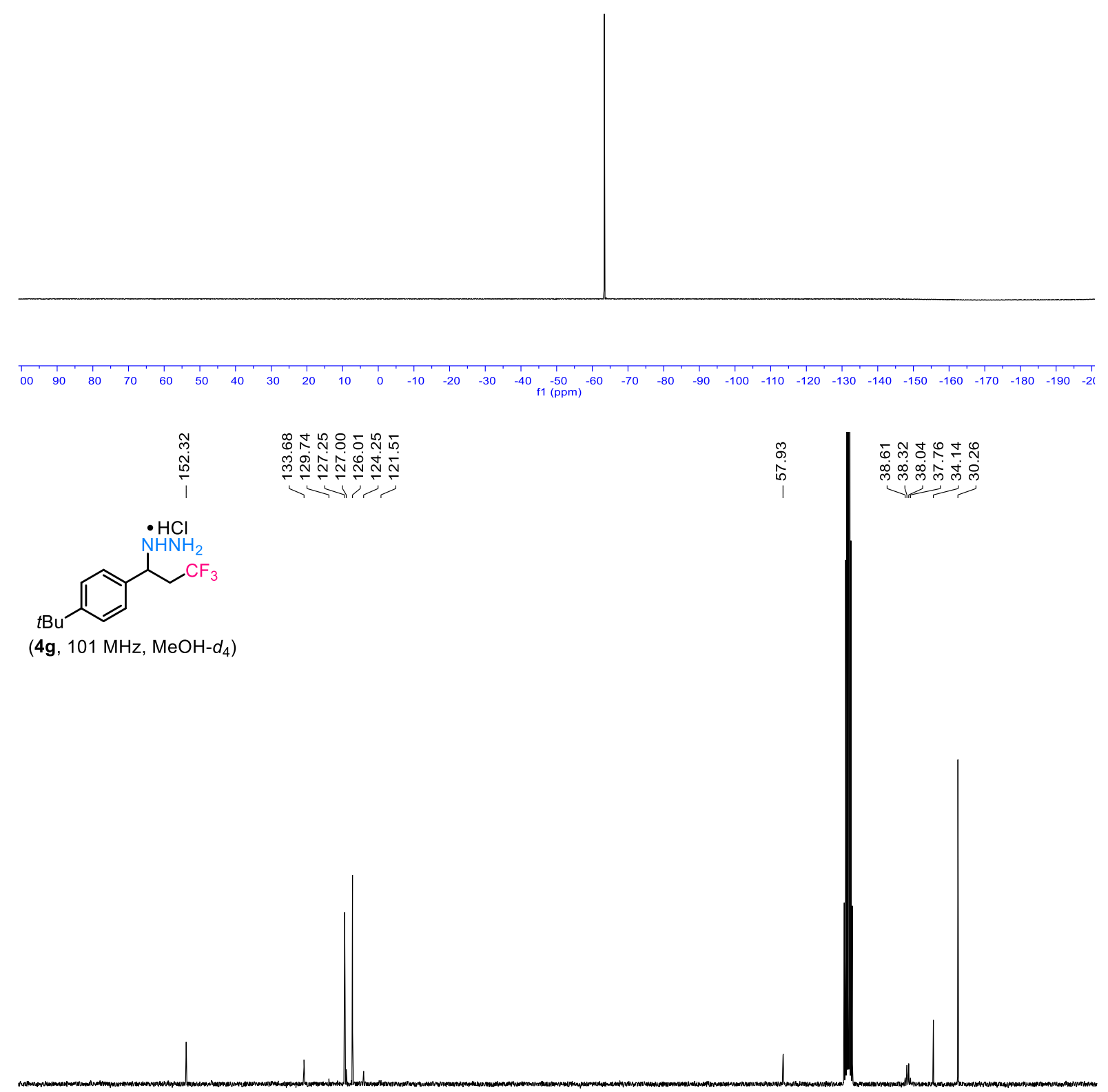

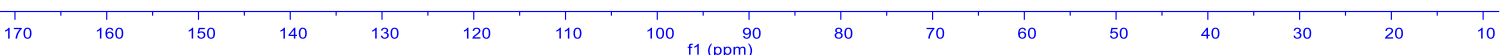




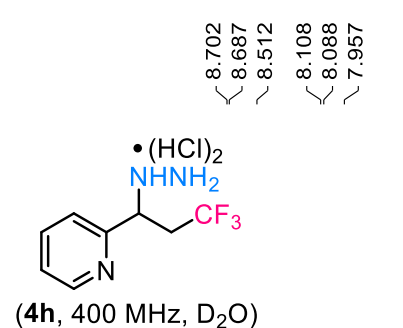

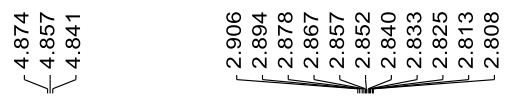

(4h, $400 \mathrm{MHz}, \mathrm{D}_{2} \mathrm{O}$ )
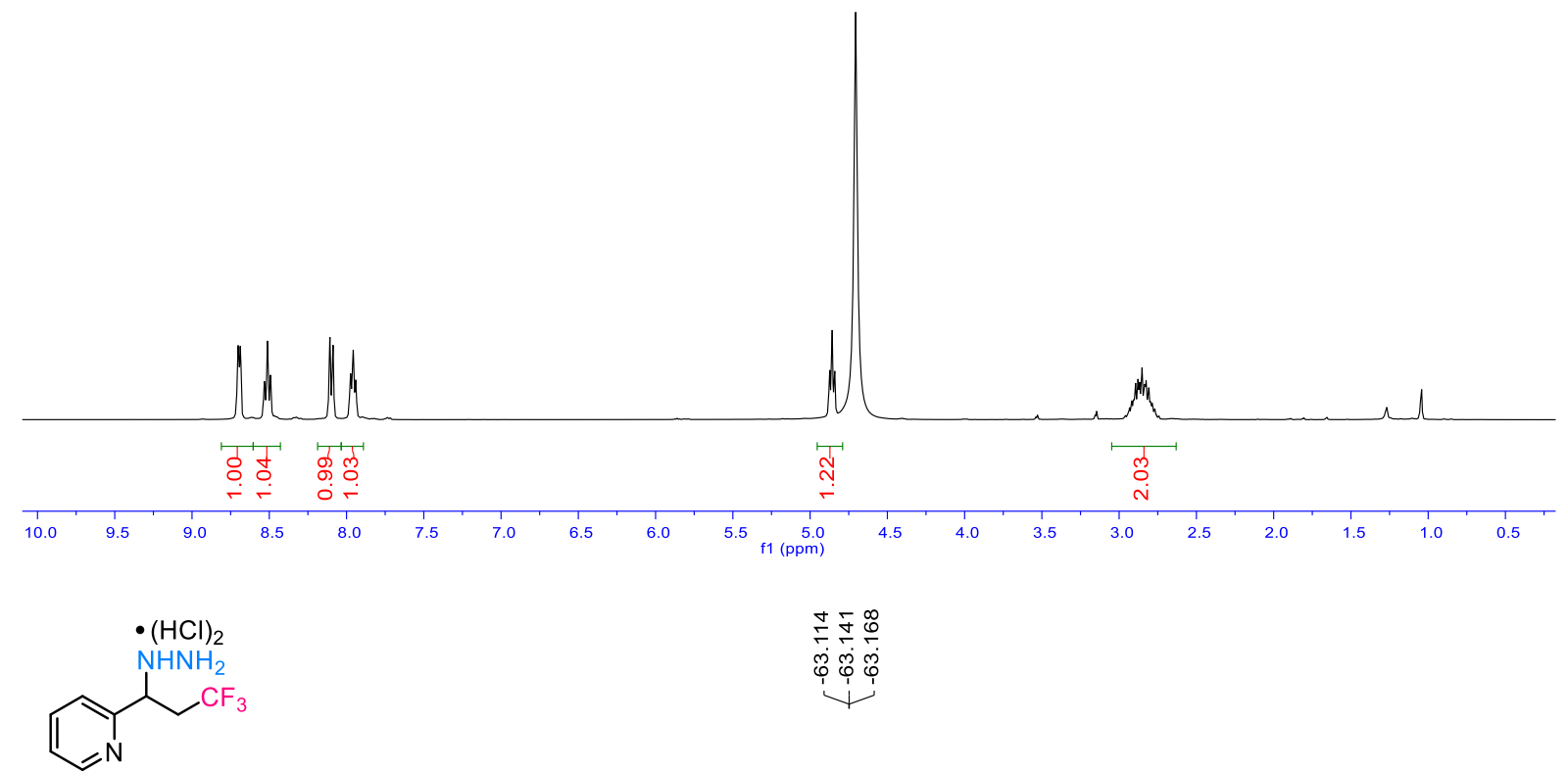

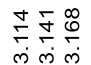

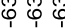

(4h, $376 \mathrm{MHz}, \mathrm{D}_{2} \mathrm{O}$ )

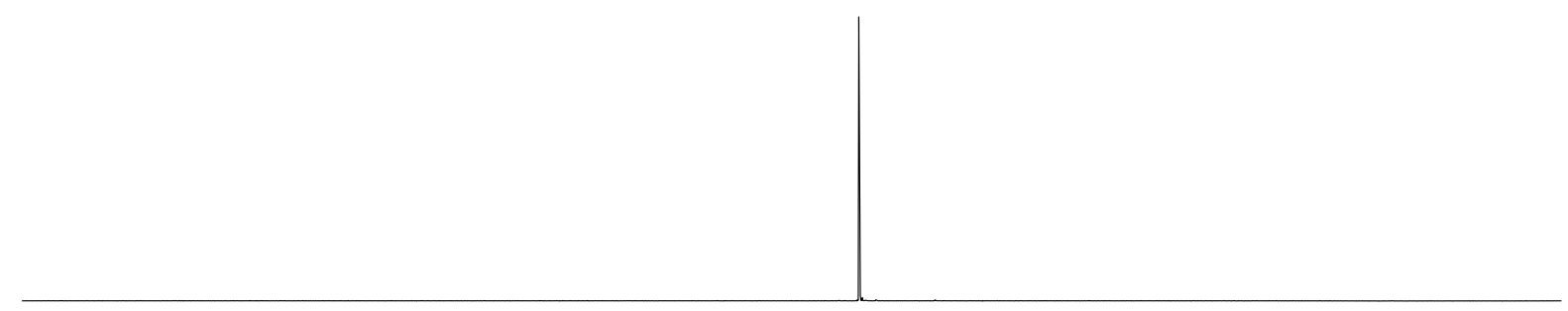

\begin{tabular}{llllllllllllllllllllllllllllllllllllll}
\hline 0 & 90 & 80 & 70 & 60 & 50 & 40 & 30 & 20 & 10 & 0 & -10 & -20 & -30 & -40 & -50 & -60 & -70 & -80 & -90 & -100 & -110 & -120 & -130 & -140 & -150 & -160 & -170 & -180 & -190 & -21
\end{tabular} 

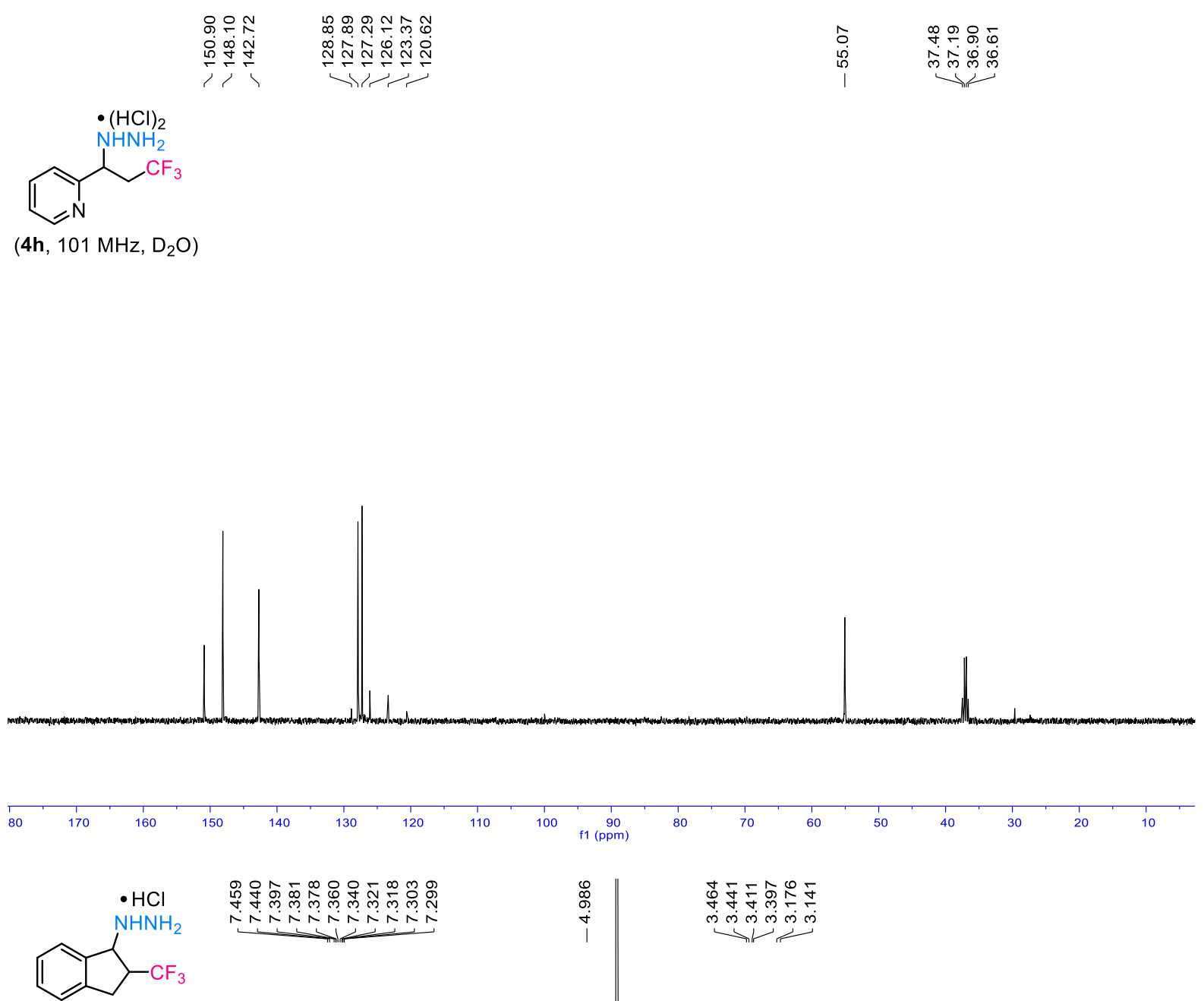

(4i, $400 \mathrm{MHz}, \mathrm{D}_{2} \mathrm{O}$ )
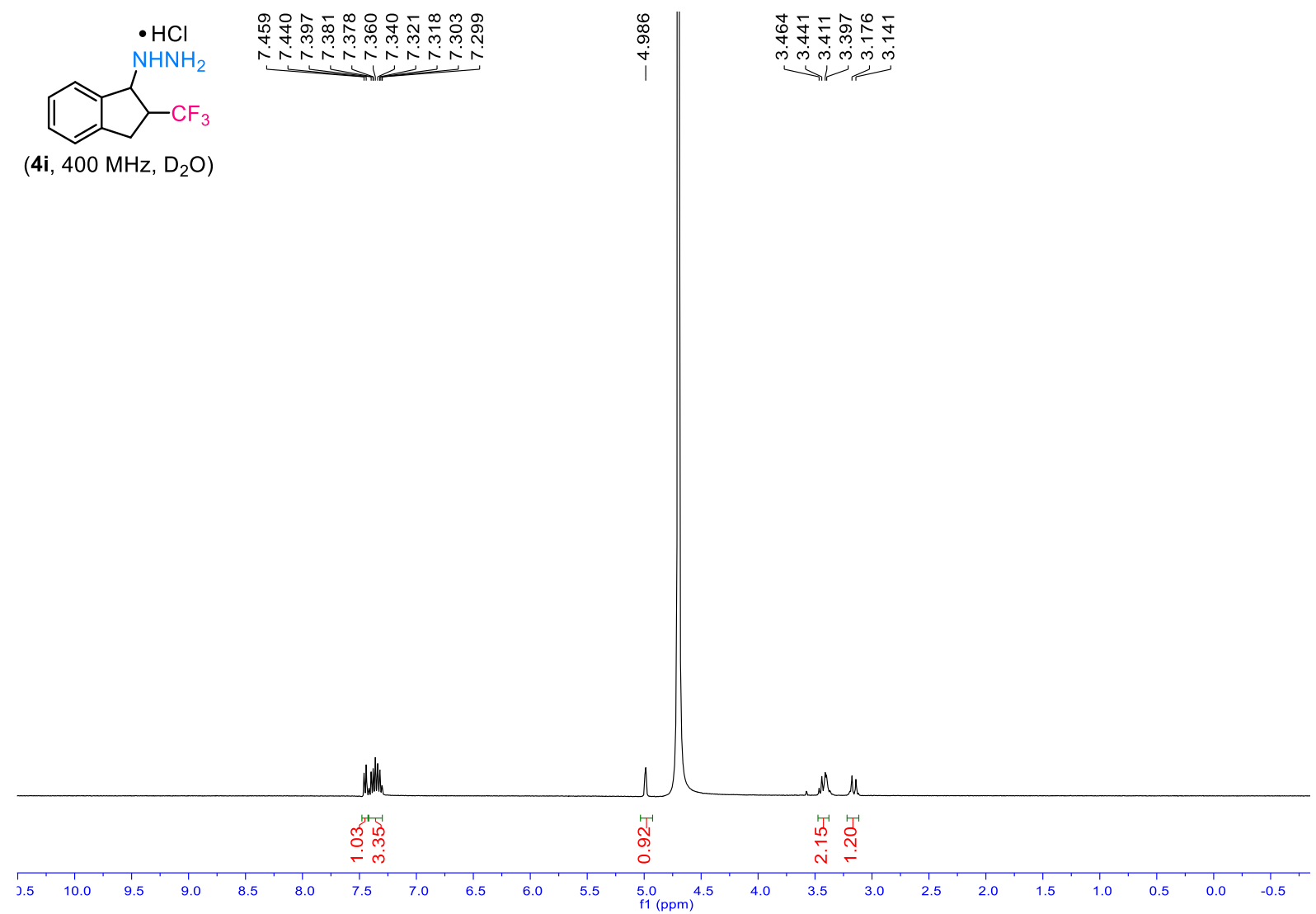


\section{尔离}

ì

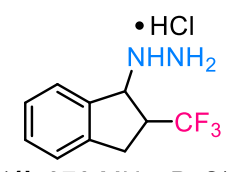

$\left(4 \mathrm{i}, 376 \mathrm{MHz}, \mathrm{D}_{2} \mathrm{O}\right)$
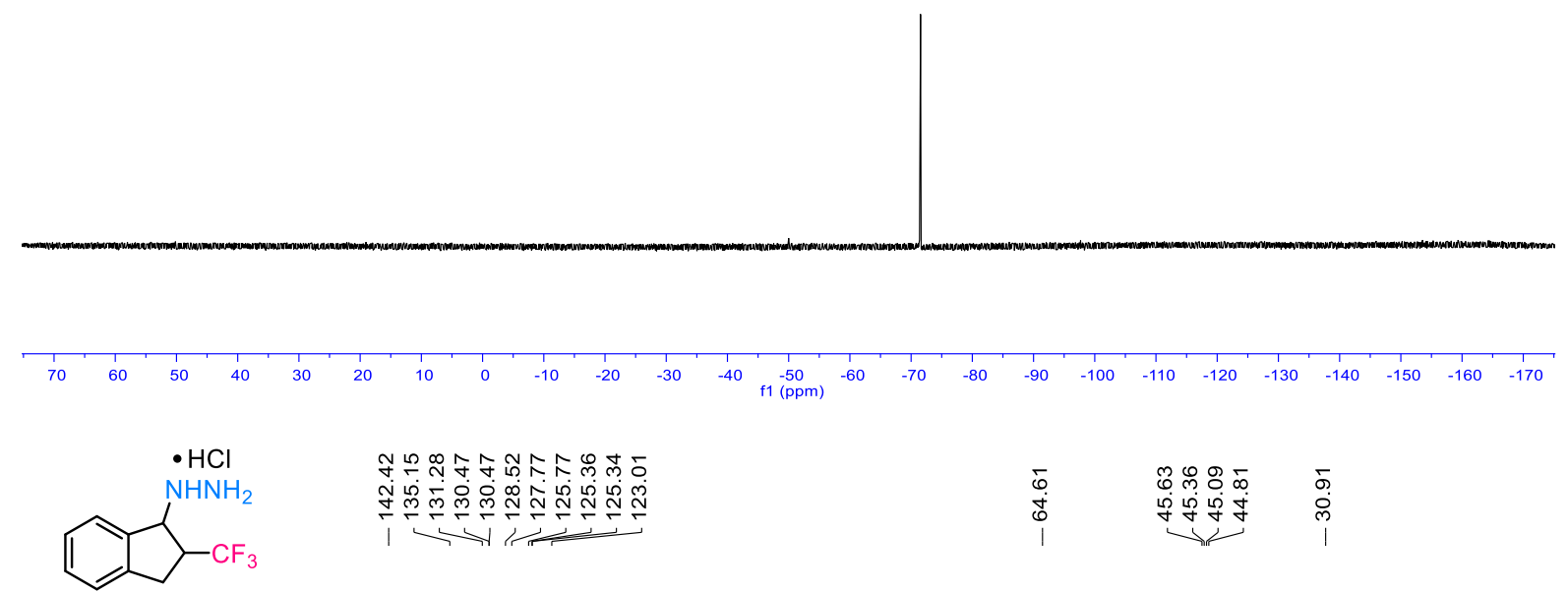

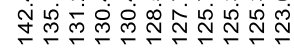

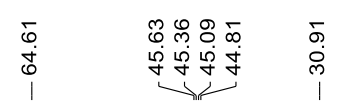

(4i, $101 \mathrm{MHz}, \mathrm{D}_{2} \mathrm{O}$ )

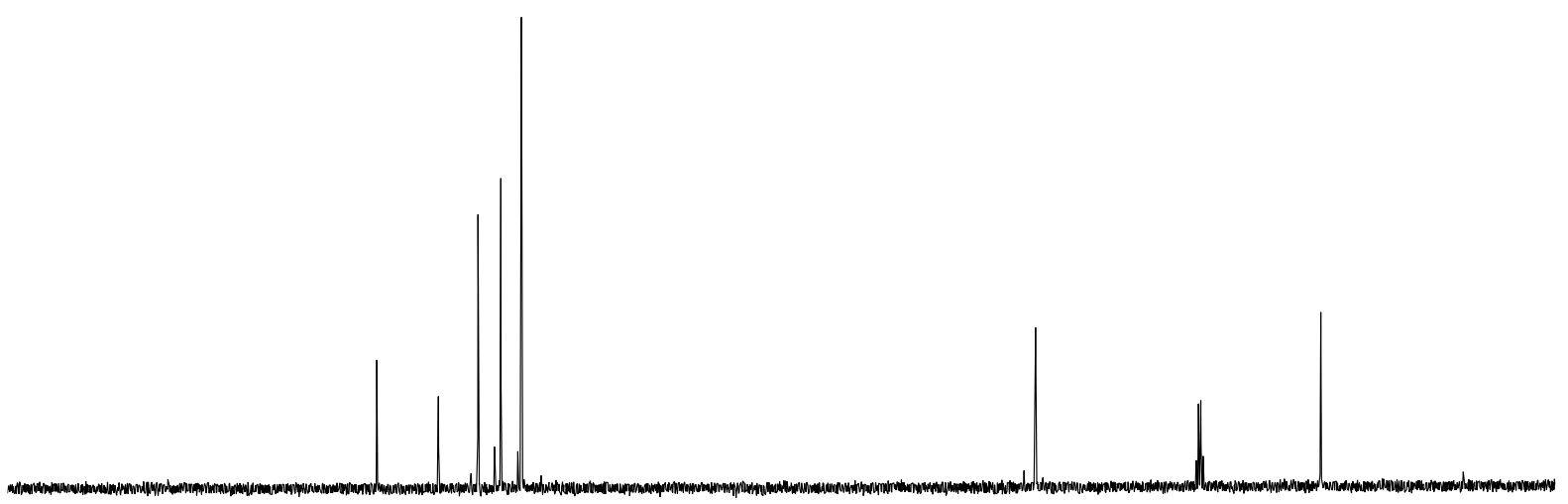

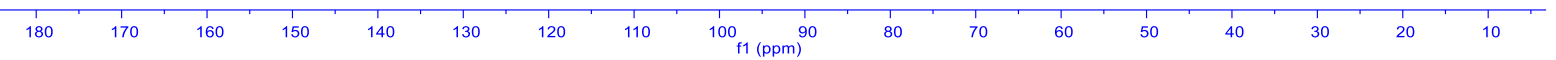




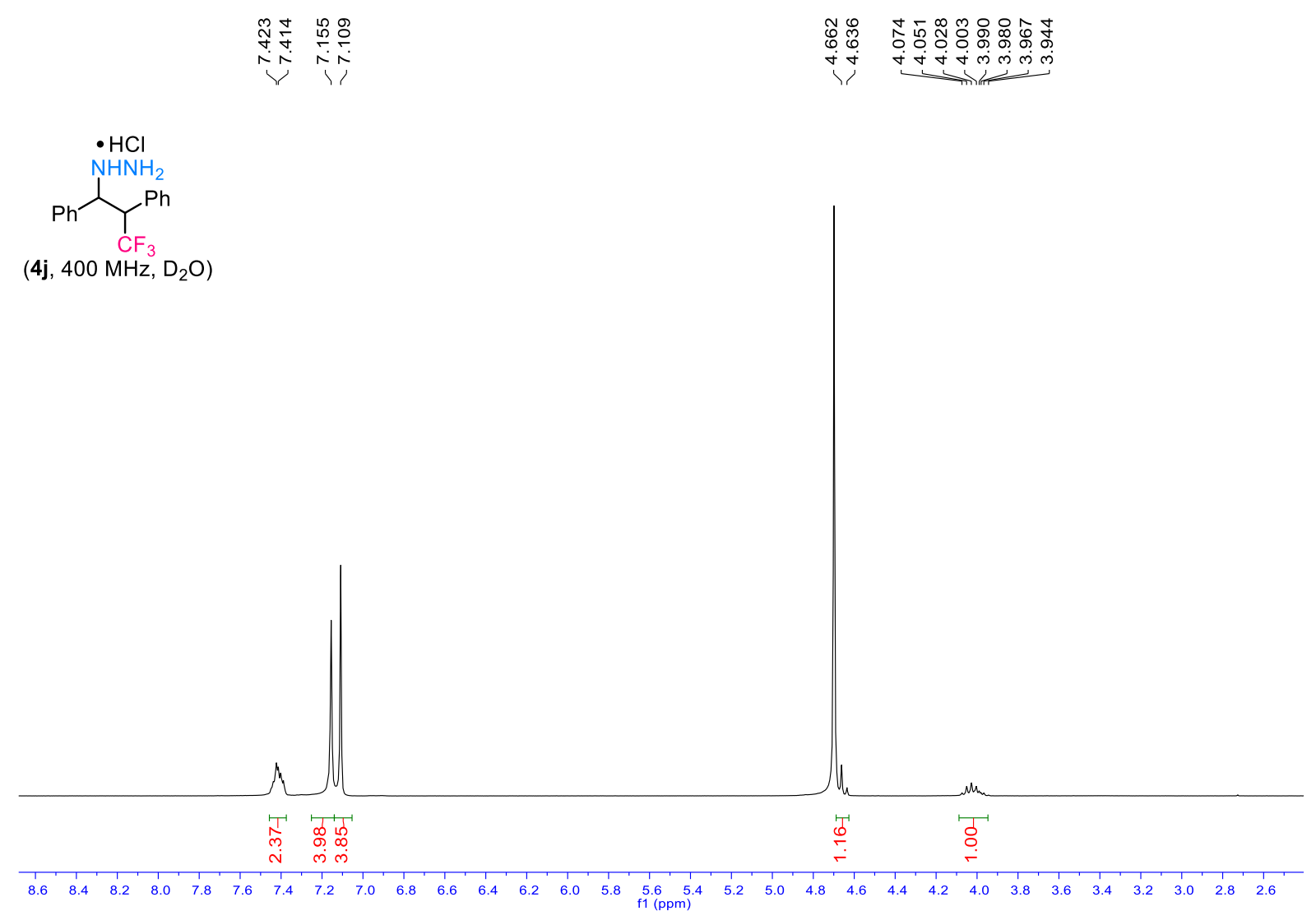

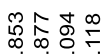

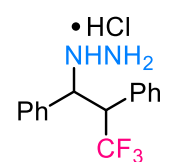

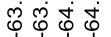

柁

(4j, $376 \mathrm{MHz}, \mathrm{D}_{2} \mathrm{O}$ )

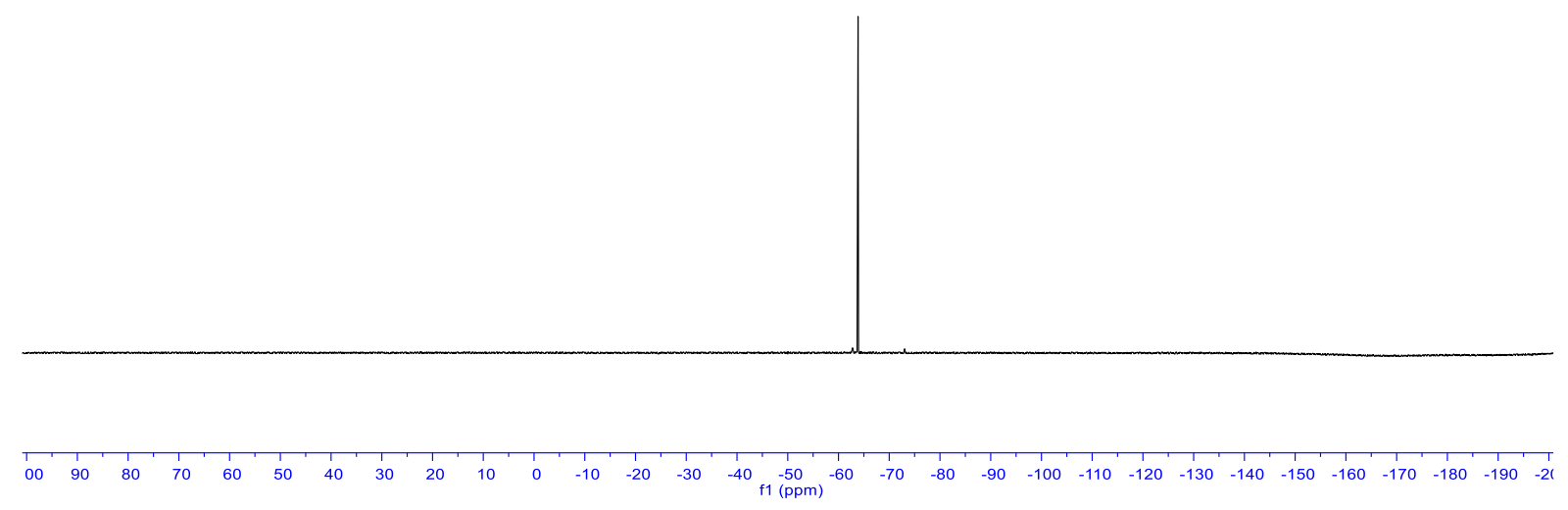




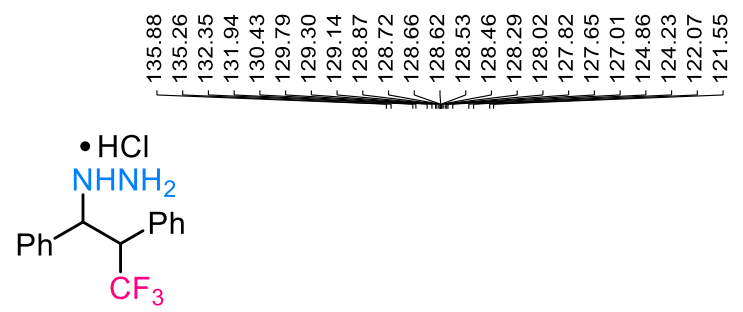

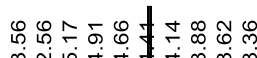

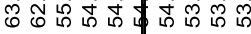

(4j, $\left.101 \mathrm{MHz}, \mathrm{MeOH}-d_{4}\right)$
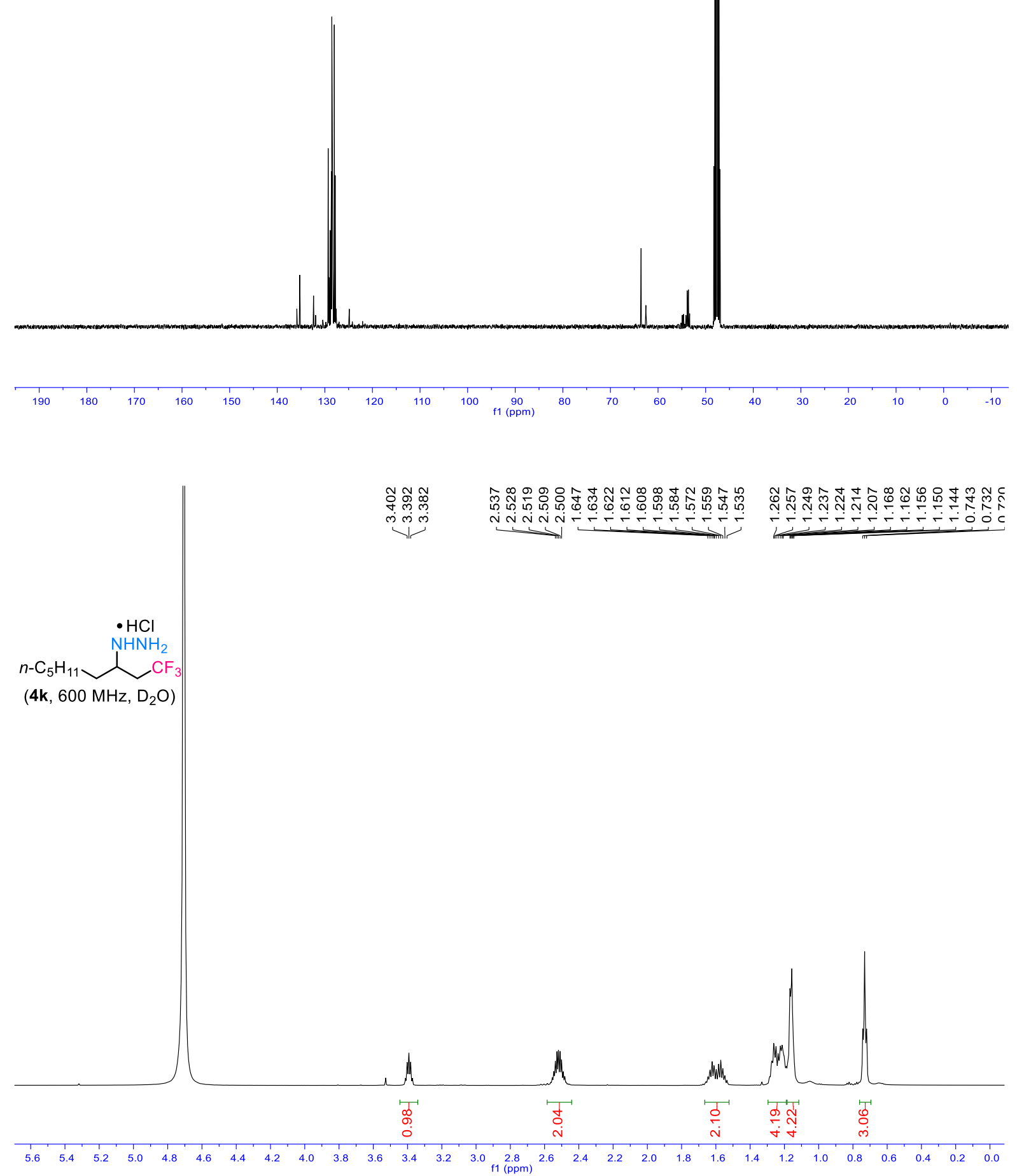


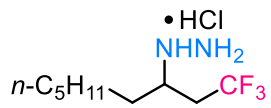

(4k, $565 \mathrm{MHz}, \mathrm{D}_{2} \mathrm{O}$ )
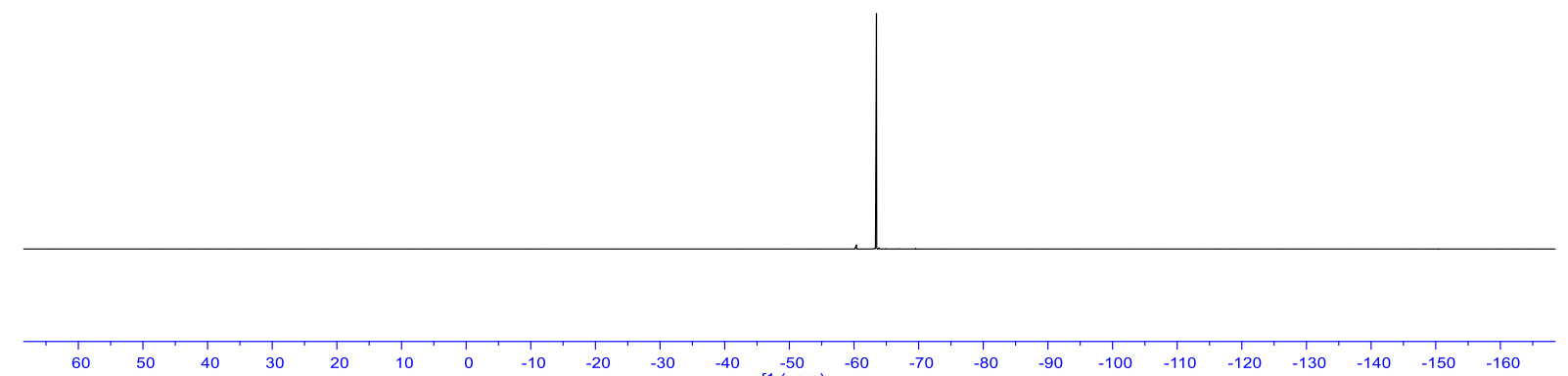

องกำกำ

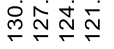

요요요

过它乱我

1111

है की एक

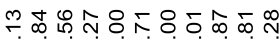

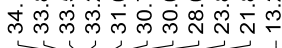

- $\mathrm{HCl}$

$\mathrm{NHNH}_{2}$

$n-\mathrm{C}_{5} \mathrm{H}_{11} \longrightarrow \mathrm{CF}_{3}$

(4k, $101 \mathrm{MHz}, \mathrm{D}_{2} \mathrm{O}$ )

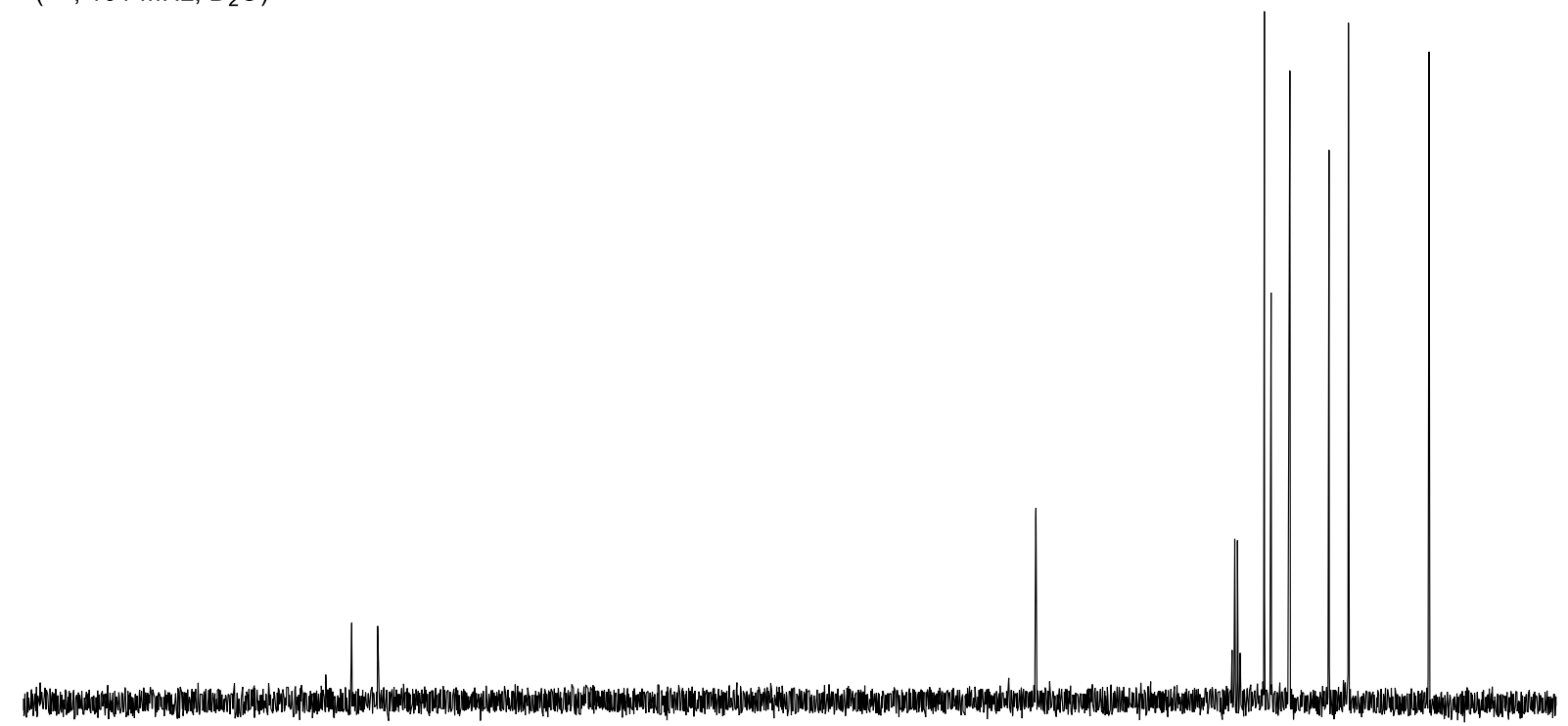

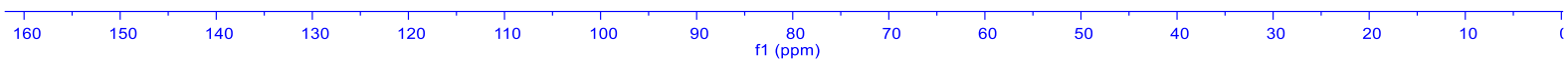




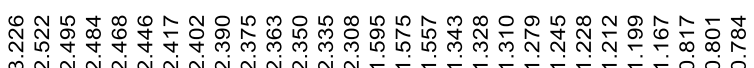

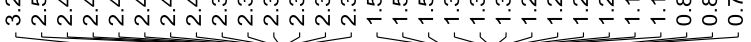

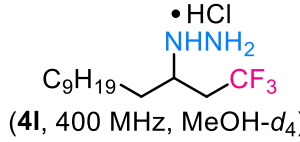

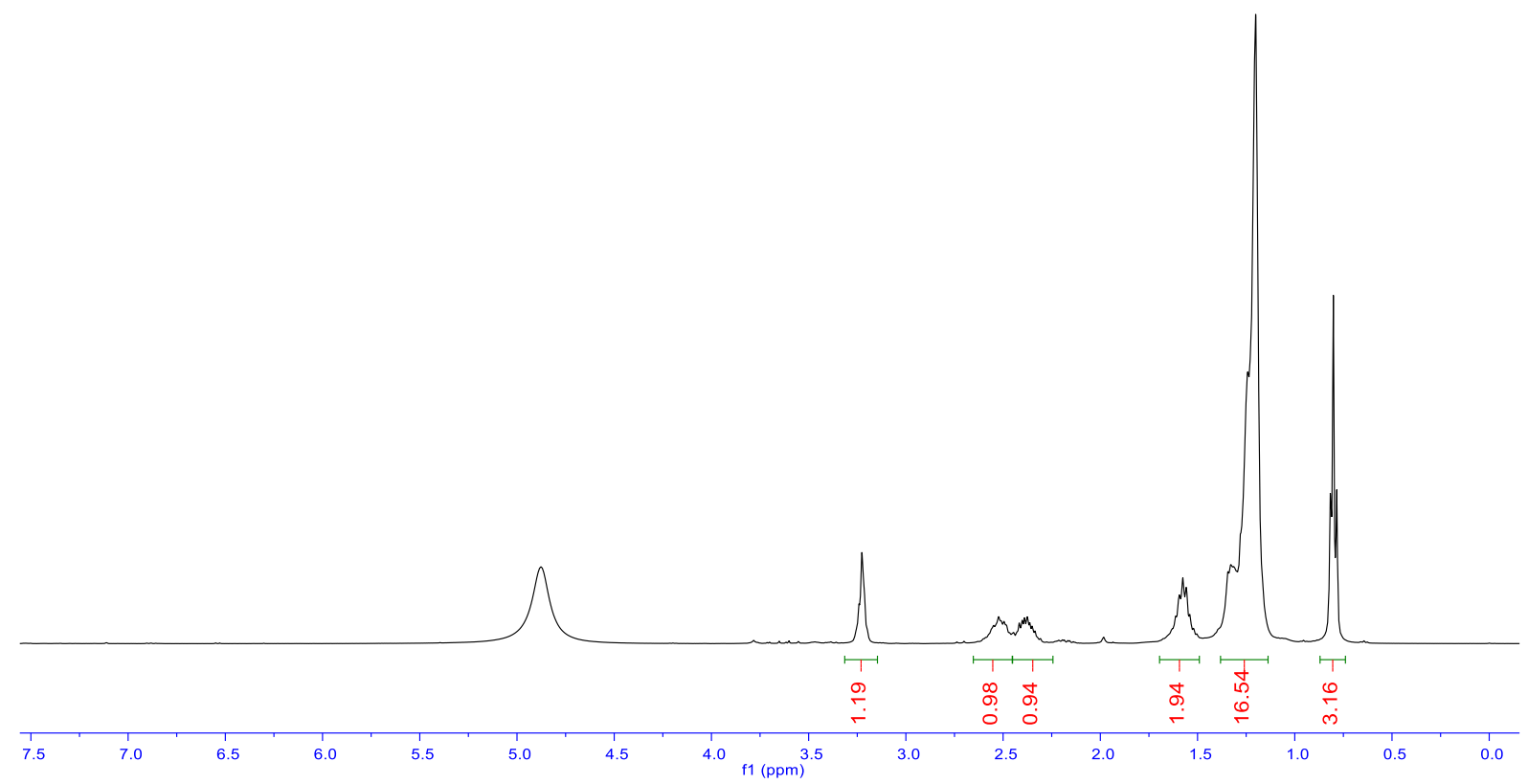

รูำ

它安导

$\mathrm{C}_{9} \mathrm{H}_{19} \stackrel{\bullet \mathrm{HCl}}{\mathrm{NHNH}_{2}} \mathrm{CF}_{3}$

(4I, $\left.376 \mathrm{MHz}, \mathrm{MeOH}-d_{4}\right)$

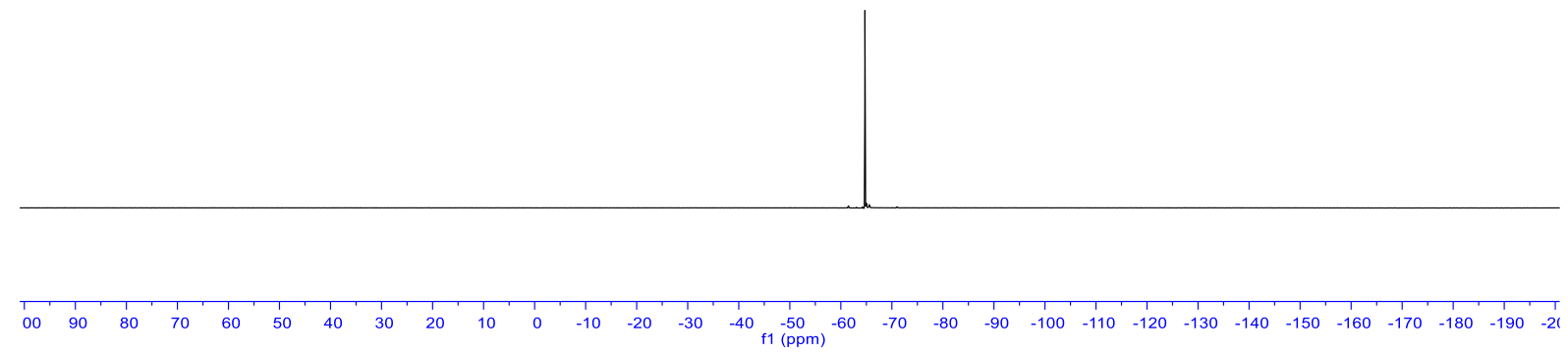




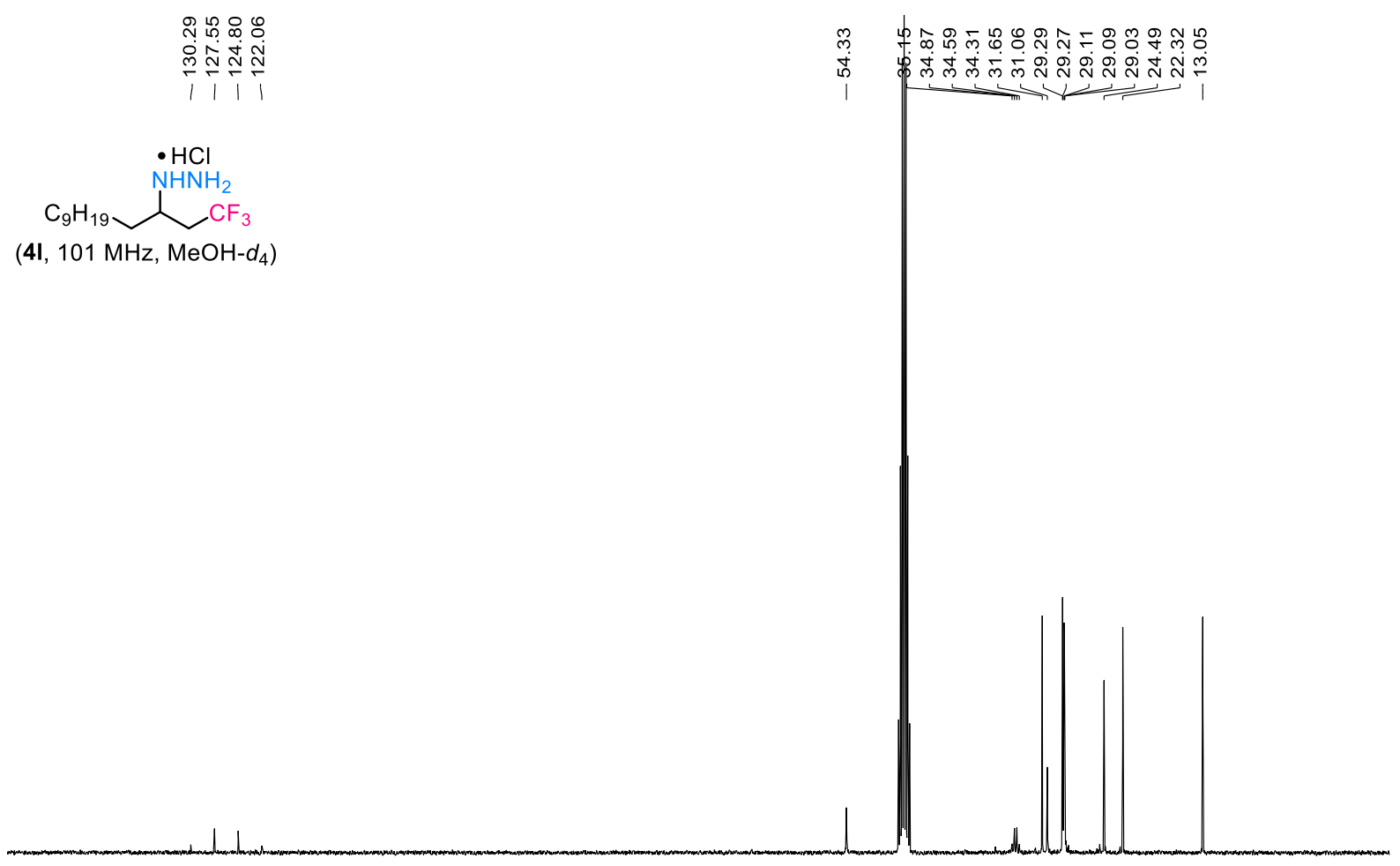

กำ

ํำ

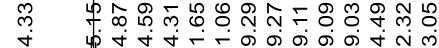

பำ

$\mathrm{C}_{9} \mathrm{H}_{19} \longrightarrow \mathrm{CF}_{3}$

(4I, $101 \mathrm{MHz}, \mathrm{MeOH}-d_{4}$ )

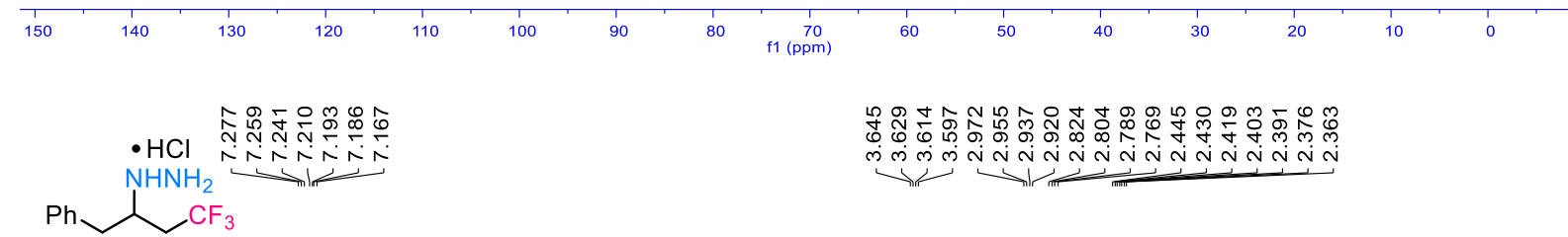

(4m, $400 \mathrm{MHz}, \mathrm{D}_{2} \mathrm{O}$ )

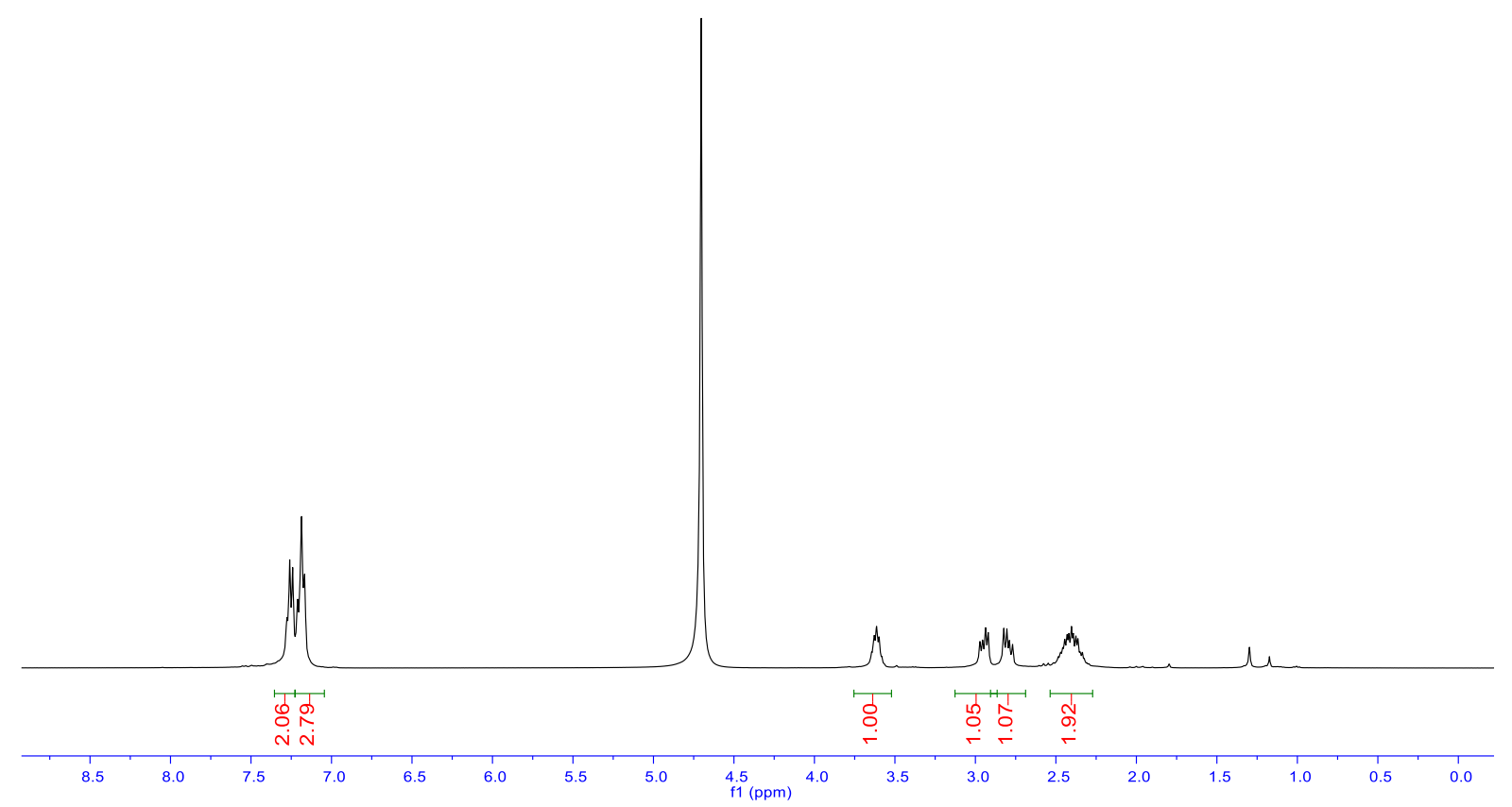




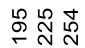

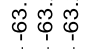

- $\mathrm{HCl}$

$\overbrace{(4)}^{\mathrm{NHNH}_{2}}$

$\left(4 \mathrm{~m}, 376 \mathrm{MHz}, \mathrm{D}_{2} \mathrm{O}\right)$

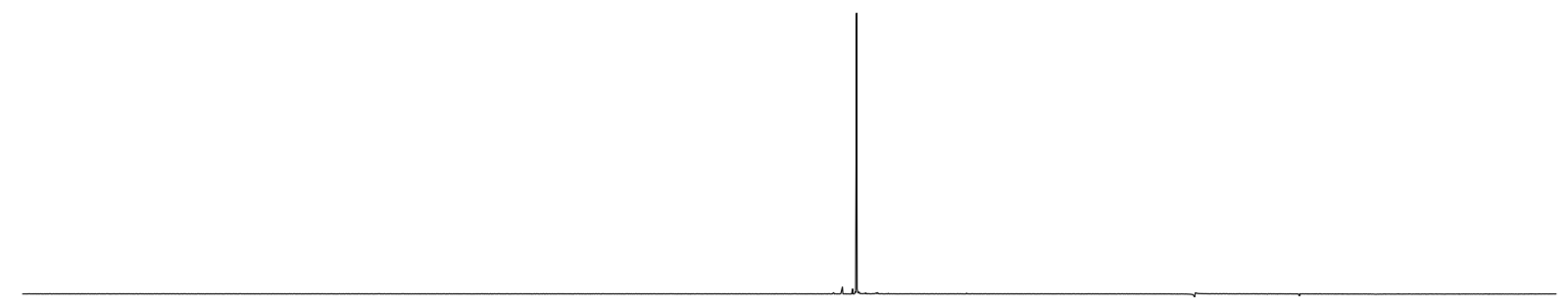

$\begin{array}{lllllllllllllllllllllllllllllllllllll}00 & 90 & 80 & 70 & 60 & 50 & 40 & 30 & 20 & 10 & 0 & -10 & -20 & -30 & -40 & -50 & -60 & -70 & -80 & -90 & -100 & -110 & -120 & -130 & -140 & -150 & -160 & -170 & -180 & -190 & -21\end{array}$

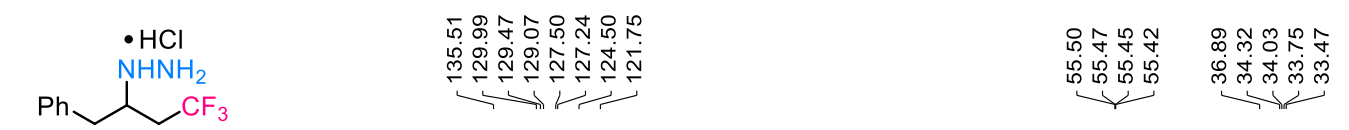

(4m, $101 \mathrm{MHz}, \mathrm{D}_{2} \mathrm{O}$ )

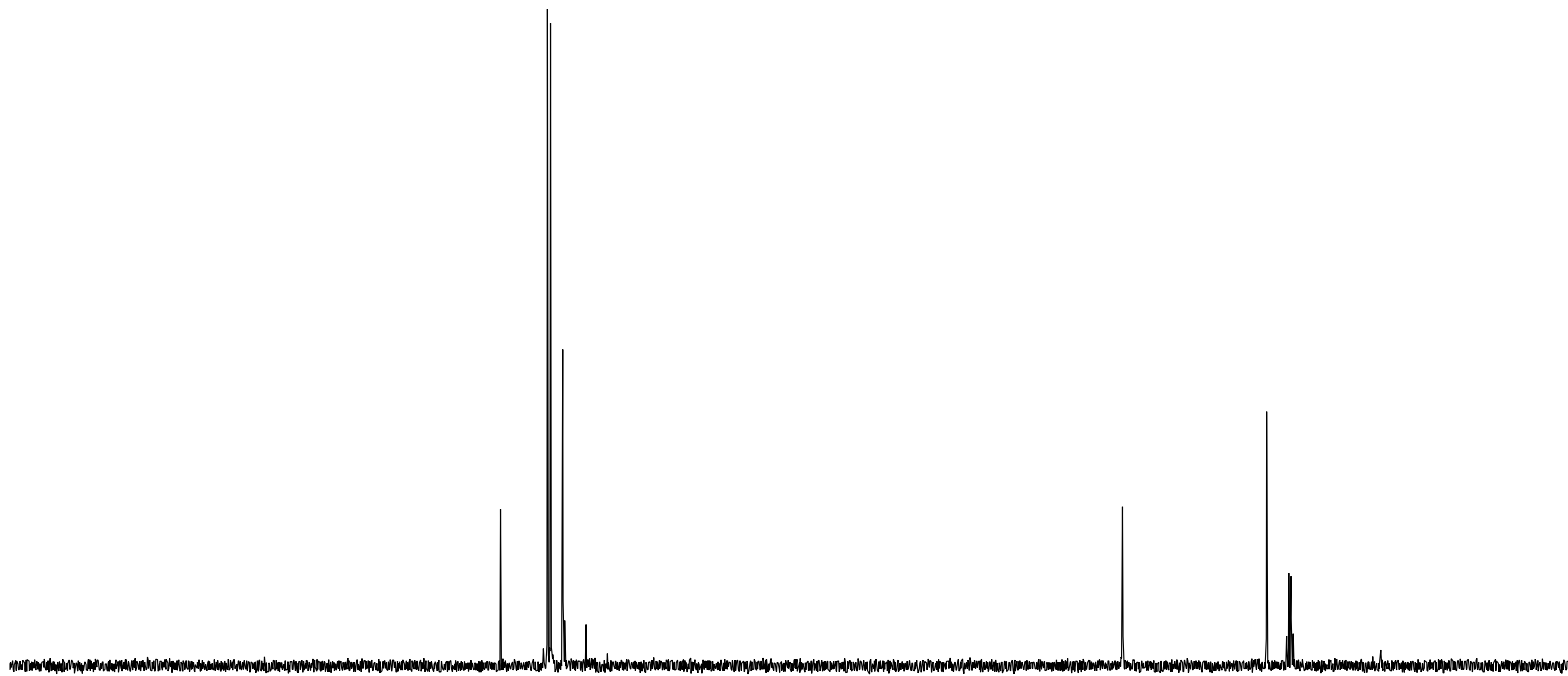

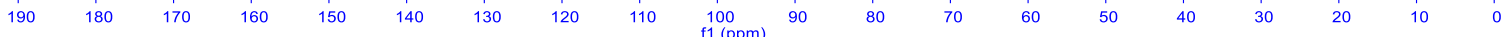




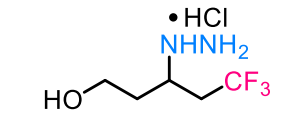

(4n, $400 \mathrm{MHz}, \mathrm{MeOH}-d_{4}$ )
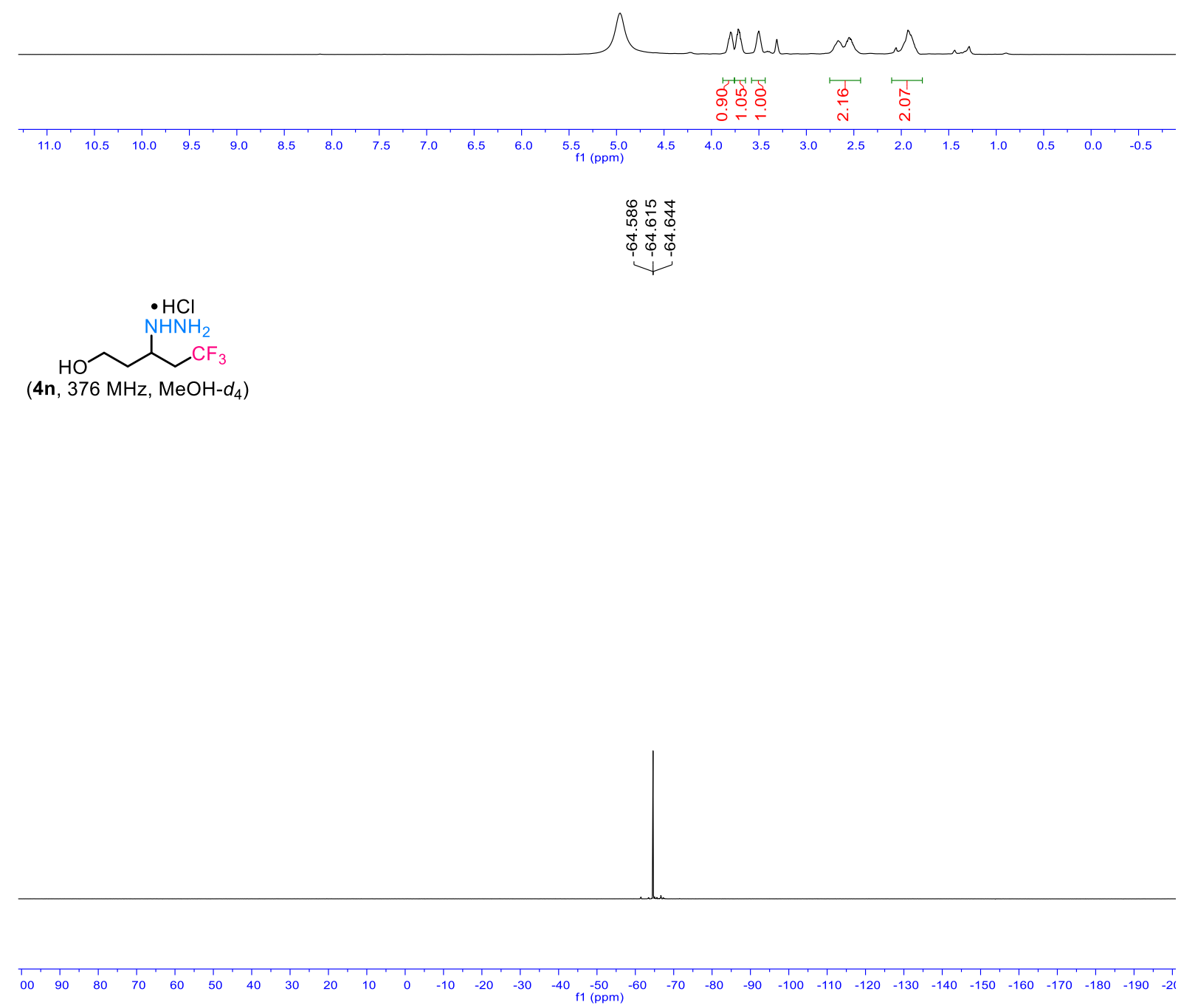

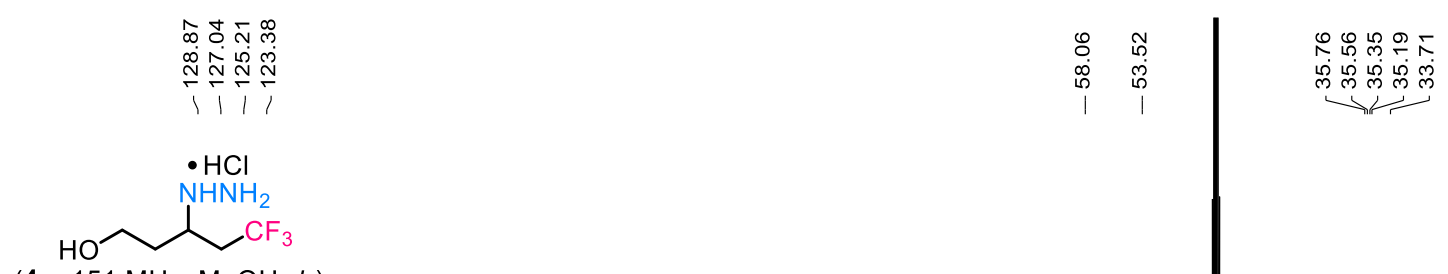

$\left(4 \mathrm{n}, 151 \mathrm{MHz}, \mathrm{MeOH}-d_{4}\right)$
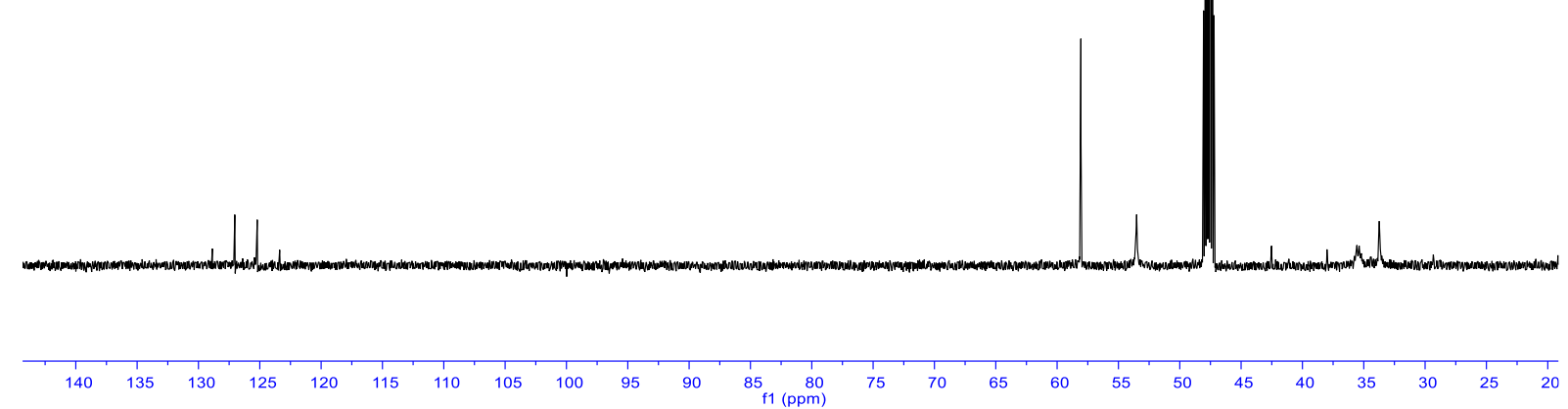

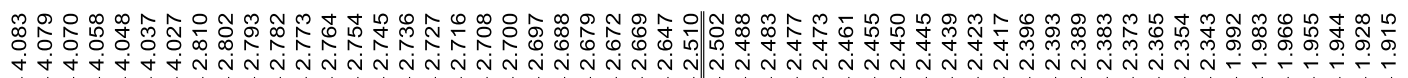

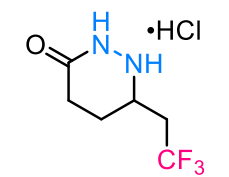

(4o, $\left.400 \mathrm{MHz}, \mathrm{D}_{2} \mathrm{O}\right)$

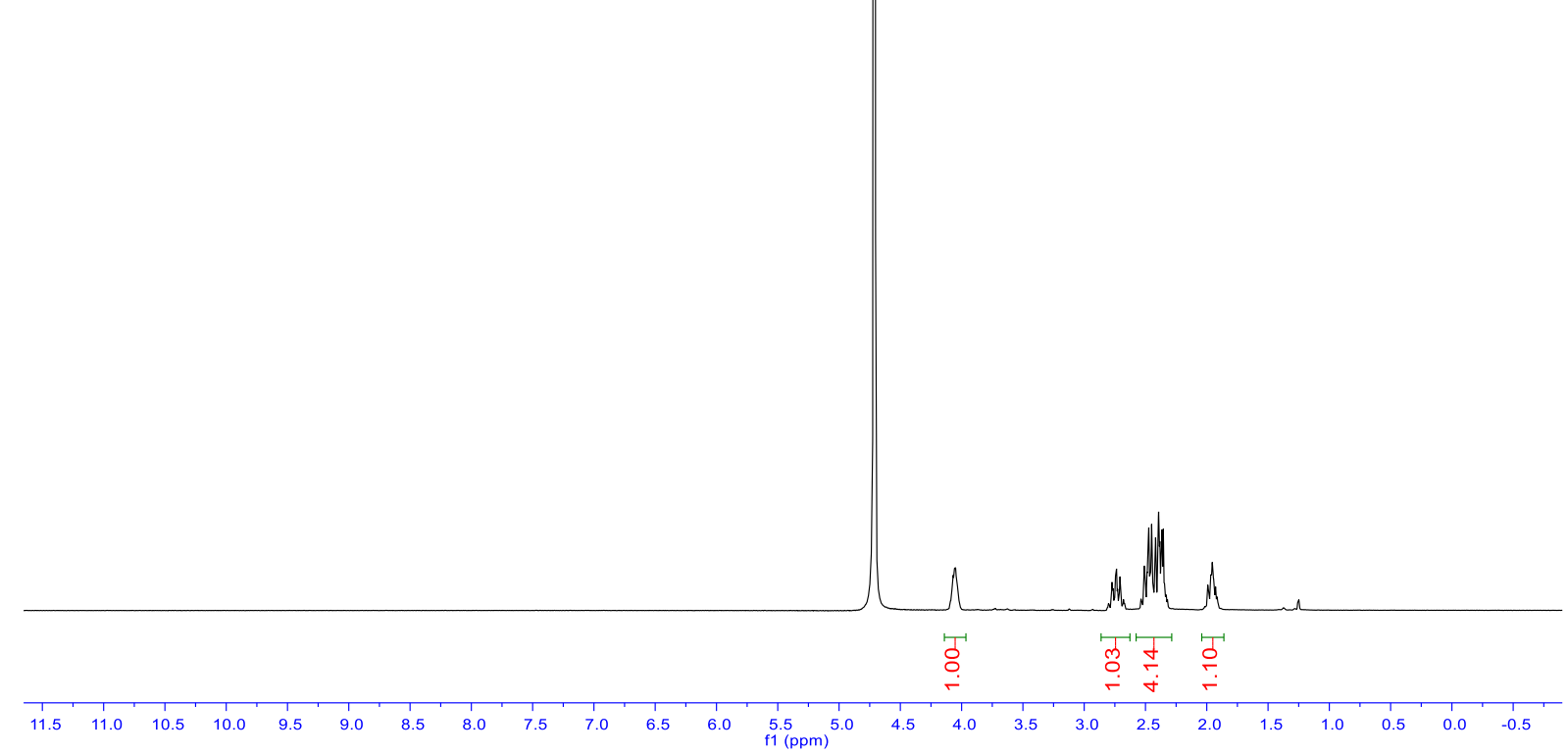




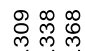

फั कृष

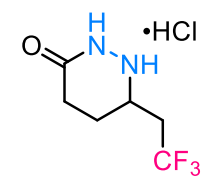

(4o, $\left.376 \mathrm{MHz}, \mathrm{D}_{2} \mathrm{O}\right)$

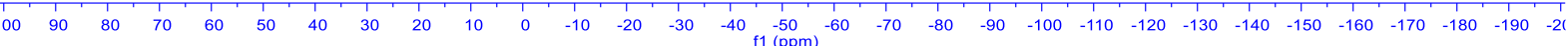

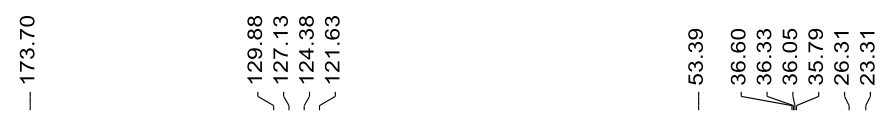

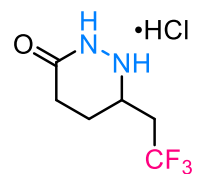

(4o, $101 \mathrm{MHz}, \mathrm{D}_{2} \mathrm{O}$ )

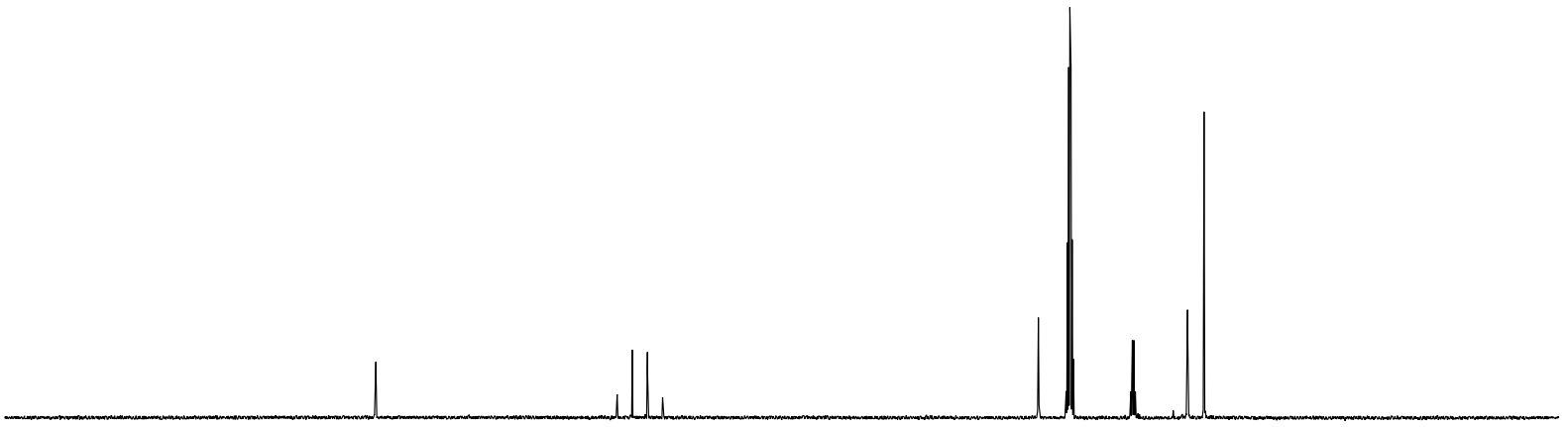

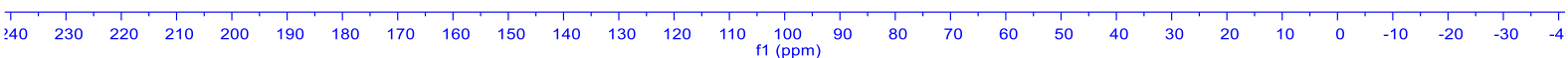


- $\mathrm{HCl}$

$\mathrm{NHNH}_{2}$

4p, $\left.400 \mathrm{MHz}, \mathrm{MeOH}-d_{4}\right)$

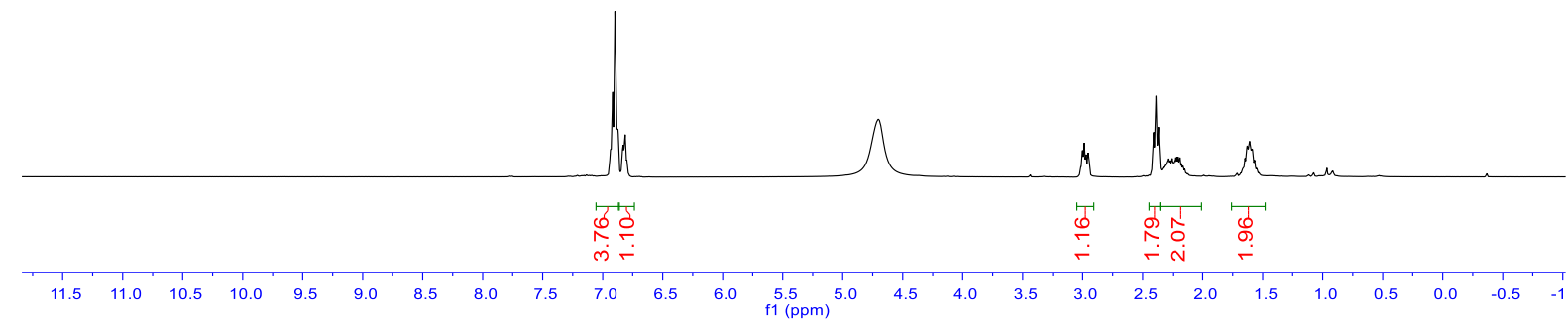

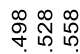

芦导导

- $\mathrm{HCl}$

$\mathrm{NHNH}_{2}$

$\mathrm{CF}_{3}$

(4p, $376 \mathrm{MHz}, \mathrm{MeOH}-d_{4}$ )

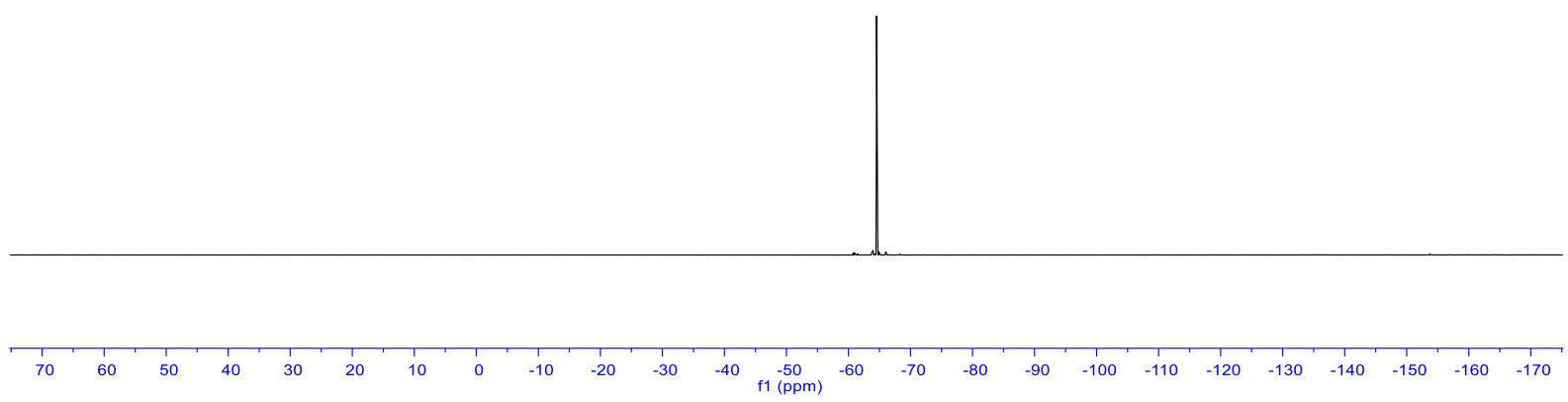




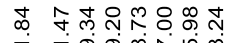

宁

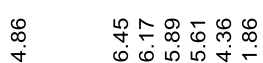

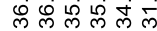

$\bullet \mathrm{HCl}$

$\mathrm{NHNH}_{2}$

$\sim \mathrm{CF}_{3}$

(4p, $101 \mathrm{MHz}, \mathrm{MeOH}-d_{4}$ )

$\left(4 \mathrm{p}, 101 \mathrm{MHz}, \mathrm{MeOH}-\mathrm{d}_{4}\right)$

(1)
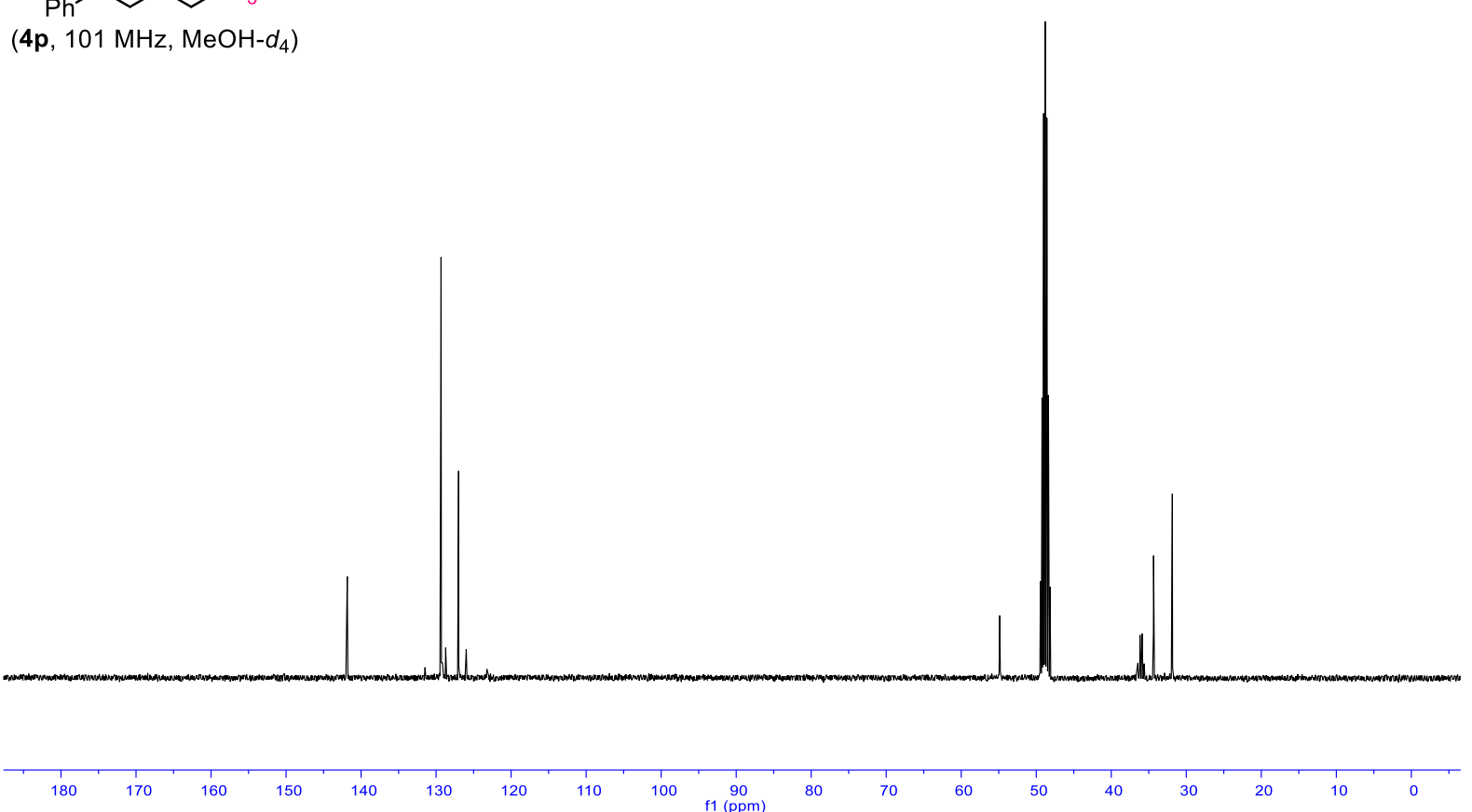

150

140

120

90
$\mathrm{f} 1(\mathrm{ppm})$

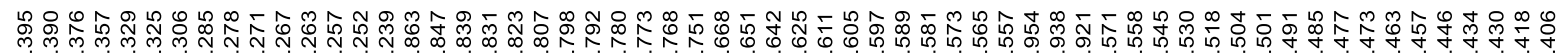

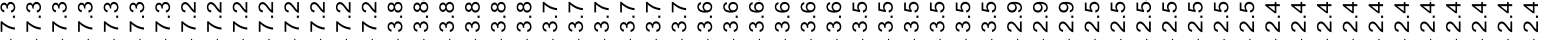<smiles>NC(Cl)CC(F)COCCc1ccccc1</smiles>

(4q, $400 \mathrm{MHz}, \mathrm{D}_{2} \mathrm{O}$ )

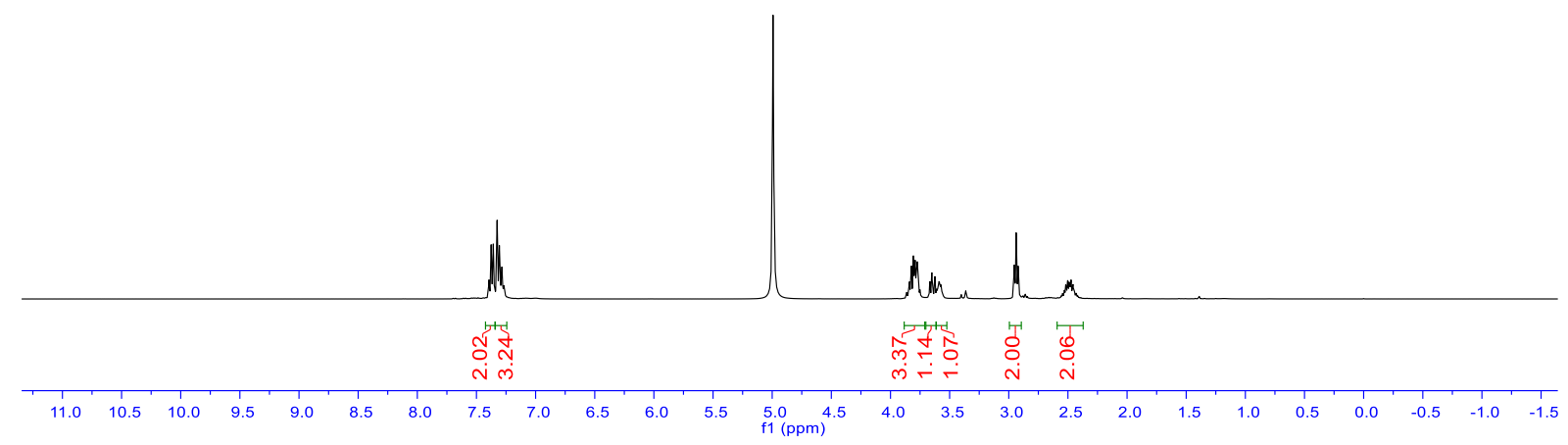




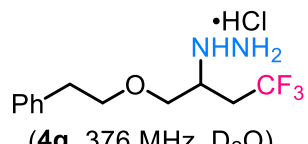

卓

(4q, $376 \mathrm{MHz}, \mathrm{D}_{2} \mathrm{O}$ )

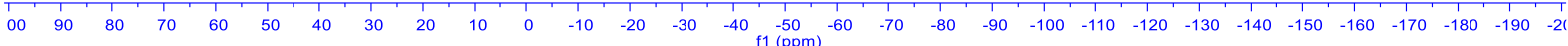

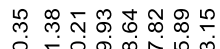

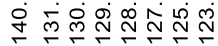

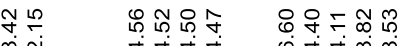

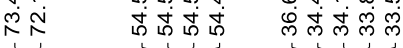

।

$1 / \mathrm{W}$

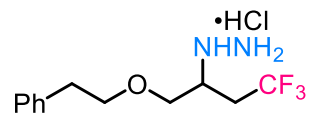

(4q, $101 \mathrm{MHz}, \mathrm{D}_{2} \mathrm{O}$ )
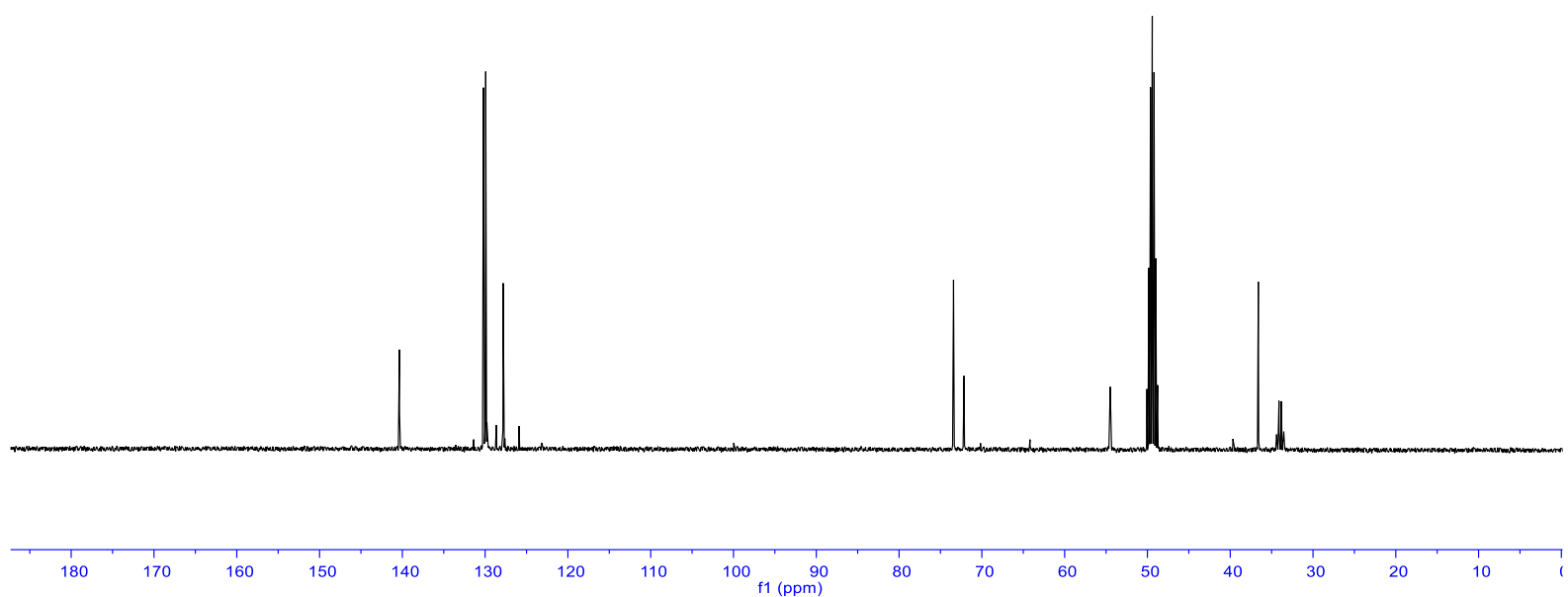


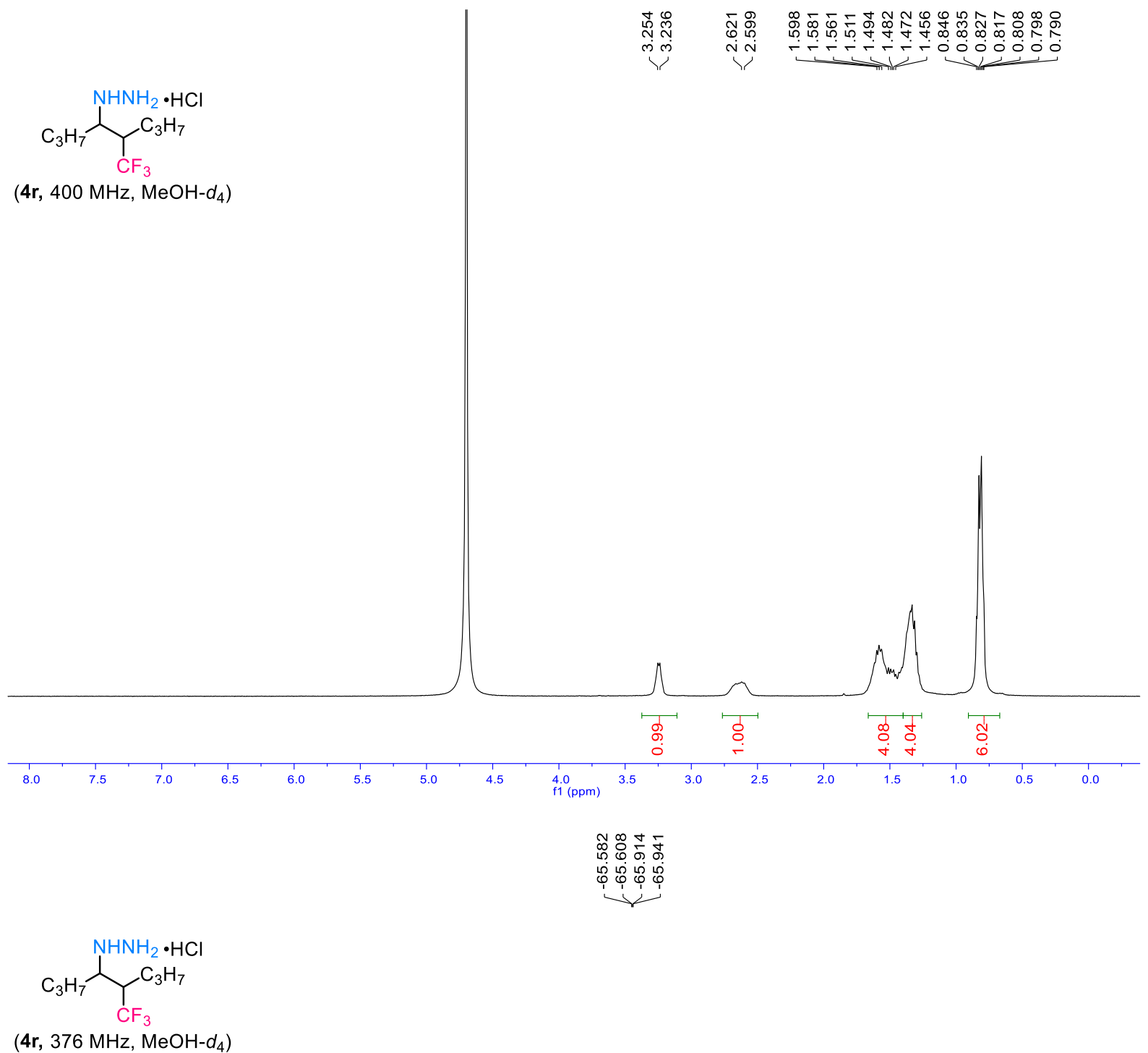

$\left(4 \mathrm{r}, 376 \mathrm{MHz}, \mathrm{MeOH}-d_{4}\right)$

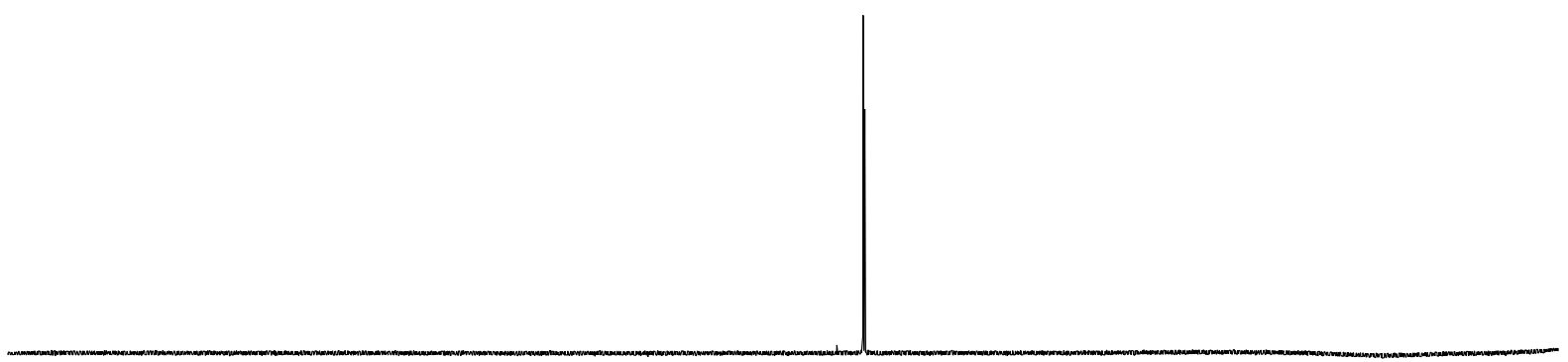

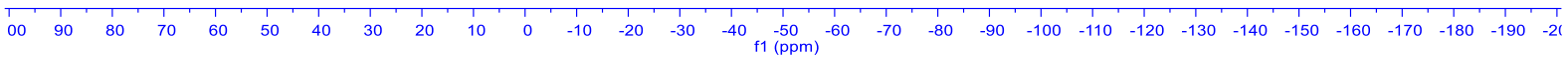




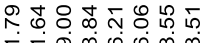

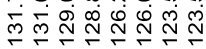

प्रा

$\mathrm{NHNH}_{2} \cdot \mathrm{HCl}$

$\mathrm{C}_{3} \mathrm{H}_{7} \bigodot_{\mathrm{CF}_{3}}^{\mathrm{C}_{3} \mathrm{H}_{7}}$

(4r, $\left.101 \mathrm{MHz}, \mathrm{D}_{2} \mathrm{O}\right)$

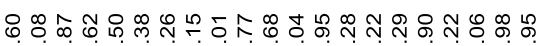

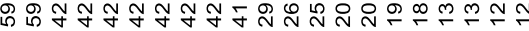
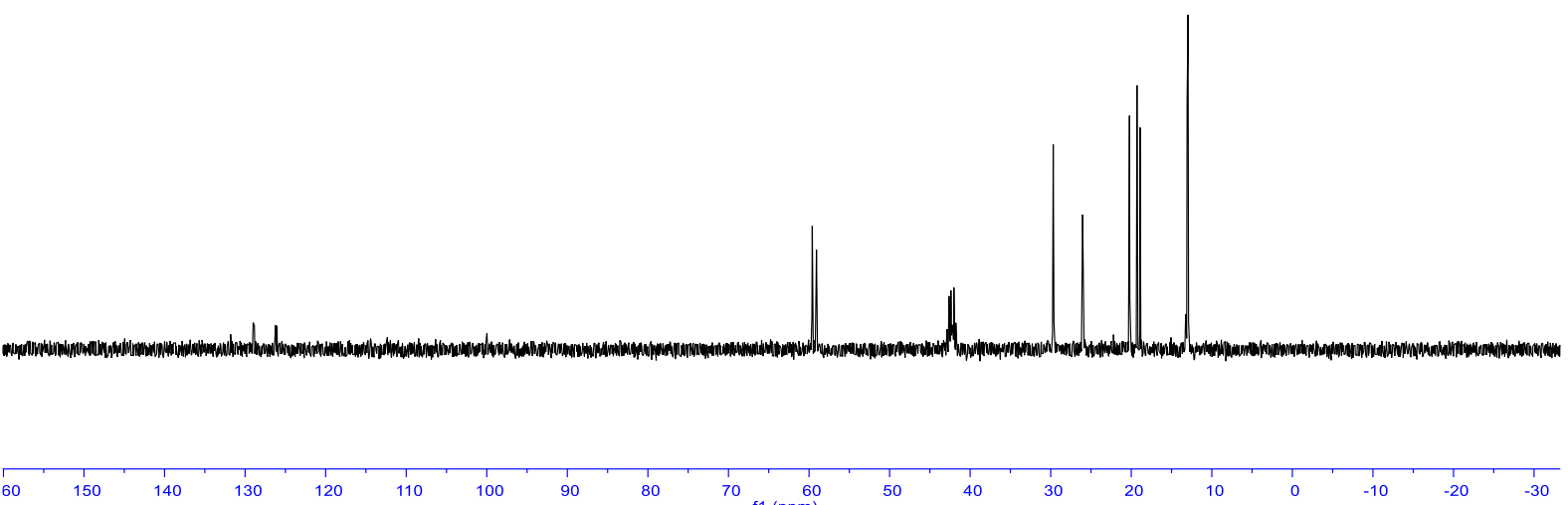

f1 $\begin{array}{r}60 \\ \text { ppm) }\end{array}$

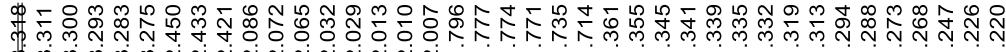

$\cdot \mathrm{HC}$

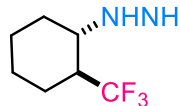

(4s, $600 \mathrm{MHz}, \mathrm{D}_{2} \mathrm{O}$ )

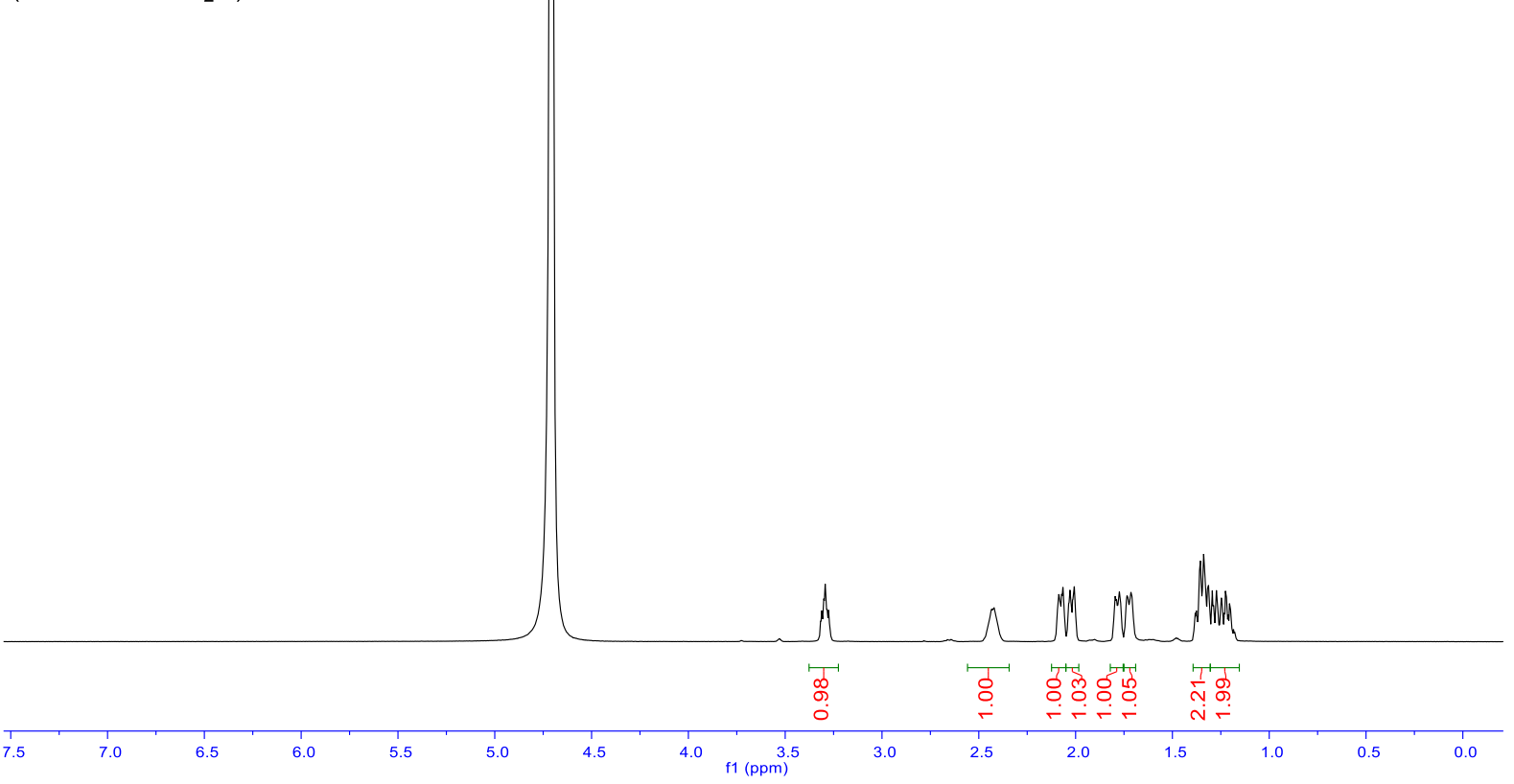


$\bullet \mathrm{HCl}$

$\overbrace{\mathrm{CF}_{3}}{ }^{\mathrm{NHNH}}$

(4s, $376 \mathrm{MHz}, \mathrm{D}_{2} \mathrm{O}$ )

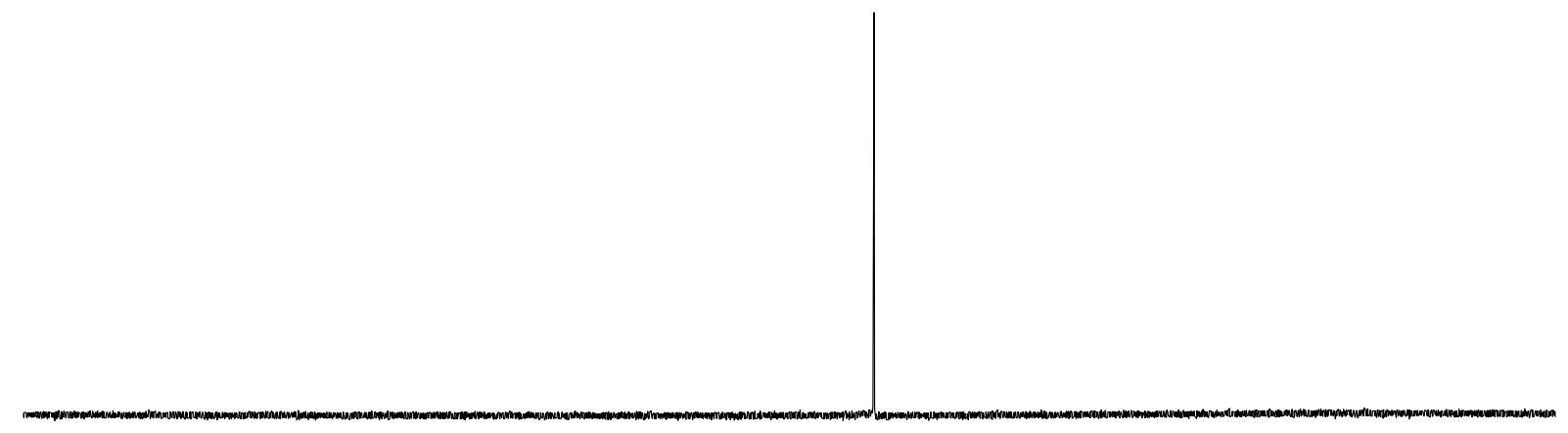

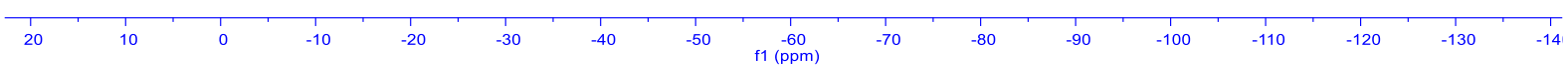

ำ ㄱำ

mํํำ

1111

งู

m

$\cdot \mathrm{HCl}$

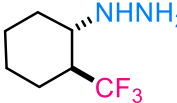

(4s, $\left.101 \mathrm{MHz}, \mathrm{MeOH}-d_{4}\right)$

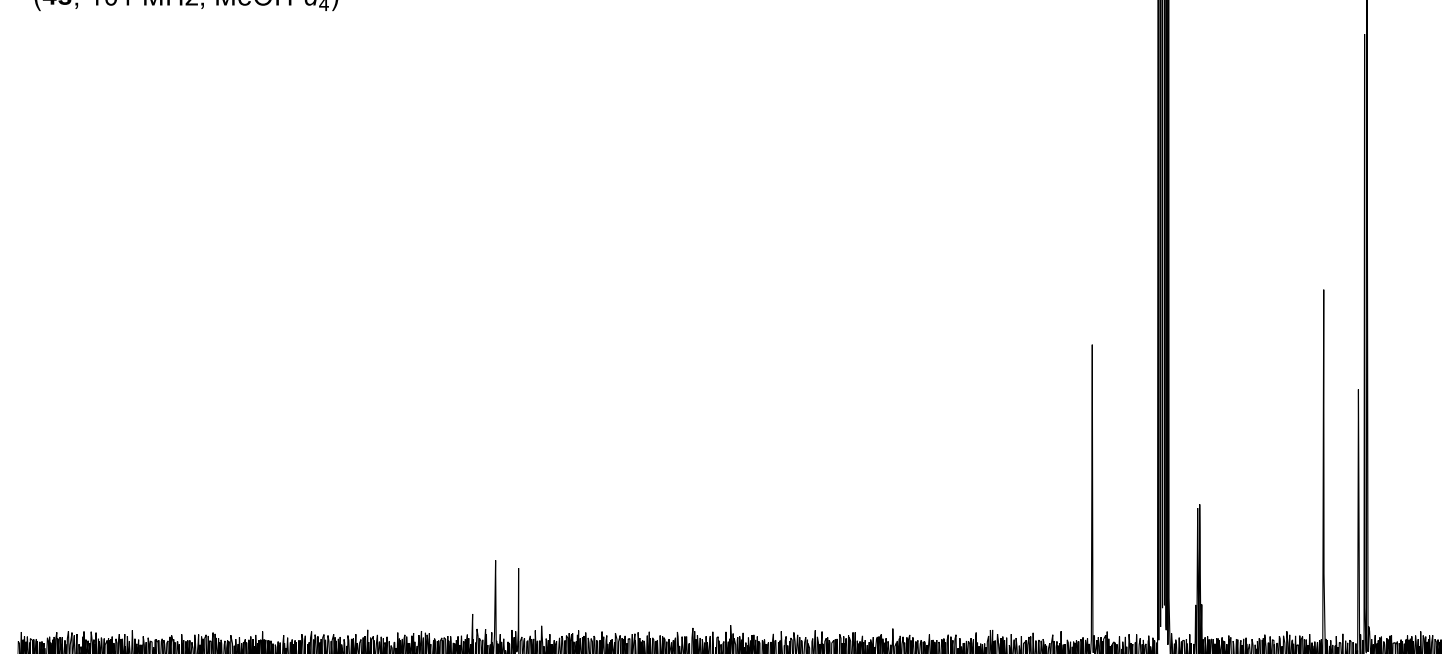

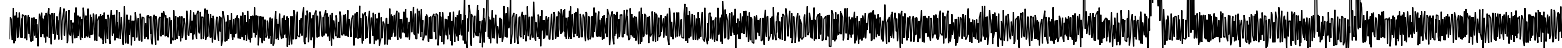

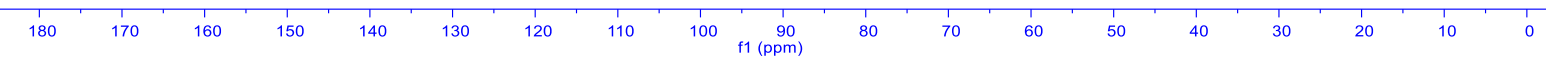




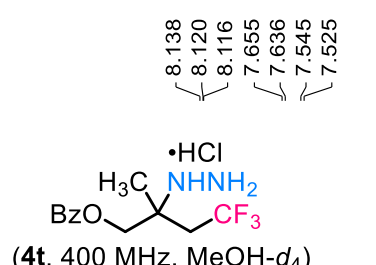

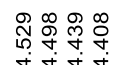

ก่ง

$\sqrt{\sin 2}$

(4t, $400 \mathrm{MHz}, \mathrm{MeOH}-d_{4}$ )

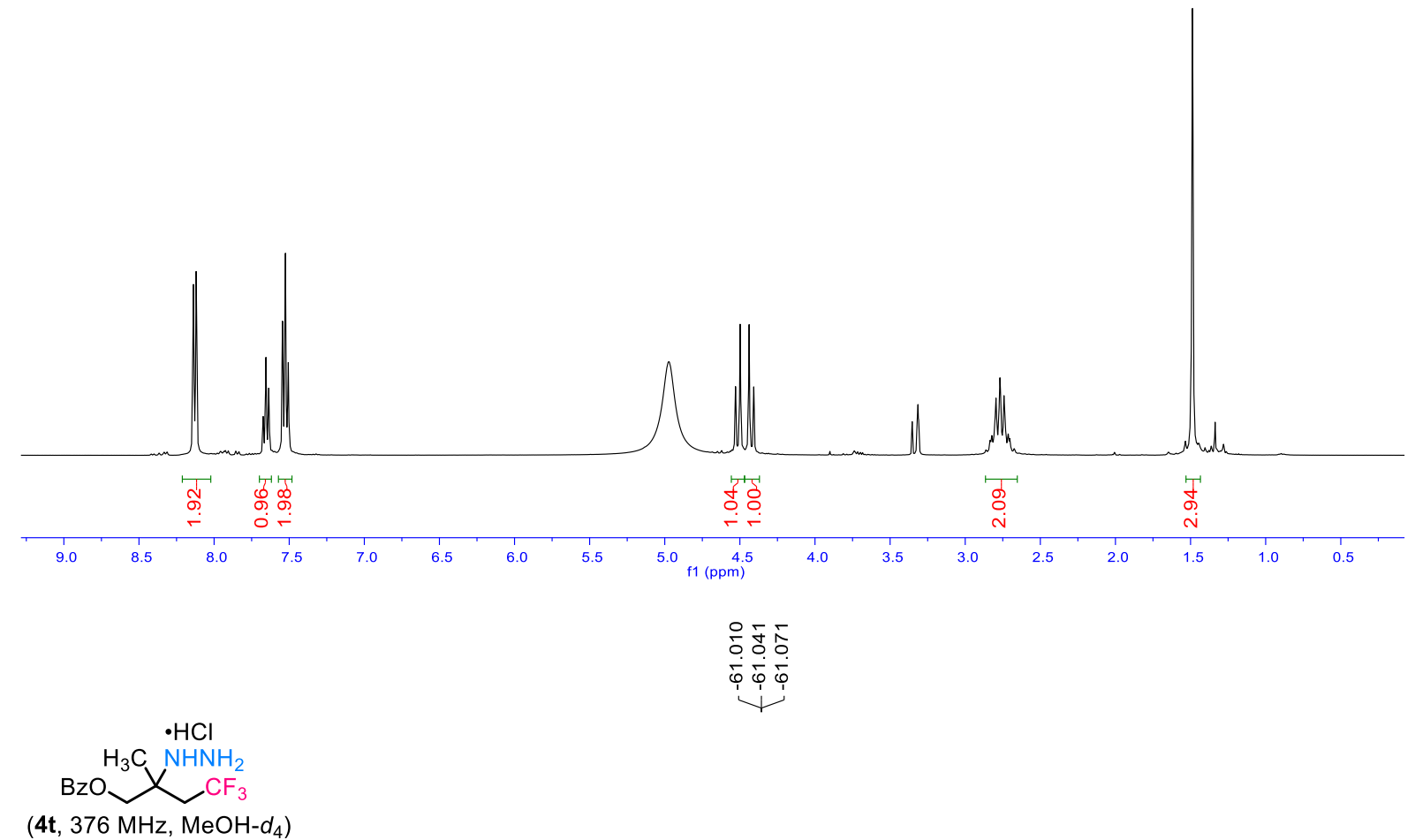

(4t, $376 \mathrm{MHz}, \mathrm{MeOH}-d_{4}$ )

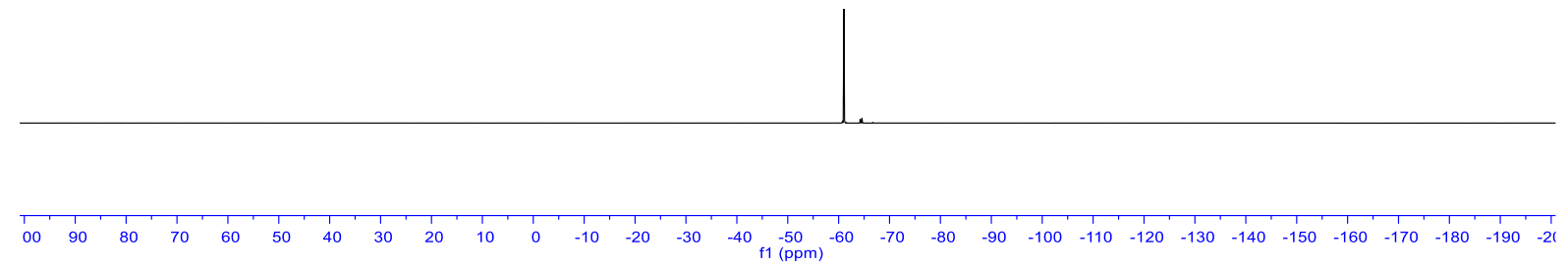



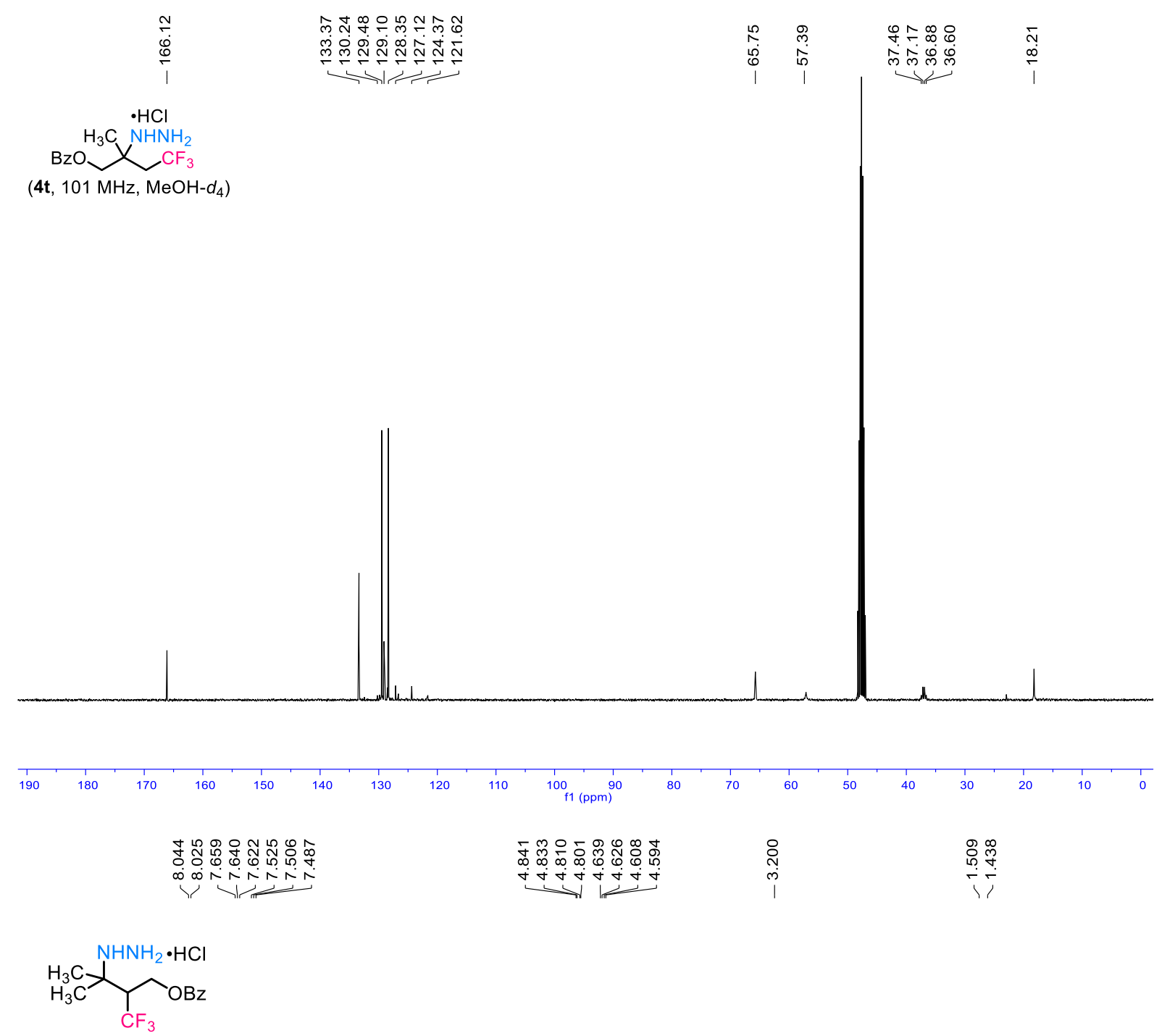

(4u, $\left.400 \mathrm{MHz}, \mathrm{MeOH}-d_{4}\right)$

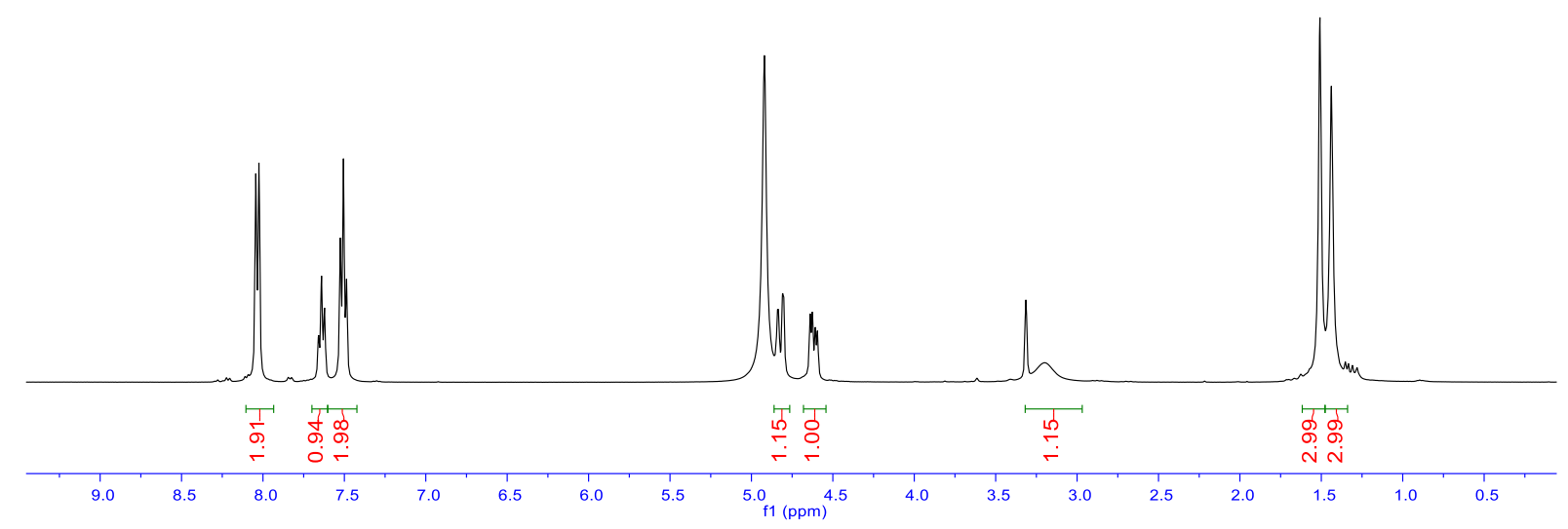




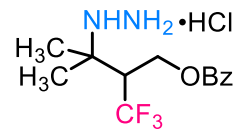

$\left(4 \mathbf{u}, 376 \mathrm{MHz}, \mathrm{MeOH}-d_{4}\right)$

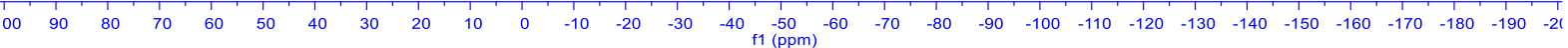

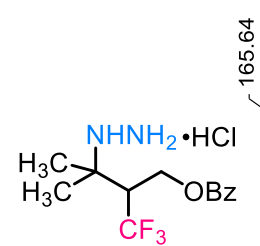

$\left(4 \mathbf{u}, 101 \mathrm{MHz}, \mathrm{MeOH}-d_{4}\right)$

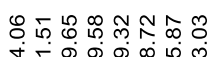

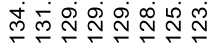

要

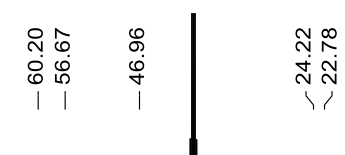

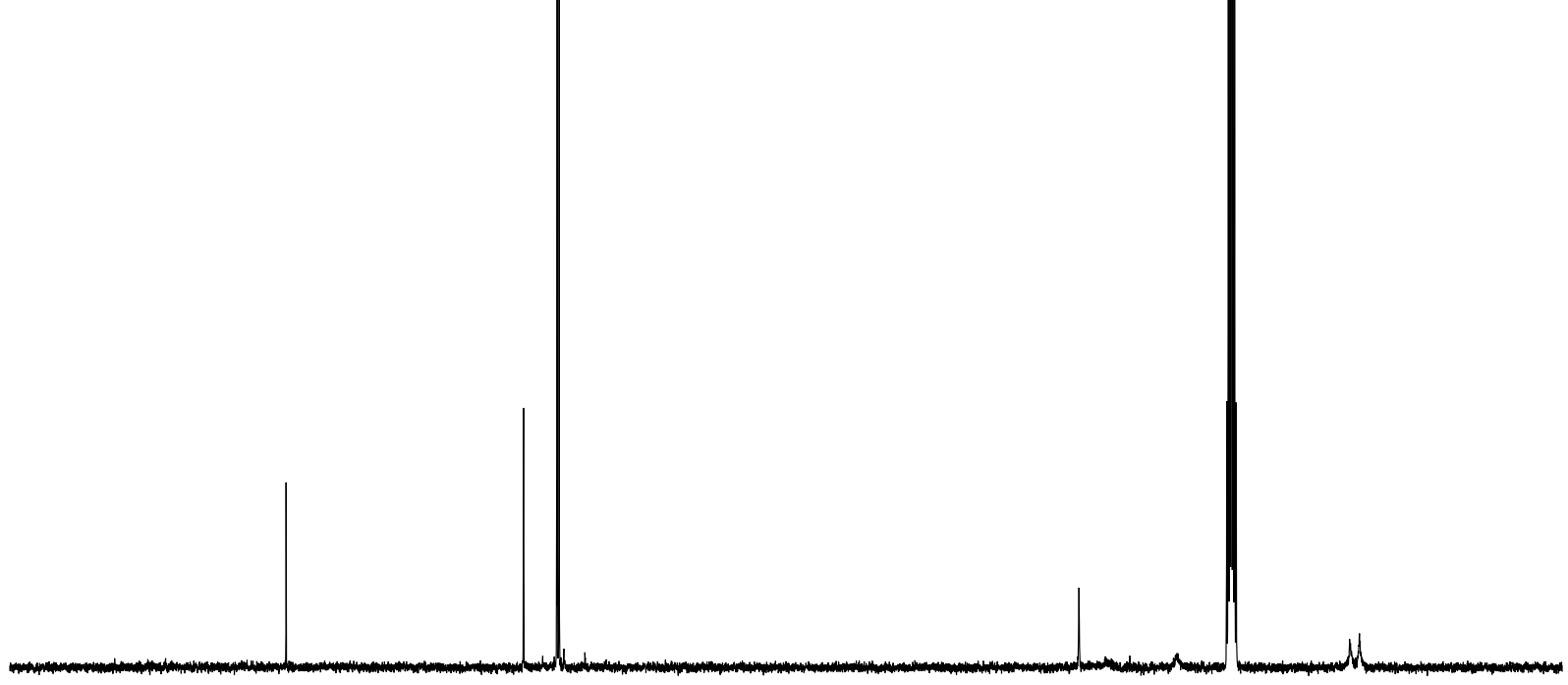

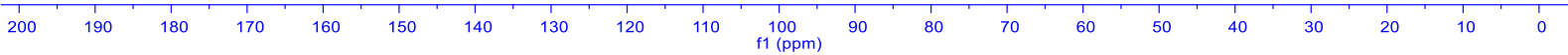


<smiles>CCOC(=O)/C(O)=C1\CCc2ccccc2C1=O</smiles>

$\left(5,400 \mathrm{MHz}, \mathrm{CDCl}_{3}\right)$

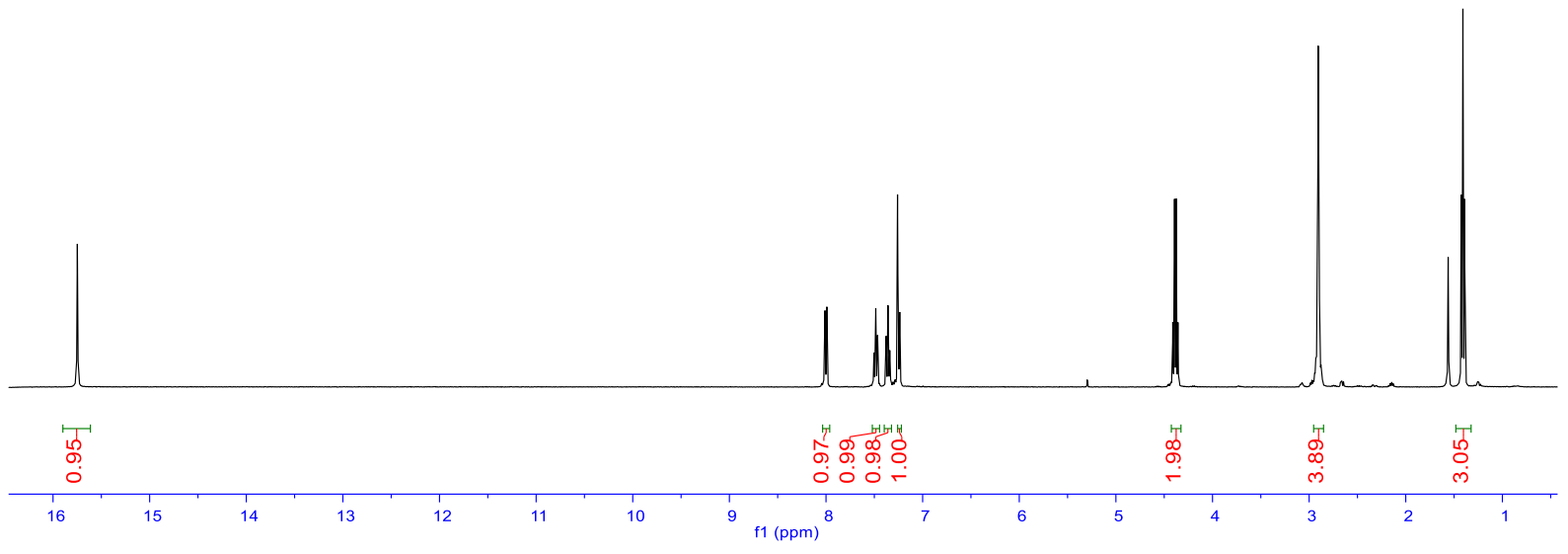

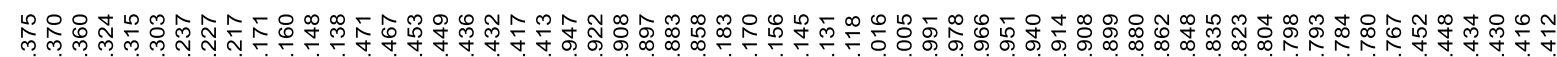

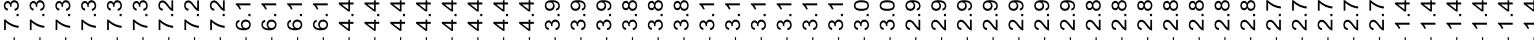<smiles>CCOC(=O)c1nn(C(CC(F)(F)F)c2ccccc2)c2c1CCc1ccccc1-2</smiles>

$\left(6,400 \mathrm{MHz}, \mathrm{CDCl}_{3}\right)$

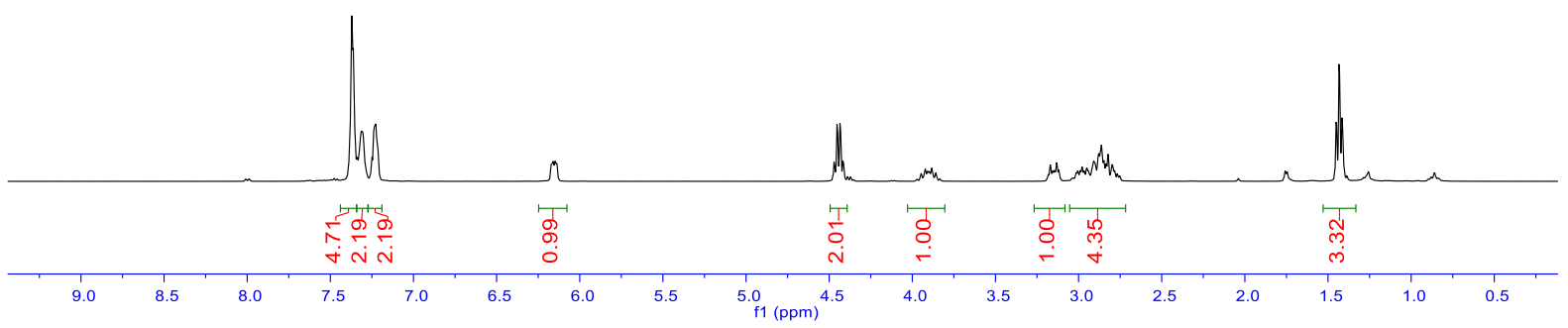




\section{옹ํำ}

过宅

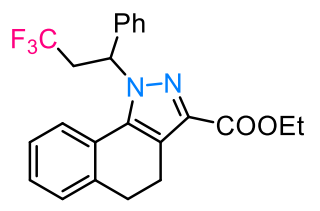

(6, $376 \mathrm{MHz}, \mathrm{CDCl}_{3}$ )
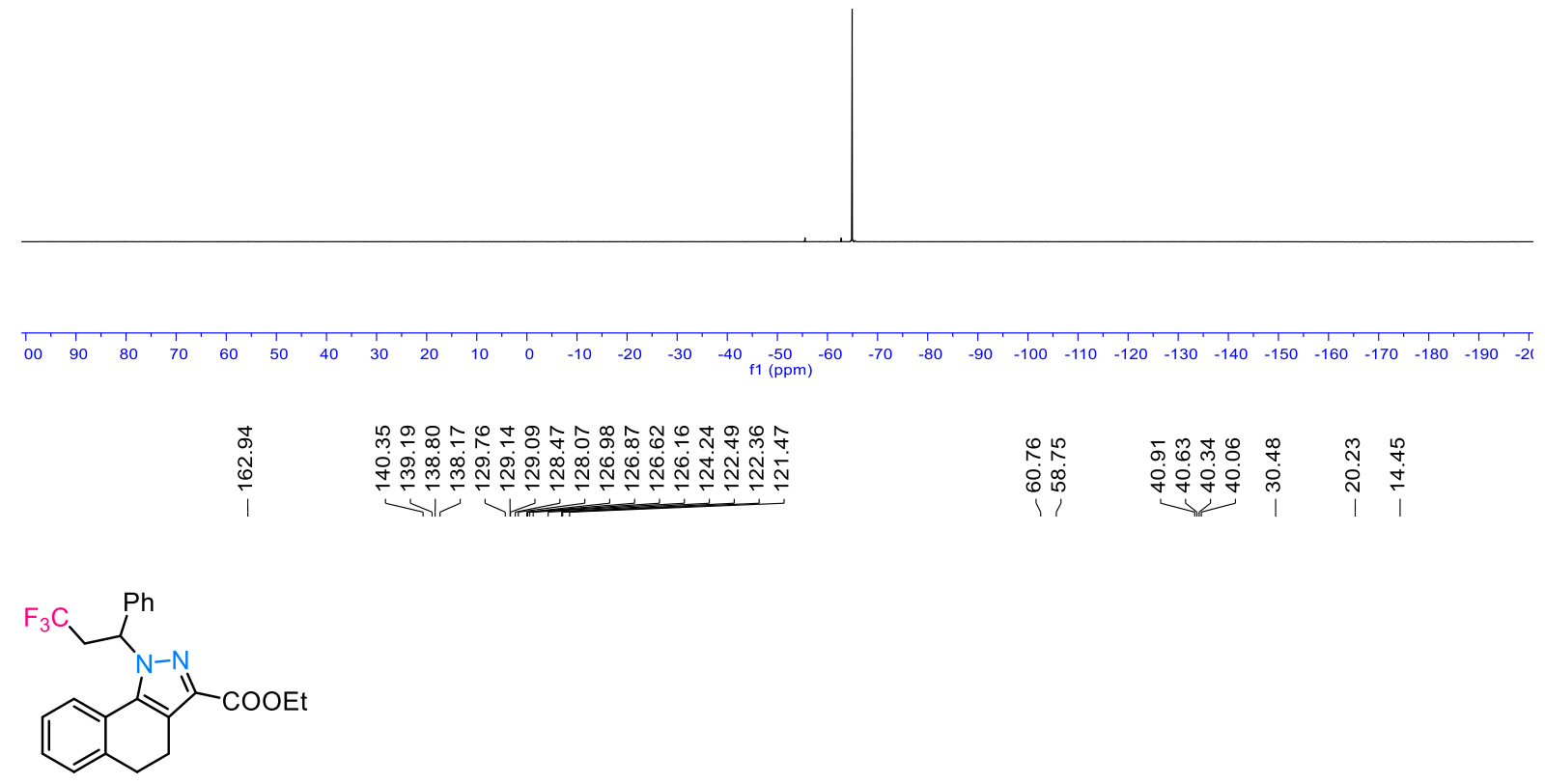

(6, $101 \mathrm{MHz}, \mathrm{CDCl}_{3}$ )
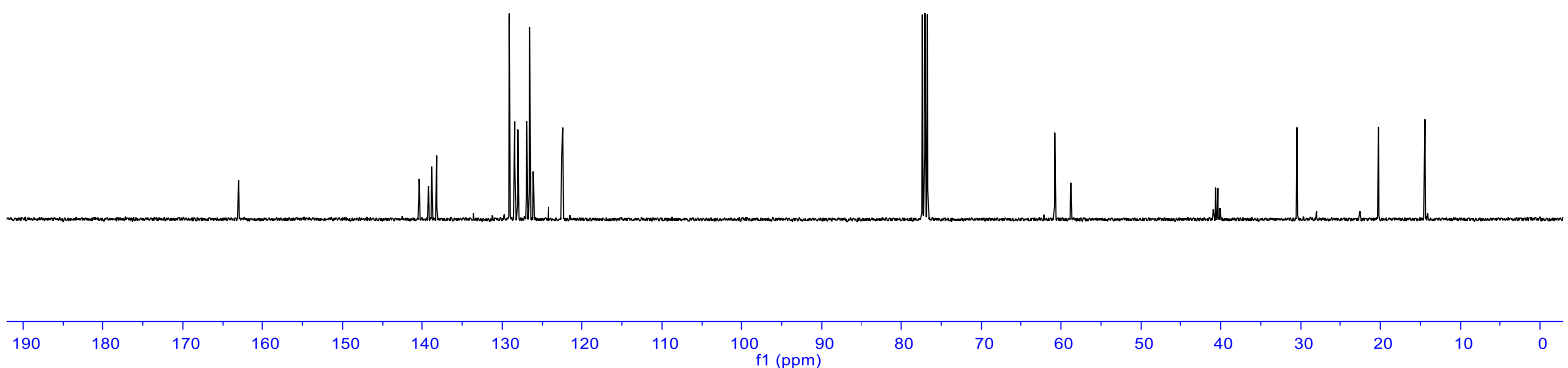

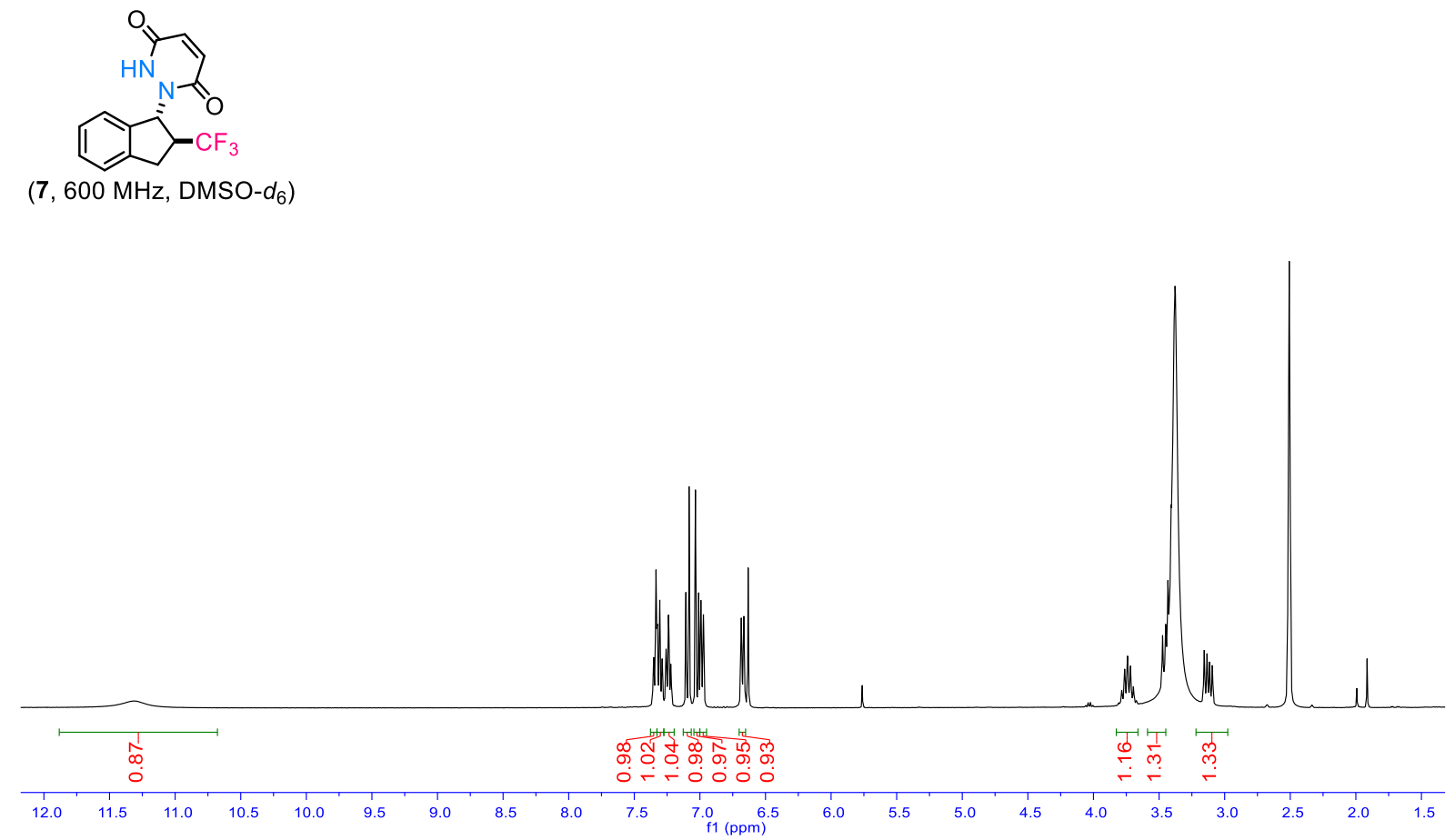

กิ่

कृष

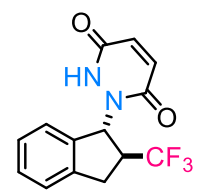

$\left(7,565 \mathrm{MHz}, \mathrm{DMSO}-d_{6}\right)$

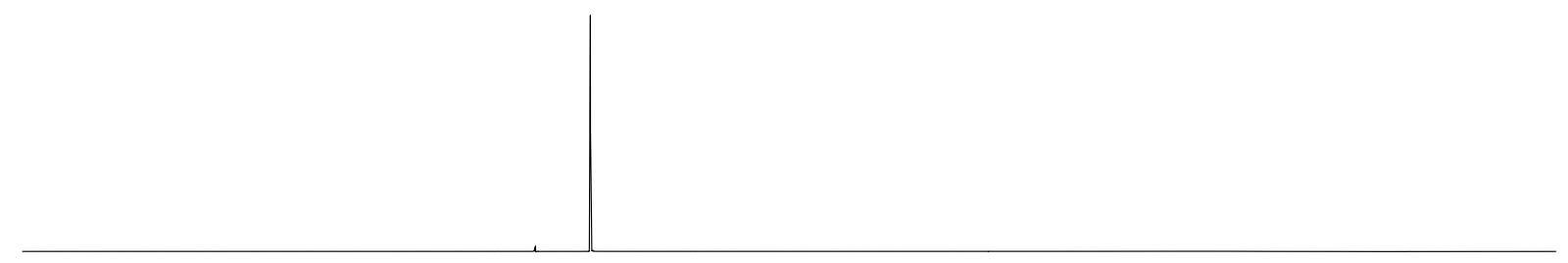



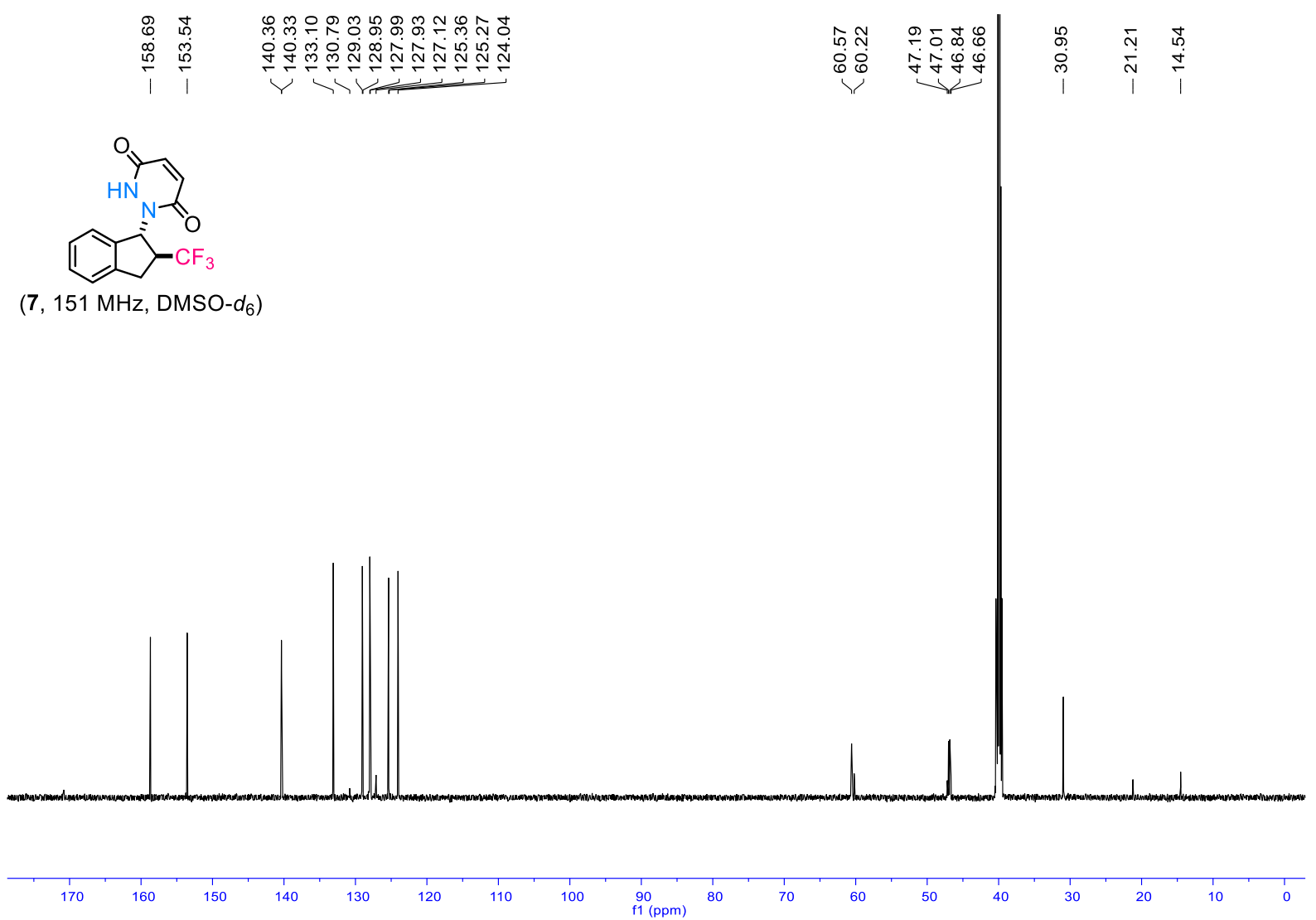

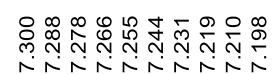

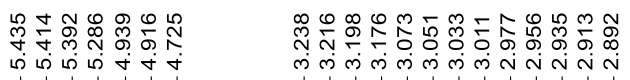

rNnNañán

il i

m n m n

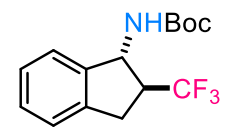

(8, $400 \mathrm{MHz}, \mathrm{CDCl}_{3}$ )

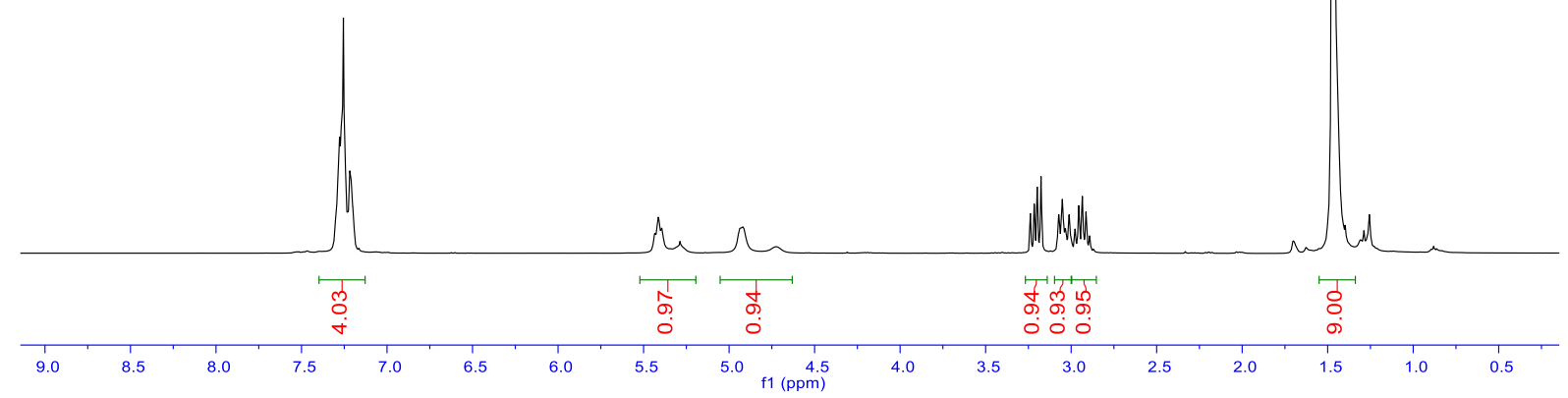




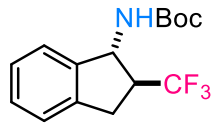

$\left(8,376 \mathrm{MHz}, \mathrm{CDCl}_{3}\right)$

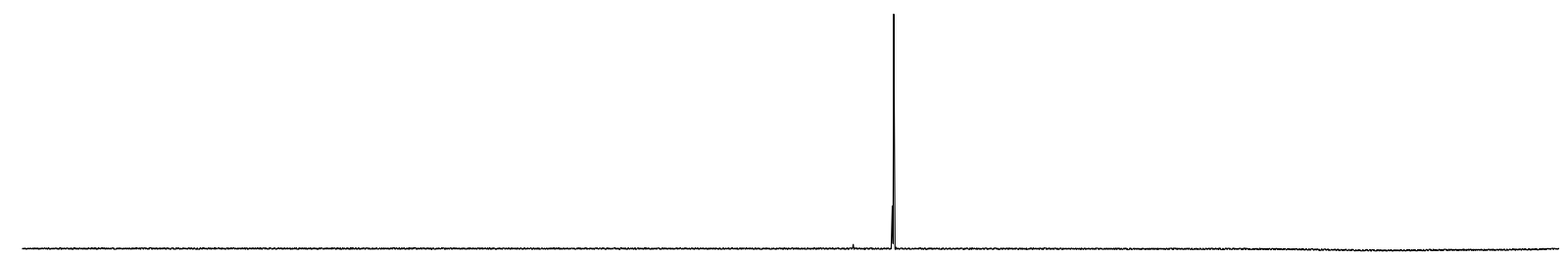

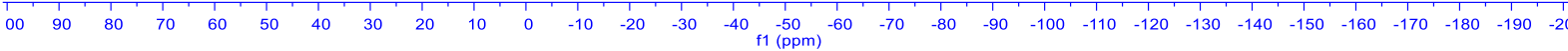

\begin{tabular}{|c|c|c|}
\hline 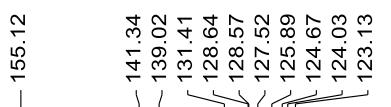 & $\begin{array}{l}8 \\
0 \\
\infty \\
1\end{array}$ & 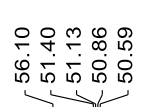 \\
\hline
\end{tabular}

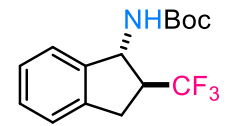

$\left(8,101 \mathrm{MHz}, \mathrm{CDCl}_{3}\right)$

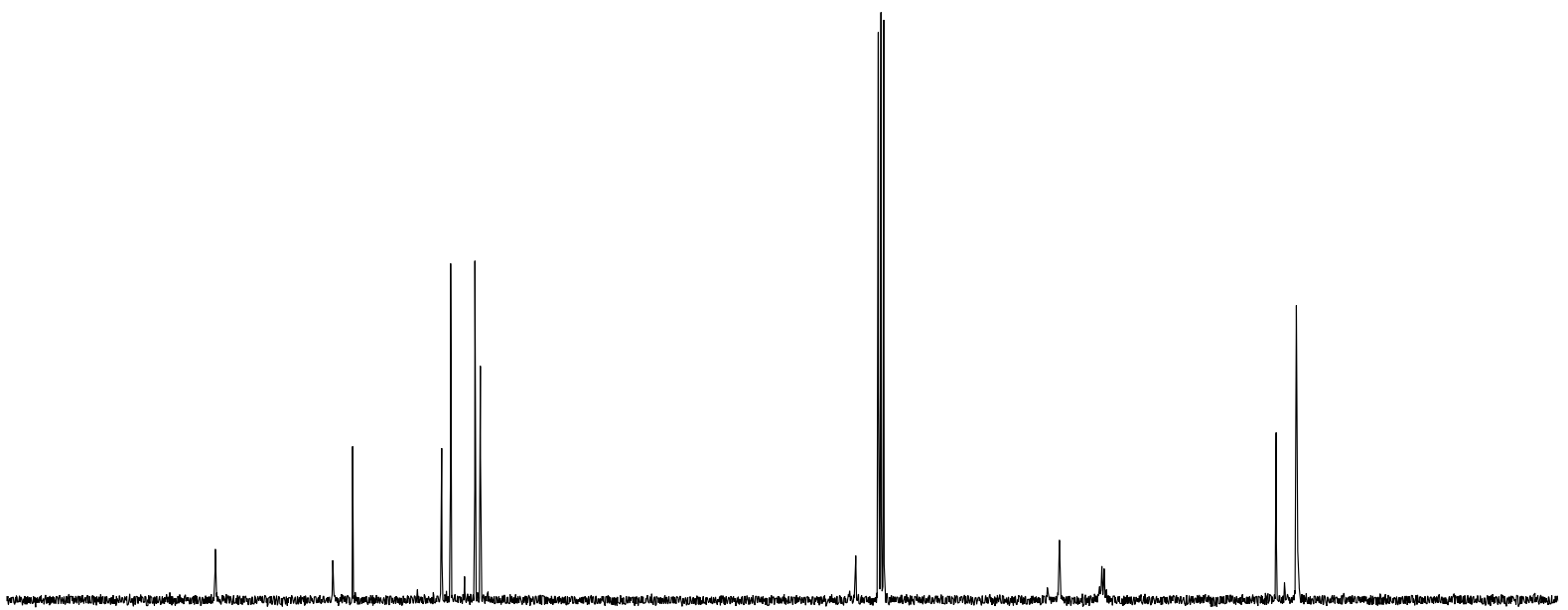

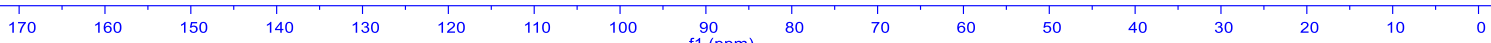




$$
\begin{gathered}
\mathrm{Boc}^{-\mathrm{N}}-\mathrm{SO}_{2} \mathrm{CF}_{3} \\
\left.\mathrm{BN}^{-\mathrm{Boc}}, 400 \mathrm{MHz}, \mathrm{CDCl}_{3}\right)
\end{gathered}
$$

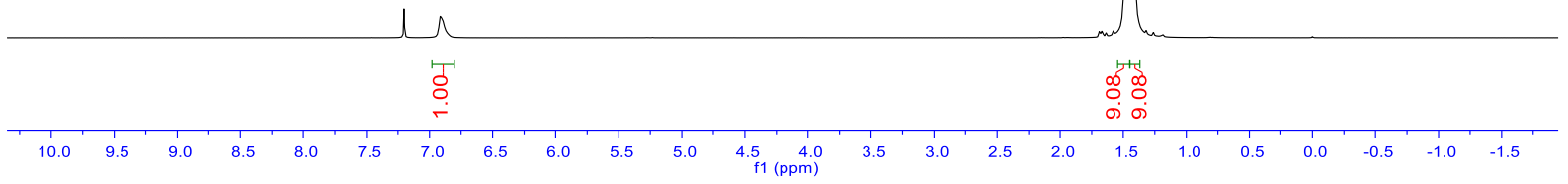

초ำ

$\sqrt{\sqrt{n}}$

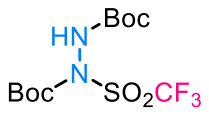

(9, $\left.376 \mathrm{MHz}, \mathrm{CDCl}_{3}\right)$

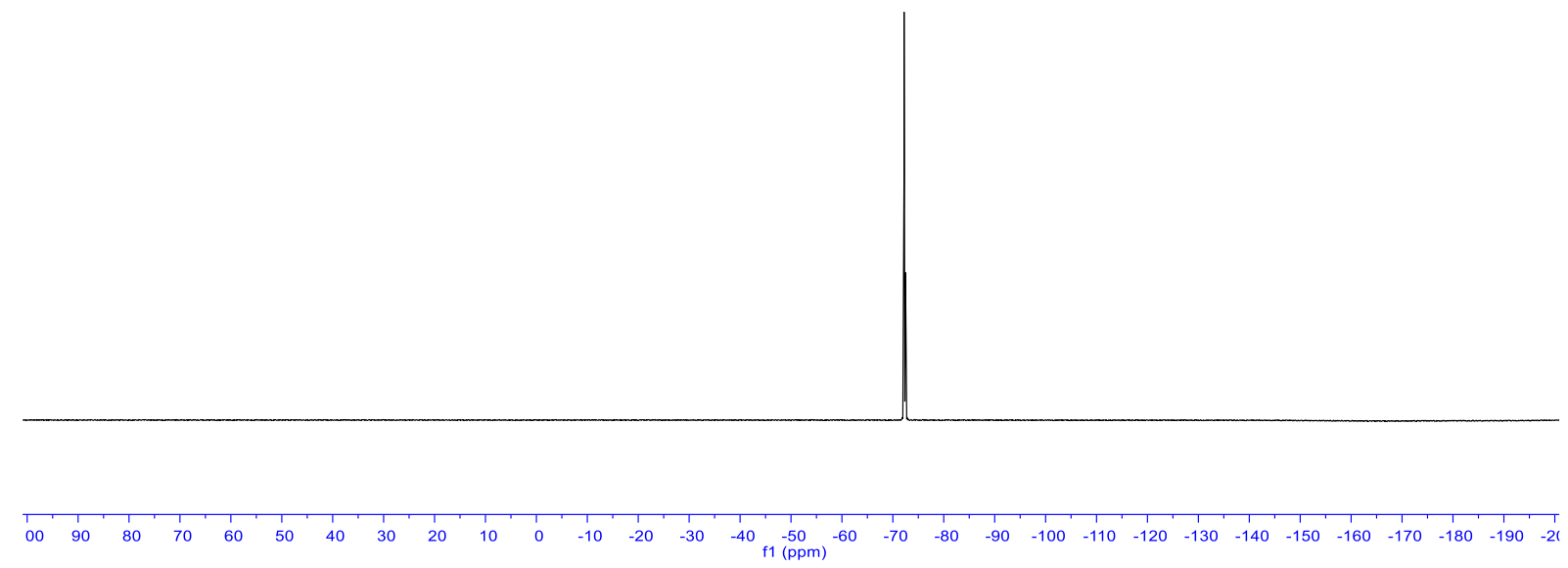




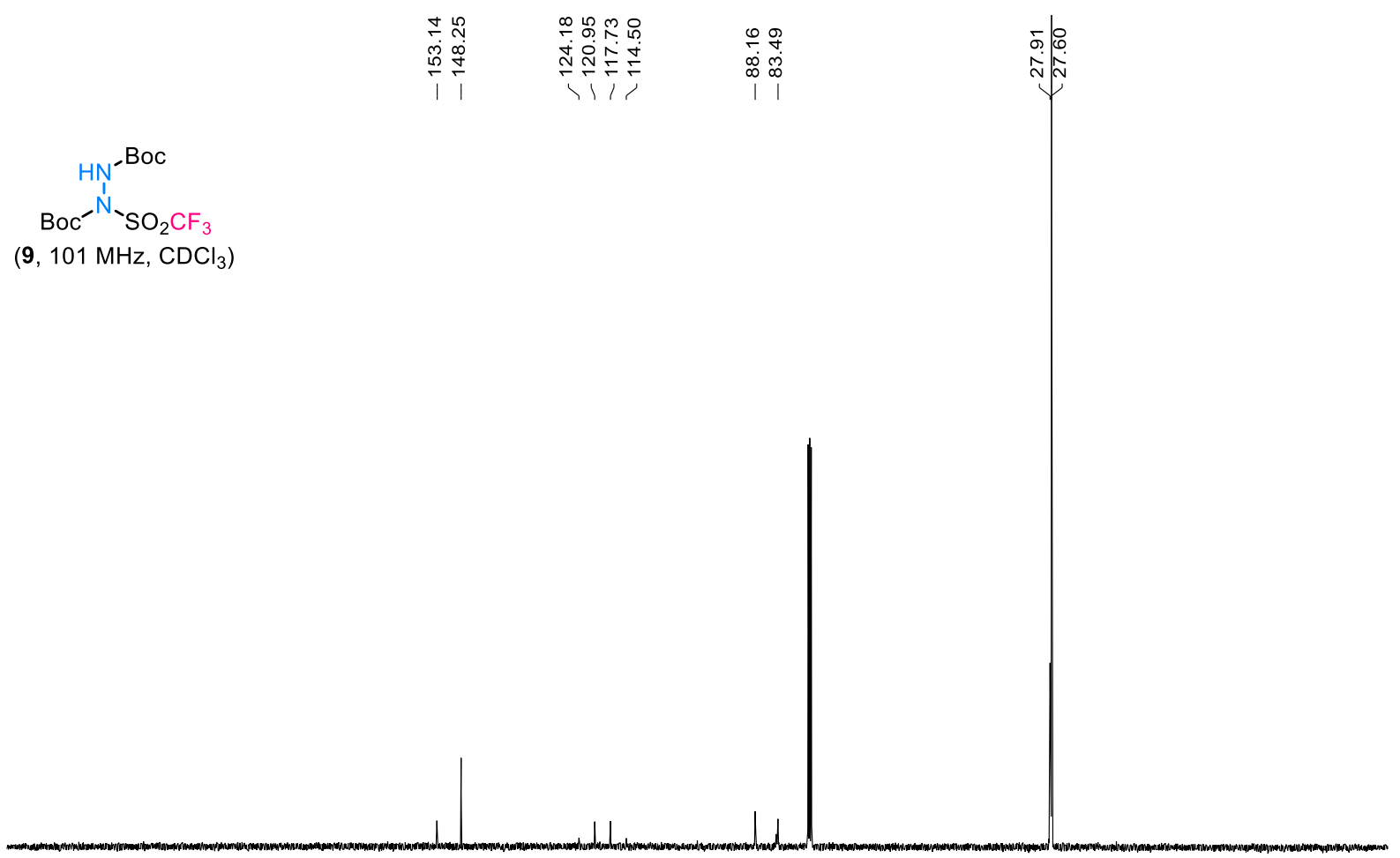

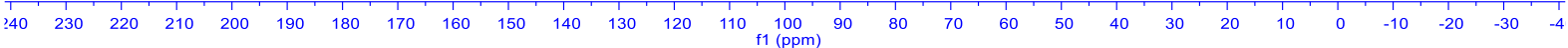

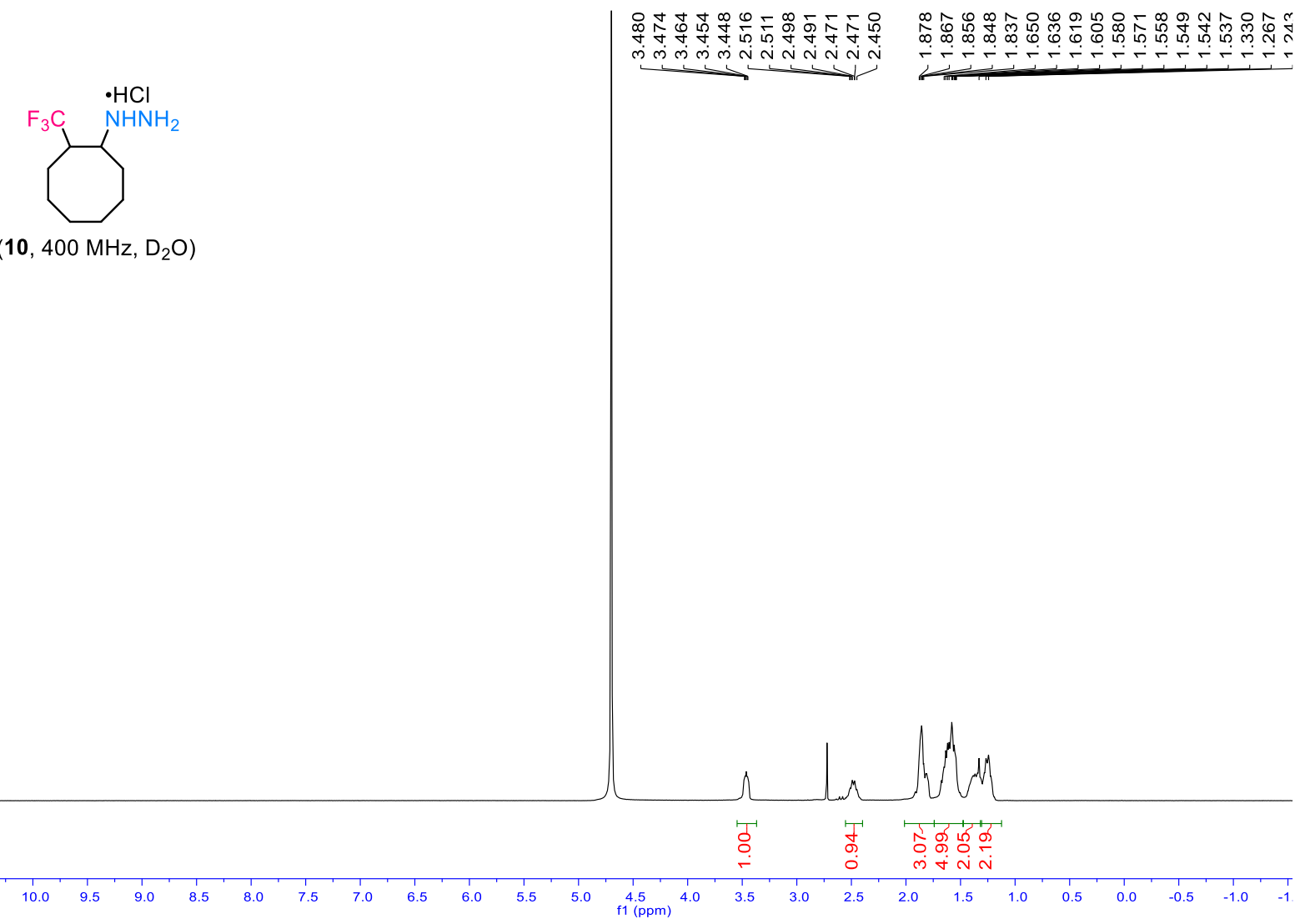




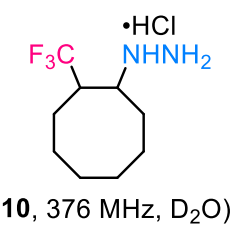

5
$m$
0
0
0
1
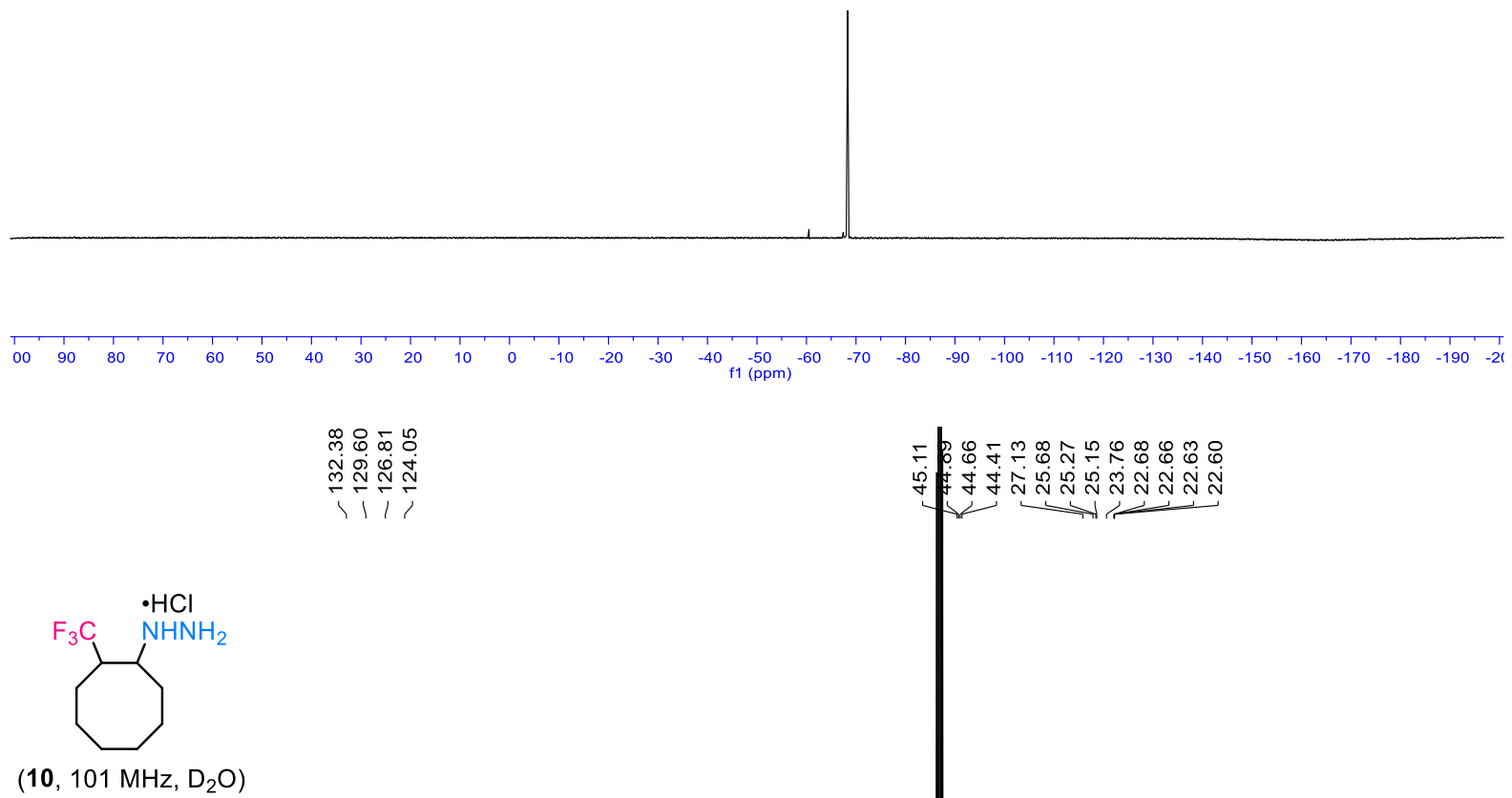

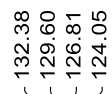

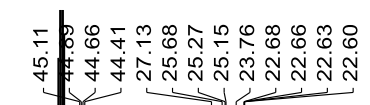

$\left(\mathbf{1 0}, 101 \mathrm{MHz}, \mathrm{D}_{2} \mathrm{O}\right)$

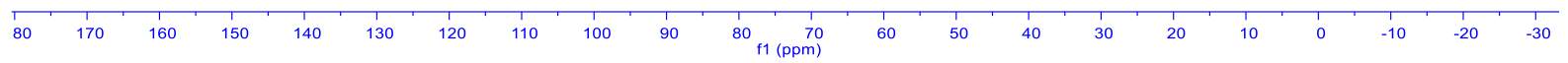

S66 


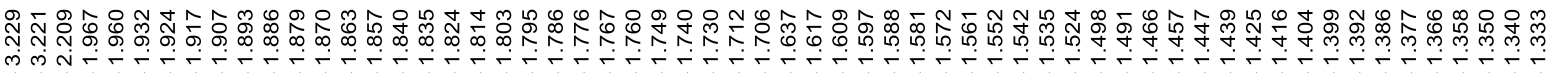<smiles>O=[Po]([O-])NC1CCCCC(C(F)(F)F)CC1NO</smiles>

(11, $400 \mathrm{MHz}, \mathrm{D}_{2} \mathrm{O}$ )

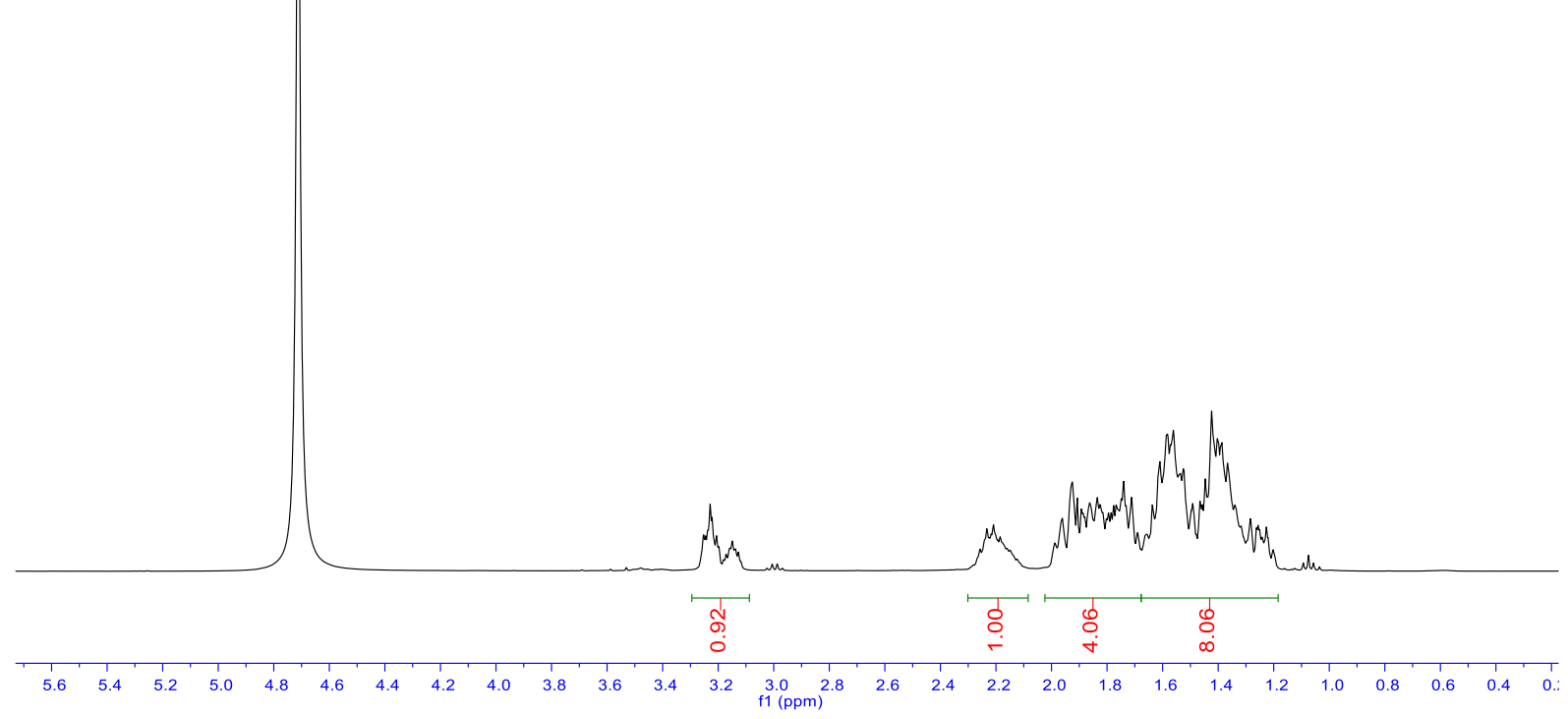

نَّ

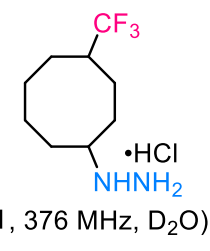

(11, $\left.376 \mathrm{MHz}, \mathrm{D}_{2} \mathrm{O}\right)$

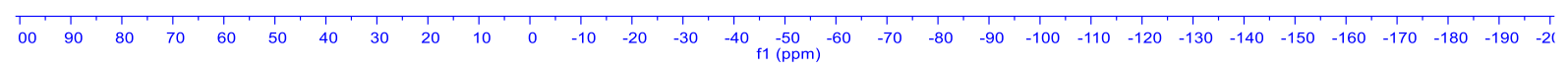

S67 


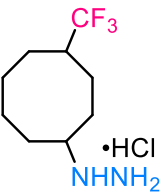

(11, $\left.101 \mathrm{MHz}, \mathrm{D}_{2} \mathrm{O}\right)$
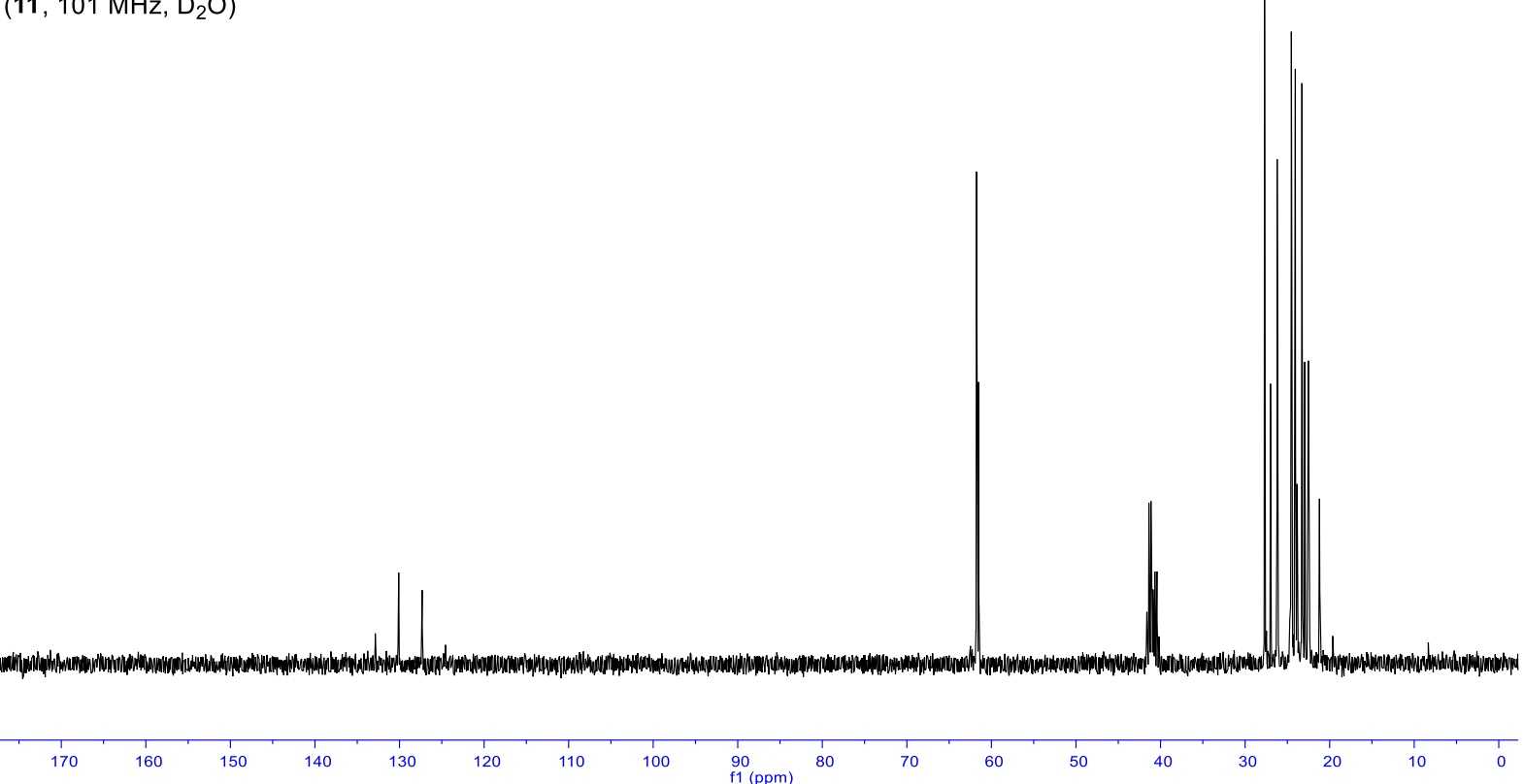

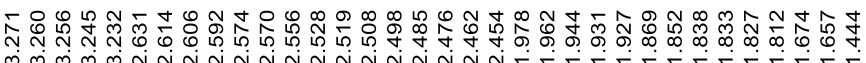

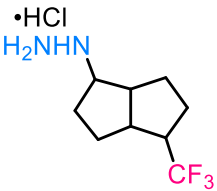

(12, isomer $1,400 \mathrm{MHz}, \mathrm{D}_{2} \mathrm{O}$ )

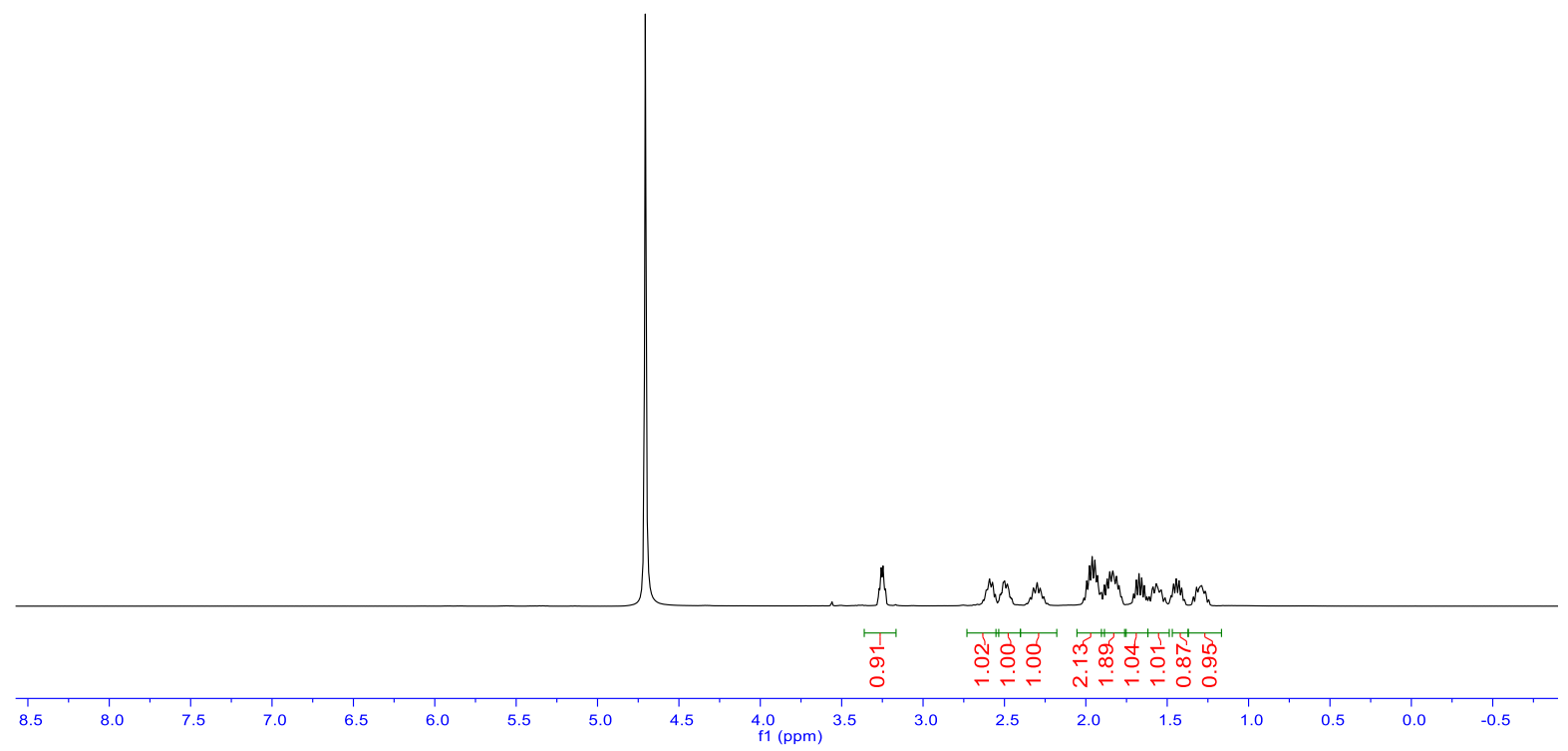


$\cdot \mathrm{HCl}$

$\bigcup_{\mathrm{CF}_{3}}$

(12, isomer $1,376 \mathrm{MHz}, \mathrm{D}_{2} \mathrm{O}$ )

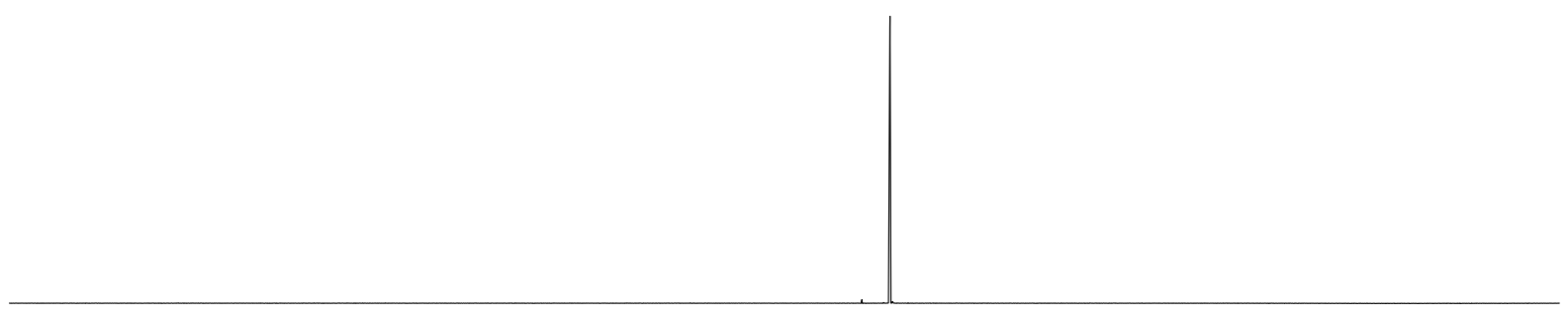

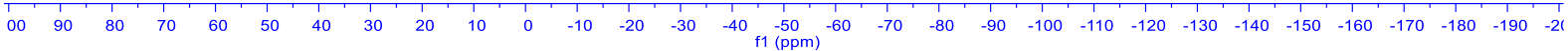

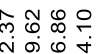

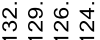
กิ
रा1

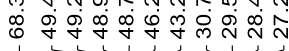

$\cdot \mathrm{HCl}$

$\mathrm{H}_{2} \mathrm{NHN}$

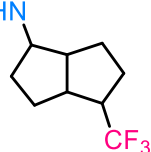

(12, isomer $1,101 \mathrm{MHz}, \mathrm{D}_{2} \mathrm{O}$ )

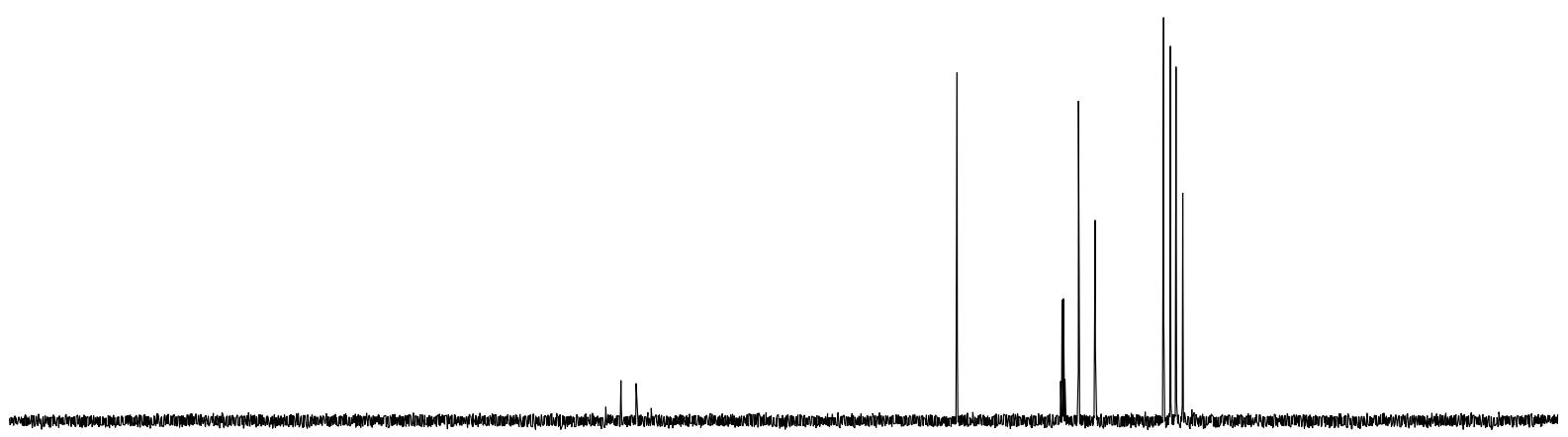

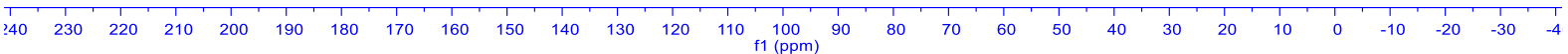




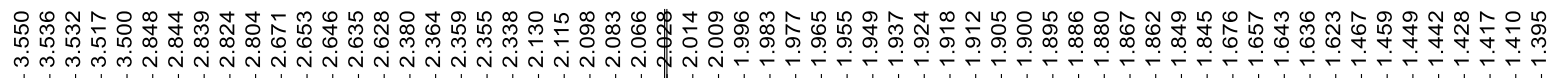

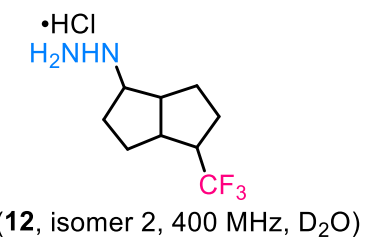

(12, isomer 2, $400 \mathrm{MHz}, \mathrm{D}_{2} \mathrm{O}$ )
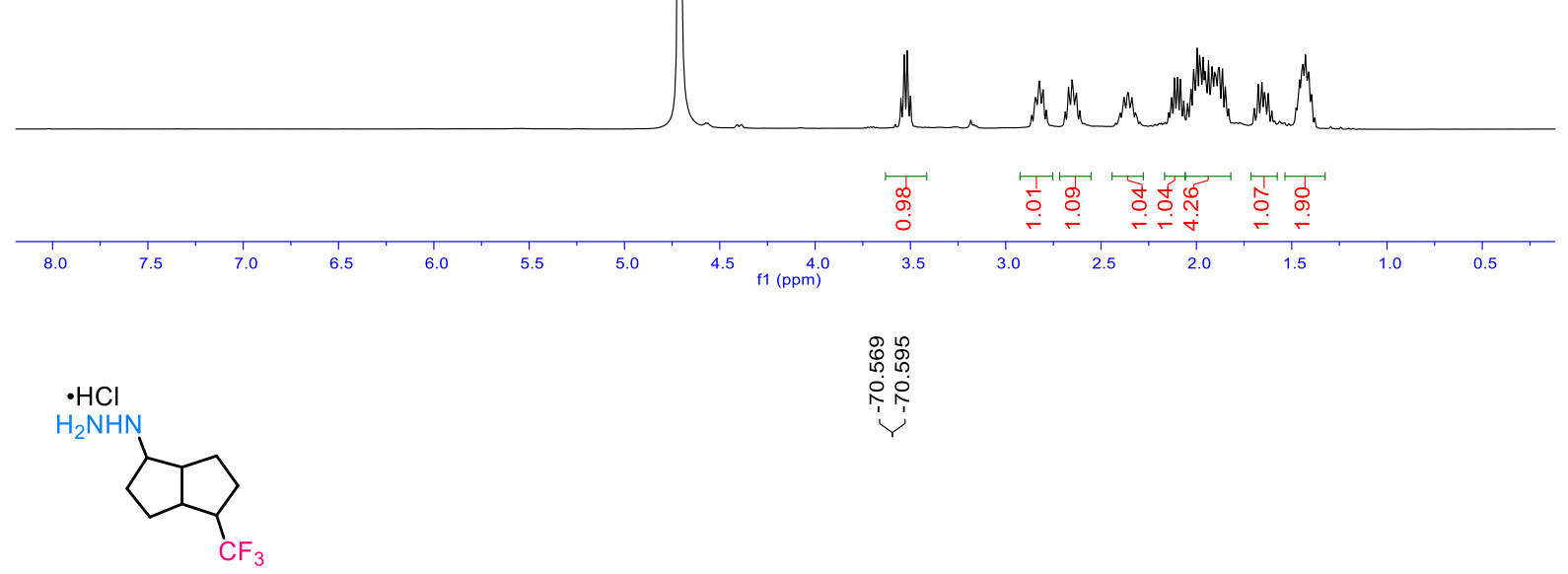

ن

(12, isomer 2, $376 \mathrm{MHz}, \mathrm{D}_{2} \mathrm{O}$ )
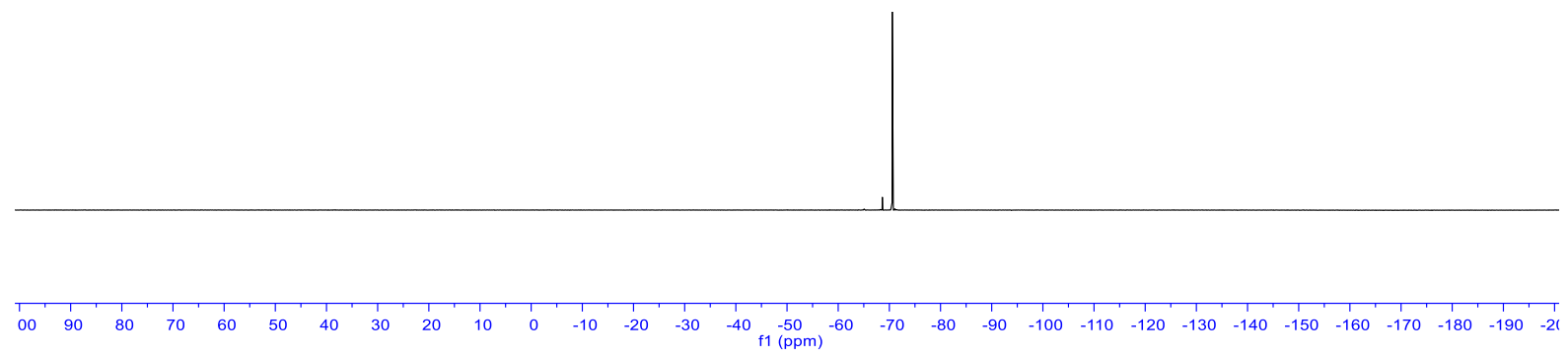

S70 
$\cdot \mathrm{HCl}$

$\mathrm{H}_{2} \mathrm{NHN}$

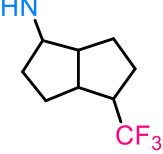

(12, isomer 2, $101 \mathrm{MHz}, \mathrm{D}_{2} \mathrm{O}$ )

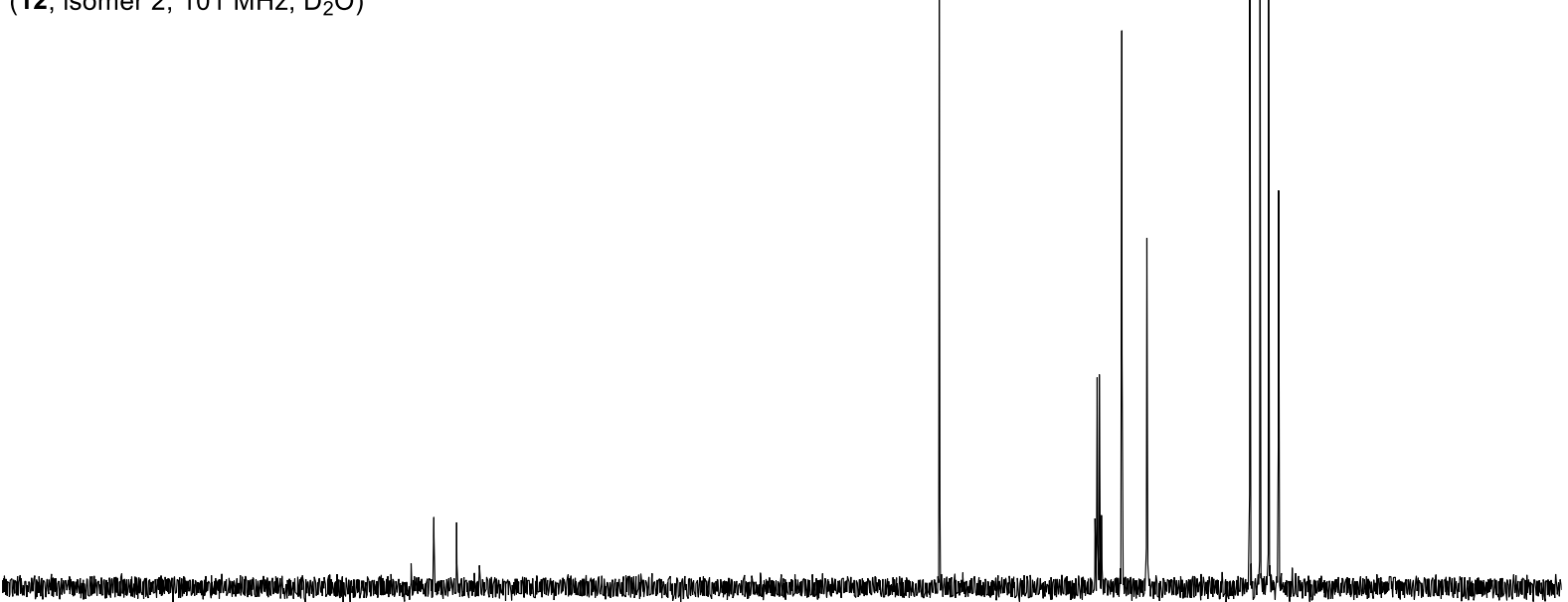

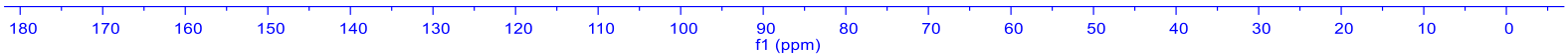

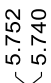

$\cdot \mathrm{HCl}$<smiles>CC(C)(NCl)C1CC=C(CC(F)(F)F)CC1</smiles>

(13, $\left.400 \mathrm{MHz}, \mathrm{MeOH}-d_{4}\right)$

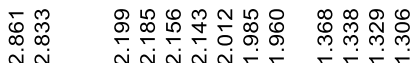

ง

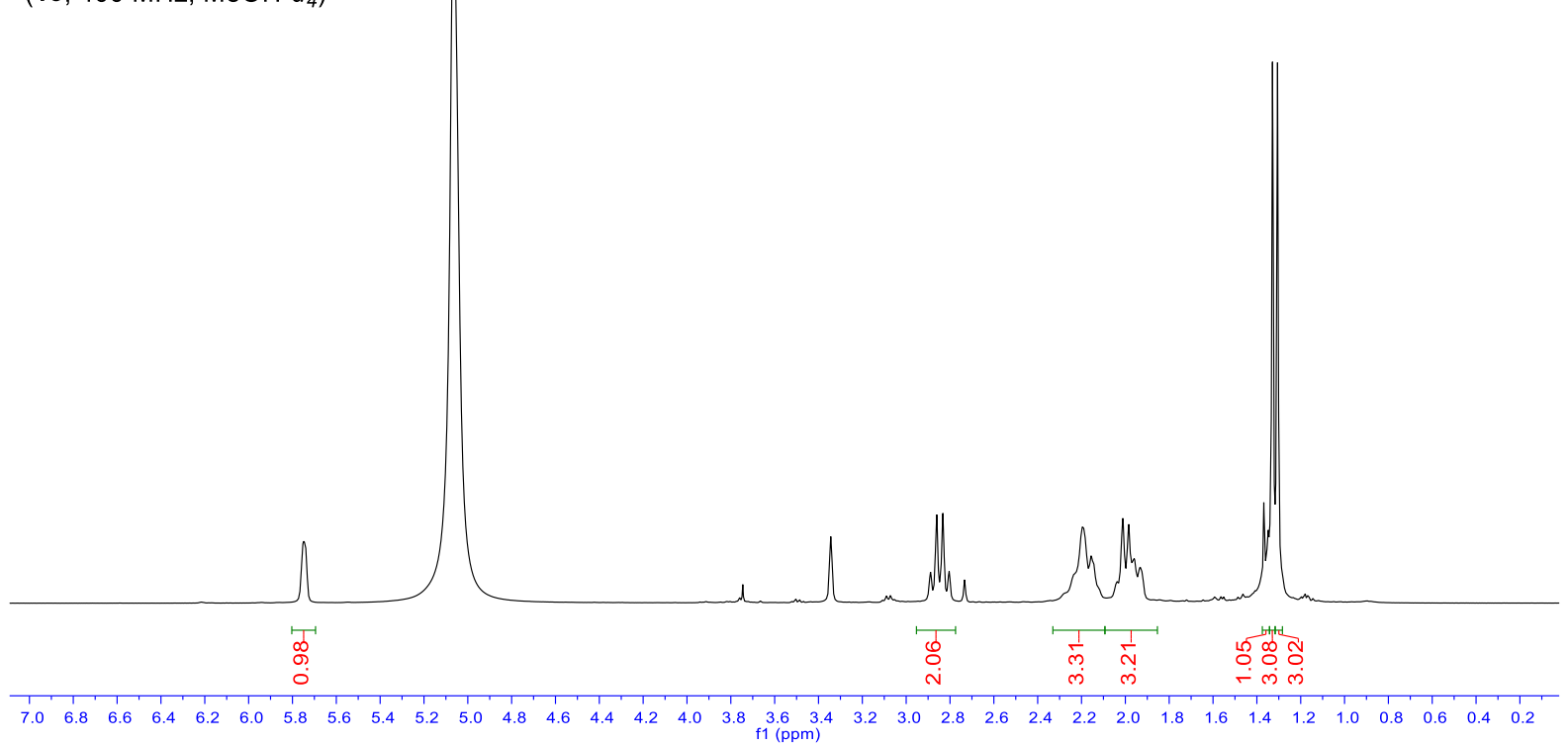




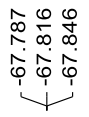
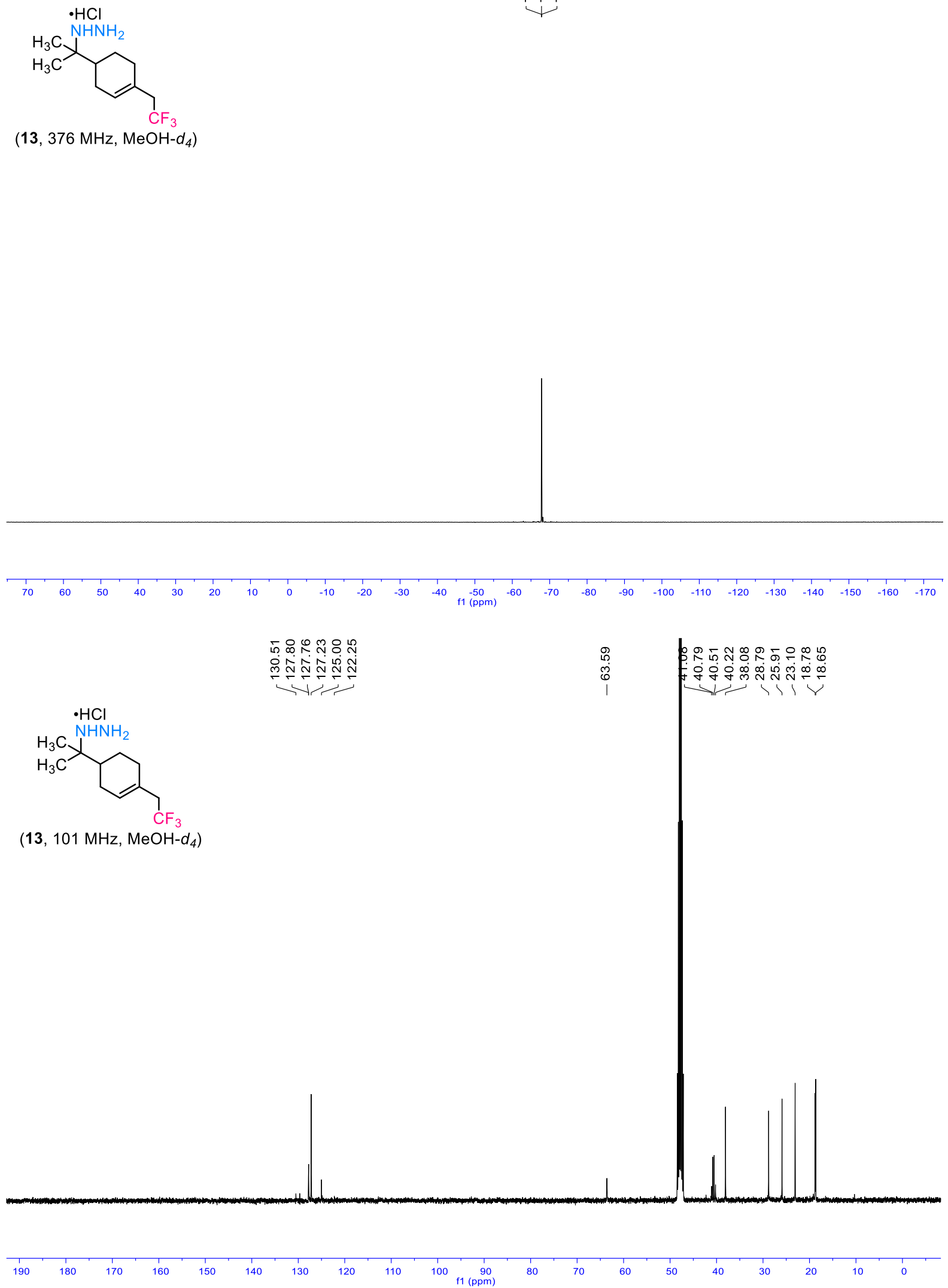

S72 